Portland State University

PDXScholar

Spring 5-30-2018

\title{
Sensing Building Structure Using UWB Radios for Disaster Recovery
}

Jeong Eun Lee

Portland State University

Follow this and additional works at: https://pdxscholar.library.pdx.edu/open_access_etds

Part of the Computer Sciences Commons

Let us know how access to this document benefits you.

\section{Recommended Citation}

Lee, Jeong Eun, "Sensing Building Structure Using UWB Radios for Disaster Recovery" (2018).

Dissertations and Theses. Paper 4388.

https://doi.org/10.15760/etd.6272

This Dissertation is brought to you for free and open access. It has been accepted for inclusion in Dissertations and Theses by an authorized administrator of PDXScholar. Please contact us if we can make this document more accessible: pdxscholar@pdx.edu. 
Sensing Building Structure Using UWB Radios for Disaster Recovery

by

Jeong Eun Lee

A dissertation submitted in partial fulfillment of the requirements for the degree of

Doctor of Philosophy

in

Computer Science

Dissertation Committee:

Suresh Singh, Chair

Wu-chi Feng

Jingke Li

Douglas V. Hall

Portland State University

2018 


\begin{abstract}
This thesis studies the problem of estimating the interior structure of a collapsed building using embedded Ultra-Wideband (UWB) radios as sensors. The two major sensing problems needed to build the mapping system are determining wall type and wall orientation. We develop sensing algorithms that determine (1) loadbearing wall composition, thickness, and location and (2) wall position within the indoor cavity. We use extensive experimentation and measurement to develop those algorithms.
\end{abstract}

In order to identify wall types and locations, our research approach uses Received Signal Strength (RSS) measurement between pairs of UWB radios. We create an extensive database of UWB signal propagation data through various wall types and thicknesses. Once the database is built, fingerprinting algorithms are developed which determine the best match between measurement data and database information. For wall mapping, we use measurement of Time of Arrival (ToA) and Angle of Arrival (AoA) between pairs of radios in the same cavity. Using this data and a novel algorithm, we demonstrate how to determine wall material type, thickness, location, and the topology of the wall.

Our research methodology utilizes experimental measurements to create the database of signal propagation through different wall materials. The work also 
performs measurements to determine wall position in simulated scenarios. We ran the developed algorithms over the measurement data and characterized the error behavior of the solutions.

The experimental test bed uses Time Domain UWB radios with a center frequency of $4.7 \mathrm{GHz}$ and bandwidth of over $3.2 \mathrm{GHz}$. The software was provided by Time Domain as well, including Performance Analysis Tool, Ranging application, and AoA application. For wall type identification, we use the P200 radio. And for wall mapping, we built a special UWB radio with both angle and distance measurement capability using one P200 radio and one P210 radio.

In our experimental design for wall identification, we varied wall type and distance between the radios, while fixing the number of radios, transmit power and the number of antennas per radio. For wall mapping, we varied the locations of reference node sensors and receiver sensors on adjoining and opposite walls, while fixing cavity size, transmit power, and the number of antennas per radio.

As we present in following chapters, our algorithms have very small estimation errors and can precisely identify wall types and wall positions. 


\section{DEDICATION}

In dedication to my husband David and my daughter Eujene for their full support during my study. 


\section{ACKNOWLEDGMENTS}

Firstly, I would like to thank my advisor, professor Suresh Singh of Maseeh College of Engineering \& Computer Science at Portland State University. He provided continued support throughout my Masters \& Ph.D research with great advise, inspiration, and patience. Without him, I wouldn't have completed this long journey.

I would also like to thank the rest of my thesis committee, CS department chair and professor Wuchi Feng, professor Jingke Li, and associate professor Doug Hall, for their guidance and insightful comments.

I also acknowledge Myeonwoo Lim, who was a PSU graduate student and assisted my research laboratory measurement work for an extended period.

I also send my sincere thanks to Asha Keddy, Intel VP at Next Generation and Standards group, who hired me and supported my Ph.D study during full time work.

Last but not the least, my sincere thanks go to my parents and brothers who patiently endured me for this long years until I get my degree far in South Korea. 


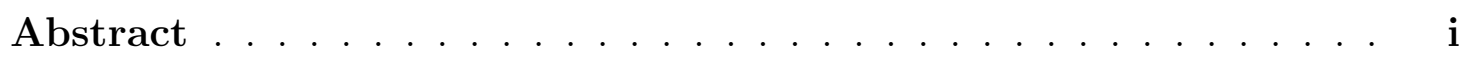

Dedication ............................

Acknowledgments .................. iv

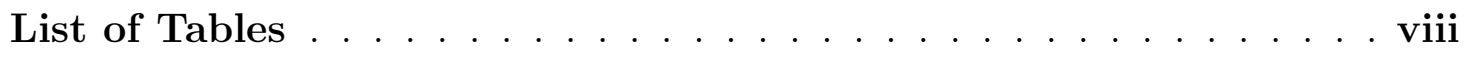

List of Figures $\ldots \ldots \ldots \ldots \ldots \ldots \ldots \ldots \ldots \ldots$ ix

$\begin{array}{lll}\text { Chapter } 1 & \text { Thesis Statement } & 1\end{array}$

Chapter 2 Introduction $\quad 2$

2.1 Research Approach . . . . . . . . . . . . . . . . . . . 6

2.2 Research Methodology . . . . . . . . . . . . . . . . . 9

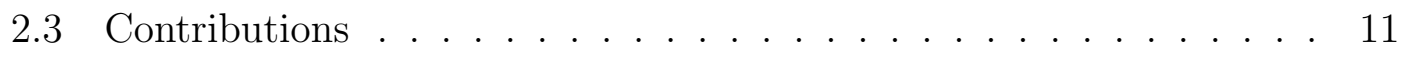

$\begin{array}{lll}\text { Chapter } 3 & \text { Experimental Test Bed } & 13\end{array}$

3.1 UWB Radios . . . . . . . . . . . . . . . . . . . . 13

3.1.1 Transceiver based UWB System . . . . . . . . . . . . 13

3.1 .2 Transmit Power . . . . . . . . . . . . . . . . . . . 16

3.1 .3 Network Setup . . . . . . . . . . . . . . . . . . 16

3.1 .4 SW Setup . . . . . . . . . . . . . . . 17

3.2 Walls and Cavities Radio Measurement Setup . . . . . . . . 17

Chapter 4 Experimental Design for Wall Identification 22

4.1 Laboratory Setup . . . . . . . . . . . . . . . . . 23

4.2 Measurement Database for Wall Identification . . . . . . . . 25

Chapter 5 Results for Wall Type and Thickness Measurement 30

5.1 Freespace Pathloss as Baseline . . . . . . . . . . . . 30

5.2 Pathloss Result for Dry Material . . . . . . . . . . . . 30 
5.2.1 Pathloss Result for Dry Concrete and Other Material . . . . 30

5.2.2 Pathloss Result for Dry Reinforced Concrete and Other Material . . . . . . . . . . . . . . . 32

5.2.3 Dry Concrete of Other Thickness . . . . . . . . . . . 37

5.3 Pathloss Result for Wet Material . . . . . . . . . . . . . 43

5.3.1 Pathloss Result for Wet Concrete and Other Material . . . . 43

5.3.2 Pathloss Result for Wet Reinforced Concrete and Other Material . . . . . . . . . . . . . . . 43

5.3.3 When compared to Dry Material Result . . . . . . . . . . 48

Chapter 6 Wall Type and Thickness Identification 54

6.1 Research Challenge for Wall Type and Thickness Identification . . . 54

6.2 Algorithm for Wall Type and Thickness Identification . . . . . . . . 55

6.3 Wall Type and Thickness Identification Methodology . . . . . . . . 57

6.3.1 Applying Fingerprinting Algorithm . . . . . . . . . . . . 57

6.4 Results for Wall Type and Thickness Identification . . . . . . . . . 60

$\begin{array}{lll}\text { Chapter } 7 & \text { 3D Wall Mapping } & 66\end{array}$

7.1 Algorithm for Radio Position Estimation . . . . . . . . . . . . . . 66

7.1.1 2D Radio Position Estimation Algorithm . . . . . . . . . . . 67

7.1.2 3D Radio Position Estimation Algorithm . . . . . . . . . . . 68

7.2 3D Wall Mapping Methodology . . . . . . . . . . . . . . . 72

7.3 Results for Adjoining Wall Measurement . . . . . . . . . . . . . 76

7.3.1 Results for Adjoining Wall Distance Measurement . . . . . . 76

7.3.2 Results for Adjoining Wall AoA Measurement . . . . . . . . 81

7.3.3 Summary of Adjoining Wall Measurement Results . . . . . . 89

7.4 Results for Opposite Wall Measurement . . . . . . . . . . . . . . . 90

7.4.1 Results for Opposite Wall Distance Measurement . . . . . . 90

7.4.2 Results for Opposite Wall AoA Measurement . . . . . . . . 91

7.4.3 Summary of Opposite Wall Measurement Results . . . . . . 100

7.5 Results for Adjoining Wall Estimation . . . . . . . . . . . . . . . 101

7.6 Results for Opposite Wall Estimation . . . . . . . . . . . . . . . . . 110

7.7 Conclusions for 3D Wall Mapping . . . . . . . . . . . . 115

$\begin{array}{lll}\text { Chapter } 8 & \text { Related Work } & 120\end{array}$

8.1 Material Characterization ................. 120 
8.2 Indoor Positioning . . . . . . . . . . . . . . . . . . . . 122

8.2.1 RSS based Indoor Positioning . . . . . . . . . . . . . . 123

8.2.2 ToA based Indoor Positioning . . . . . . . . . . . . . 123

8.2.3 TDoA based Indoor Positioning . . . . . . . . . . . . . 124

8.2.4 AOA based Indoor Positioning . . . . . . . . . . . . . 124

8.2.5 RSSI based Indoor Positioning . . . . . . . . . . . . . . . 124

8.2.6 Hybrid Schemes for Indoor Positioning . . . . . . . . . . . 125

8.2.7 Challenges and Mitigation for Measurement Error . . . . . . 125

8.3 UWB Radar . . . . . . . . . . . . . . . . . . . . . . 126

$\begin{array}{lll}\text { Chapter } 9 & \text { Conclusions } & 128\end{array}$

$\begin{array}{ll}\text { References } & 130\end{array}$ 


\section{LIST OF TABLES}

6.1 Summary of algorithm performance . . . . . . . . . . . . 61

7.1 Distance measurement result (adjoining wall) . . . . . . . . . . 89

7.2 AoA measurement result (adjoining wall) . . . . . . . . . . . . . 90

7.3 Distance measurement result (opposite wall) . . . . . . . . . . . 100

7.4 AoA measurement result (opposite wall) . . . . . . . . . . . . 101

7.5 Target node estimation result (adjoining wall) . . . . . . . . . . 106

7.6 Target node estimation result (opposite wall) . . . . . . . . . . 110 


\section{LIST OF FIGURES}

2.1 Interior structure of a collapsed building . . . . . . . . . . . . . . . 3

2.2 An Example of UWB signal in frequency domain (left) and time domain (right) $[77] \ldots \ldots \ldots 6$

3.1 PulseOn P200 Radio (left) and PulseOn P210 Radio (Right) . . . . 14

3.2 The basic features of an UWB Impulse Radio. The top image shows the Fourier conjugates, the sinc pulse and the square function. The bottom image shows the time series for the Hitachi UWB IR [90] . . 15

3.3 Network setup between a PC and the UWB radios . . . . . . . . . . 16

3.4 Performance Analysis Tool (PAT) application . . . . . . . . . . . . 18

3.5 The waveform scan window of Performance Analysis Tool . . . . . . 18

3.6 Range demonstration application . . . . . . . . . . . . . . . . . . 19

3.7 Angle of Arrival (AoA) demonstration application . . . . . . . . . . 19

3.8 Radio setup for sensor 1 and sensor 2 for wall mapping . . . . . . . 20

3.9 Illustration of AoA calculation method . . . . . . . . . . . . . . 21

4.1 Laboratory setup for wall type and thickness measurement . . . . . 24

4.2 RescueNet website for measurement database access . . . . . . . . . 27

4.3 Sample database query result . . . . . . . . . . . . . . . . . 28

4.4 Example database analysis on RescueNet website . . . . . . . . . . 29

5.1 Freespace pathloss ................... 31

$5.266^{\prime \prime}$ dry concrete pathloss . . . . . . . . . . . . . . . . . 32

$5.366^{\prime \prime}$ dry concrete with plywood . . . . . . . . . . . . . . 33

5.4 Dry concrete with drywall . . . . . . . . . . . . . . . . 34

5.5 Dry concrete with plywood and drywall . . . . . . . . . . . . 35

5.6 Dry concrete with other material . . . . . . . . . . . . . 36

5.7 Dry reinforced concrete pathloss . . . . . . . . . . . . . . 37

5.8 Dry reinforced concrete with plywood . . . . . . . . . . . . 38

5.9 Dry reinforced concrete with drywall . . . . . . . . . . . . . . . . 39 
5.10 Dry reinforced concrete with plywood and drywall . . . . . . . . . . 40

5.11 Dry reinforced concrete with other material . . . . . . . . . . . 41

$5.124 .5^{\prime \prime}$ dry concrete with other materials . . . . . . . . . . . . . . 42

$5.138^{\prime \prime}$ dry concrete with other materials . . . . . . . . . . . . . . 42

5.14 Wet concrete pathloss . . . . . . . . . . . . . . . 44

5.15 Wet concrete with wet plywood . . . . . . . . . . . . 45

5.16 Wet concrete with dry drywall . . . . . . . . . . . . . . . 46

5.17 Wet concrete with wet plywood and dry drywall . . . . . . . . . . . 47

5.18 Wet concrete with other material . . . . . . . . . . . . . . . 48

5.19 Wet reinforced concrete pathloss . . . . . . . . . . . . . . . 49

5.20 Wet reinforced concrete with wet plywood . . . . . . . . . . 50

5.21 Wet reinforced concrete with dry drywall . . . . . . . . . . . . 51

5.22 Wet reinforced concrete with wet plywood and dry drywall . . . . . 52

5.23 Wet reinforced concrete with other material . . . . . . . . . . . 52

5.24 Dry versus wet material . . . . . . . . . . . . . 53

6.1 Radio locationing and material sensing example . . . . . . . . . 55

6.2 Illustration of the algorithm . . . . . . . . . . . . . . 58

6.3 Pathloss for a subset of dry material . . . . . . . . . . . . . 59

6.4 Error in distance estimate for freespace . . . . . . . . . . . . . 62

6.5 Error in distance estimate for plywood . . . . . . . . . . . . . 63

6.6 Error in distance estimate for concrete . . . . . . . . . . . . . 64

6.7 Error in distance estimate for reinforced concrete . . . . . . . . 65

7.1 Position of target node in 2D space . . . . . . . . . . . . . 67

7.2 Position of target node in 3D space . . . . . . . . . . . . . . . 69

7.3 3D wall measurement experimental setup . . . . . . . . . . . . . 73

7.4 Adjoining wall measurement setup . . . . . . . . . . . . . . 74

7.5 Opposite wall measurement setup . . . . . . . . . . . . . . . . 75

7.6 Adjoining wall distance estimation error per sensor 1 location . . . 78

7.7 Adjoining wall distance estimation error per sensor 2 location on each row . . . . . . . . . . . . . . . . . . . 79

7.8 Adjoining wall distance estimation error per sensor 1 and sensor 2 location . . . . . . . . . . . . . . 80

7.9 Adjoining wall angle estimation error per sensor 1 location (relative error $\ldots \ldots \ldots \ldots$. . . . . . . . . . . . . 83 
7.10 Adjoining wall angle estimation error per sensor 1 location (absolute

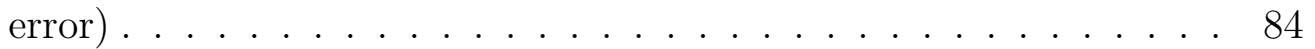

7.11 Adjoining wall angle estimation error per sensor 2 location on each row (relative error) . . . . . . . . . . . . . . 85

7.12 Adjoining wall angle estimation error per sensor 2 location on each row (absolute error) . . . . . . . . . . . . 86

7.13 Adjoining wall angle estimation error per sensor 1 and sensor 2 location (relative error) . . . . . . . . . . . 87

7.14 Adjoining wall angle estimation error per sensor 1 and sensor 2 location (absolute error) . . . . . . . . . . . . . . 88

7.15 Opposite wall distance estimation error per sensor 1 location . . . . 92

7.16 Opposite wall distance estimation error per sensor 2 location on each row . . . . . . . . . . . . . . . . . . 93

7.17 Opposite wall distance estimation error per sensor 1 and sensor 2 location . . . . . . . . . . . . . . . . . 9 94

7.18 Opposite wall angle estimation error per sensor 1 location . . . . . . 96

7.19 Opposite wall angle estimation error per sensor 2 on each row . . . 97

7.20 Opposite wall angle estimation error per sensor 1 and sensor 2 location (absolute error) . . . . . . . . . . . . . . . . . . 98

7.21 Opposite wall angle estimation error per sensor 1 and sensor 2 location (relative error) . . . . . . . . . . . . . . . . . 99

7.22 Estimated target positions with connected lines from actual target positions (Adjoining wall) . . . . . . . . . . . . . . 102

7.23 Estimated target positions that exclude estimations with imaginary numbers (Adjoining wall) . . . . . . . . . . . . 103

7.24 Estimated target positions with excluded estimations with imaginary numbers and from straight line reference nodes (Adjoining wall) 104

7.25 CDF of target node position estimation MSE (adjoining wall) . . . 105

7.26 Reference node combinations with minimum target estimation error (Adjoining wall) . . . . . . . . . . . . . . 107

7.27 Normalized relative target position estimation error for adjoining wall (normalized to inter-target distance of $30 \mathrm{~cm}$ ) . . . . . . . . 108

7.28 Minimum relative target position estimation error for adjoining wall 109

7.29 Estimated target positions with connected lines from actual target positions (Opposite wall) . . . . . . . . . . . . . 111 
7.30 Estimated target positions with excluded estimations with imaginary numbers (Opposite wall) . . . . . . . . . . . . . . 112

7.31 Estimated target positions with excluded estimations with imaginary numbers and from straight line reference nodes (Opposite wall) 113

7.32 CDF of target node position estimation MSE (opposite wall) . . . . 114

7.33 Reference node combinations with minimum target estimation error (opposite wall) . . . . . . . . . . . . . . . 116

7.34 Normalized relative target position estimation error from each reference node combination (opposite wall) . . . . . . . . 117

7.35 Minimum relative target position estimation error (opposite wall) . 118 
Chapter 1

\section{THESIS STATEMENT}

Can we use properties of radio signal propagation to map the interior structure of buildings and construction material type? What are the limitations of using this technique in real life? 
Chapter 2

\section{INTRODUCTION}

When a building collapses following earthquakes, hurricanes, tsunami, or other causes, trapped survivors need to be rescued as quickly and safely as possible. Time is of great importance during search and rescue operations in the aftermath of a disaster. The first 24 hours after a disaster has been called the "Golden Day", the period during which injured or trapped victims have an 80 percent chance of survival [25].

Rescuing trapped survivors after building collapse is a very slow and difficult effort due to unstable and unknown structure of the damaged buildings and lack of real-time assistance tools to aid damage assessment and rescue planning. Figure 2.1 shows the interior structure of such collapses. We see that cavities are formed by structural material and survivors are trapped in those cavities. Rescuers need to dig through the rubble cautiously in order to prevent a secondary collapse, which can cause further loss of lives of both rescuers and victims. Thus, knowledge of the interior structure of the collapse, specifically the wall composition and the shape of the cavities, is essential in order to locate and rescue potential survivors in a timely manner.

Traditional rescue tools to assist in locating survivors and surveying structural topology consists of heartbeat sensors [69][53][47], acoustic sensors, search cameras, or small robots [66][64][36] that are inserted into small openings though the rubble 


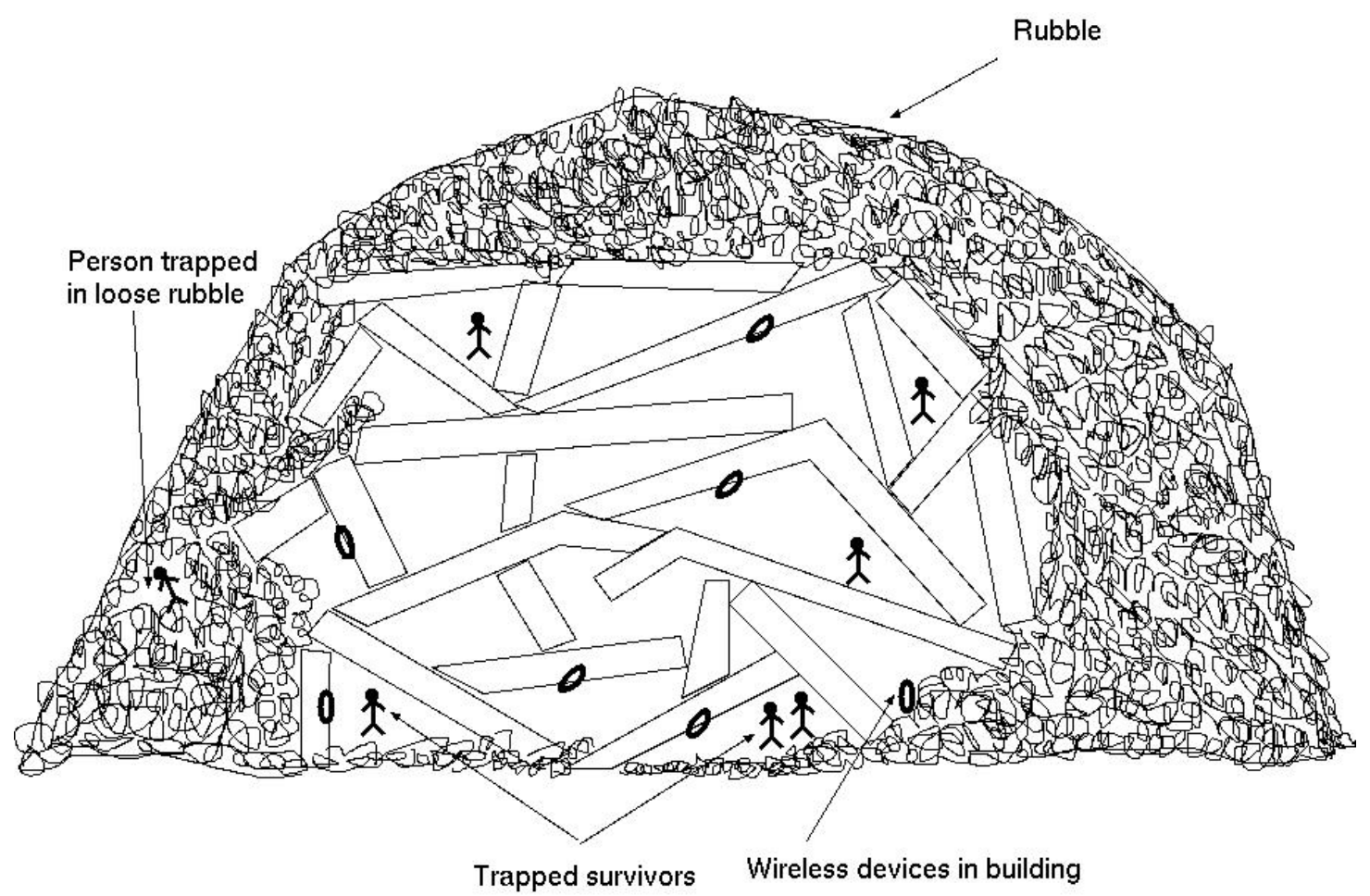

Figure 2.1. Interior structure of a collapsed building

pile, and ground penetrating radar (GPR) [49][62][67][81][94]. However, most of these tools have their own limitations in that they can survey only the top part of the rubble pile and cannot examine the structures and survivors buried deep in the collapse. The rescue operation can be expedited if a map of the interior structure of the collapsed building is available, which identifies the location of the walls and the shape of the cavities.

The overall disaster rescue scenarios will involve the following steps:

1. Battery-operated sensors are deployed a priori on interior walls of the building that turn on automatically in the event of building collapse, triggered by their vertical alignment change. 
2. These sensors use Ultra-Wideband (UWB) radio to determine the loadbearing wall types and locations. Sensors coordinate sending and receiving and begin sensing the material as well as location of others by broadcasting signals and receiving signals to and from neighbor sensors.

3. Sensors form a multi-hop ad hoc network in which each sensor participates in routing (e.g. flooding, SPIN, Rumor routing, energy aware routing [43][44][52] $[10][104][80]$ ) in order to relay the local sensing information (e.g. wall type, and sensor location) to rescuers outside.

4. The external node with computing power will fuse the received sensing data for analysis to create a cavity map of the interior structure.

In step 1 above, in order to turn on sensors, accelerometers [95][48][82] can be utilized which can detect the change of velocity and angle of the moving objects. The event that causes the destructive structural change in the building is a rare event, thus sensors are expected to have a long lifetime. The long lifetime can be achieved by combining multiple power saving techniques such as forced excitation-based sensors that operate on an extremely low-duty cycle, low-power listening, hierarchy based asynchronous wakeup, processing, and radio transmission [27][17][80][5][6].

In step 2 and 3, sender-receiver synchronization can be done with a conventional approach of handshaking between a pair of nodes, TPSN (Timing-sync Protocol for Sensor Networks), or post-facto synchronization [32][29]. However, in our research, clock synchronization between a sender and a receiver is not required because the sender uses time of arrival for the returned signal from the receiver. In order to minimize the complexity of sensors in step 3, sensors are not required to track its absolute location. Instead, the powerful external node is responsible for deriving 
global location of sensors from the forwarded relative location information [12][14]. The radios cooperatively form an ad hoc network from the inside out to forward all the collected data to rescuers who are on the outside of the rubble. This process is a well-studied one in general mobile ad hoc networking with many available solutions. Sensors can coordinate transmission and reception of data using various MAC protocols such as carrier sense multiple access [30][102]. There is a limitation on this approach in that there can be areas where sensing information cannot be collected due to no sensors nearby. However, a partial map created by the incomplete information will be also useful.

In order to build the complete rescue mapping system, there are major sensing problems to solve, such as (1) detecting people via heartbeats or respiration rate, (2) identifying cavity shapes, (3) determining orientation of the supporting walls, and (4) determining wall composition, thickness, and location with respect to sensors.

The goal of this research is to develop sensing algorithms for a subset of the whole sensing problem using experimentation and measurement. In this research the focus of the work is on step 2 noted above. Specifically, this research focuses on two areas:

- Determining wall composition, thickness, and location (with respect to sensors)

- Determining radio/wall positions in indoor cavities

Once sensing is done, a data fusion step will combine measured data to complete constructing the interior structure map. However, data fusion to build a complete map using other types of sensing data is out of scope of this research. 


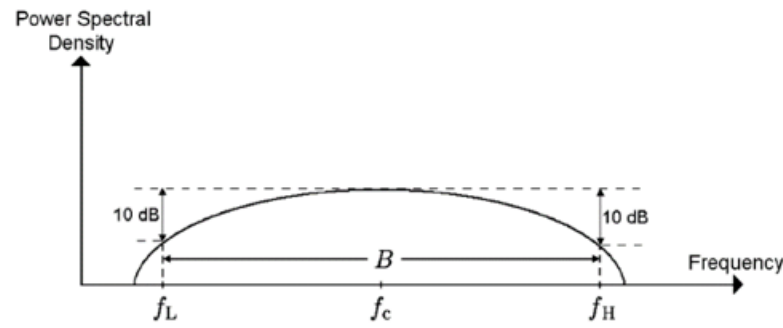

(a)

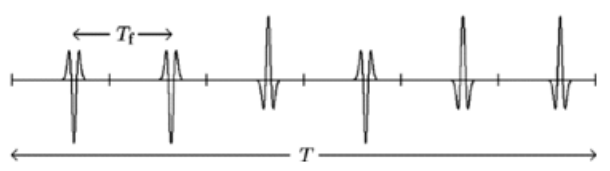

(b)

Figure 2.2. An Example of UWB signal in frequency domain (left) and time domain (right) [77]

\subsection{RESEARCH APPROACH}

Ultra-wideband (UWB) wireless technology has been the subject of extensive research in the area of wireless sensing in recent years. With the advent of 5G wireless technology that envisions billions of smart and connected devices, the importance of high resolution positioning systems in hostile signal propagation environment is critical [3]. Due to its unique capabilities as explained further below, UWB satisfies the requirements of precise estimation of position, wall orientation, and building structure. We aim to utilize embedded UWB radios as wireless sensors with a goal to aid disaster recovery.

UWB is a radio technology that operates on a spectrum of $3.1 \mathrm{GHz}$ to $10.6 \mathrm{GHz}$. As the name implies, UWB occupies significantly large bandwidth of the spectrum of the order of many GHz. FCC defines UWB signals as having an absolute bandwidth of at least $500 \mathrm{MHz}$ or a fractional (relative) bandwidth of larger than $20 \%$ [18]. As shown in figure 2.2 (a), the absolute bandwidth, $B$, is obtained as the difference between the upper frequency $f_{H}$ and the lower frequency $f_{L}$ (i.e., $f_{B}=f_{H}-f_{L}$ ), whereas the fractional bandwidth, $f_{F B}$, is calculated as a ratio of bandwidth and center frequency, $f_{c}$, when $f_{c}=\left(f_{H}+f_{L}\right) / 2$, i.e.: 


$$
\begin{aligned}
f_{F B} & =\frac{f_{B}}{f_{C}} \\
& =\frac{2\left(f_{H}-f_{L}\right)}{f_{H}+f_{L}}
\end{aligned}
$$

The pulse duration of an electromagnetic signal $T_{p}$ and its bandwidth $B$ satisfies an inverse relation given by [65]:

$$
B \approx \frac{1}{T_{p}} .
$$

Thus, the wider the bandwidth, the shorter the pulse duration, and the narrower the bandwidth, the longer the pulse duration. Due to the inverse relation of the bandwidth and the pulse duration, UWB radios generate very short pulses to generate ultra-wide bandwidth signals (figure 2.2 (b)), resulting in a high time resolution of UWB signals. The high time precision of the UWB signal can be exploited for accurate measurement of distance and time [77]. Also, its property to use short pulses makes signal reflections barely overlap with the primary signal, and thus helps UWB be resistant to multipath [96].

Another property of UWB is that it has improved signal penetration to obstacles due to the presence of lower frequency components as well as high frequency components [35][8]. This is because different radio frequencies have different physical properties as they propagate through media. As shown in equation 2.3, the wavelengths of lower frequencies are longer than that of higher ones, allowing the lower frequencies propagate farther than higher frequencies:

$$
f=\frac{v}{\lambda},
$$


where $v$ is the phase velocity and $\lambda$ is the wavelength of the wave. Radio signals with longer wavelength tend to hug the edge of obstacles and be less susceptible to atoms and molecules, whereas the higher frequency signals with shorter wavelength tend to be blocked by them. These unique properties together with low energy consumption have made UWB widely accepted for developing applications in the areas of wireless sensing or precise positioning.

In our research, we assume that every wall of a building has an embedded UWB radio. Upon collapse, these embedded UWB radios will be tasked to perform the two important sensing tasks of wall identification and cavity mapping. The following approaches were taken to achieve each task.

1. Wall identification: In order to identify wall material properties such as type, thickness, and location with respect to the radios, we used Received Signal Strength (RSS) measurements between pairs of UWB radios separated by varying types of walls in cavities. This approach is feasible since building construction walls come in only a few types (e.g. reinforced concrete, concrete, and ply-wood) and standard thicknesses. For example, $8^{\prime \prime}$ reinforced concrete is typically used as a load bearing material in building construction. This approach requires creating an extensive database of signal propagation measurement through various wall types and thicknesses. Once the database of signal propagation measurements is built, fingerprinting algorithms [7][39][40][50][51] are developed which determine the best match between in-field measurement and information derived from the database in order to determine wall material type, thickness, and location in an actual collapse. 
2. Wall mapping: Radio locationing and wall mapping will identify the location of the radios in the cavity in which a radio finds itself after a collapse. The approach we study uses measurement of Time of Arrival (ToA) and Angle of Arrival (AoA) between pairs of radios within the same cavity. Assuming that each embedded radio knows its original placement (i.e., the wall it is embedded in), we developed algorithms that use the ToA based distance information to construct the topology of the wall [35]. The collective positioning information from multiple sets of radios provides a view of the wall.

\subsection{RESEARCH METHODOLOGY}

The methodology for this research utilizes extensive measurements for creating the database of signal propagation through different materials. The work simultaneously performs measurements to study the capability of these radios to determine position information in indoor cavities. Finally, algorithms were developed that fuse the measurement data for wall identification and also fuse positioning components to estimate the location of the radios and view of the wall on which those radios reside in a collapsed cavity.

The experimental measurements were carried out utilizing Time Domain UWB radios [90][91] with walls and cavities built in the Civil Engineering department. We performed "real life" measurements in simulated scenarios and ran our developed algorithms on the collected data in order to test the developed algorithms. We characterized the error behavior of the developed solution.

We summarize below the experiments that were performed to develop and verify wall identification algorithms: 
- Measure signal propagation through various types and thicknesses of building materials, and characterize the path loss characteristics of the materials. We created a database with those measurements, which was made publicly accessible [55]. RSS was measured between two radios through a wall that separates the two radios in non-line-of-sight (NLOS) environment. Sample debris walls were constructed in a lab.

For radio locationing and wall mapping, measurement data was collected between a pair of UWB sensors in the same room that simulates a cavity. Measurements were done at various locations on the walls as well as various orientations of walls to understand the signal propagation characteristics based on the various configurations of radio and wall positions. Then, measurement data was analyzed to understand the error characteristics of in-room propagation and the accuracy of individual ranging. Once measurement data was analyzed, algorithms for estimating the radio location and wall position was developed. For radio locationing and wall mapping, ToA and AoA data was collected.

We specify below the experiments for radio locationing and wall mapping algorithm development:

- Perform the AoA and ToA measurements when the radios are in the same cavity for estimation of wall orientation and radio location. Both AoA and ToA measurements were performed in line-of-sight environment.

The various configurations of a cavity was simulated in a laboratory environment, which enabled us to study the core problems in a controlled environment and to change the settings and parameters to our need. The developed algorithms - radio 
locationing, material identification, and cavity mapping - can be refined further in future works based on the experiments performed in the laboratory setting.

\subsection{CONTRIBUTIONS}

This research provides direct human benefit in disaster rescue missions by developing a useful tool for mapping collapsed structures. Technical contributions include:

- Proof of concept of using embedded UWB radios as sensors to aid in mapping fallen buildings from the inside.

- This research generated a, first of its kind, publicly available database of UWB signal propagation measurements through various thicknesses and composition of building materials in various configurations. This will be useful in understanding non-line-of-sight (NLOS) signal propagation characteristics and modeling for UWB communications. In addition, this study will provide a comprehensive understanding of using UWB for sensing.

- A detailed measurement study of UWB-based ToA and AoA indoor positioning in complex multi-path environments. Measurement-based study of error in estimation of distances and angles for locationing.

- Data fusion algorithms for creating topological estimates of radio and wall locations in indoor cavities using the UWB based material sensing and positioning capabilities. Analysis of error in such estimation and characterization of where these techniques can be used and where we may need other techniques. 
The remainder of this thesis is organized as follows. In the next chapter we discuss the experimental test-bed. Chapter 4 explains the experimental design for wall identification while chapter 5 discusses the measurement results. Chapter 6 summarizes the results for wall identification, and chapter 7 discusses wall mapping algorithm and results. Chapter 8 summarizes related work on material sensing and indoor positioning. Finally, chapter 9 concludes the thesis. 
Chapter 3

EXPERIMENTAL TEST BED

\subsection{UWB RADIOS}

The experimental measurements were carried out utilizing Time Domain UWB radios, the PulseOn P200 [90] and the P210 [91] as shown in Figure 3.1. These radios transmit very short pulses, which gives us high accuracy in time or distance estimation. The pulses are centered at $4.7 \mathrm{GHz}$ and have a UWB bandwidth of 3.2 $\mathrm{GHz}$, giving us a time accuracy of the order of a nanosecond. This translates to a distance accuracy of better than $10 \mathrm{~cm}$. The pulses are generated every $104.2 \mathrm{~ns}$ (=1 frame). The antenna module is an omni-directional planar dipole.

\subsubsection{Transceiver based UWB System}

There are two types of UWB systems, i.e., UWB transceiver system and UWB radar system. The UWB system used in our experiment is a transceiver based system. Transceiver based UWB system has a combined transmitter and receiver that actively generates and transmits a return signal on receipt of an incoming signal while radar based UWB system passively reflects the incoming signal [28]. Transceiver based UWB system has potential for a higher Signal to Noise Ratio (SNR) than passive UWB radar system. The amplitude of the return signal will

have a range, $\mathrm{r}$, dependence of $r^{-2}$, as opposed to $r^{-4}$ for a radar system, and it will be independent of the reflectivity of the illuminated surface. 

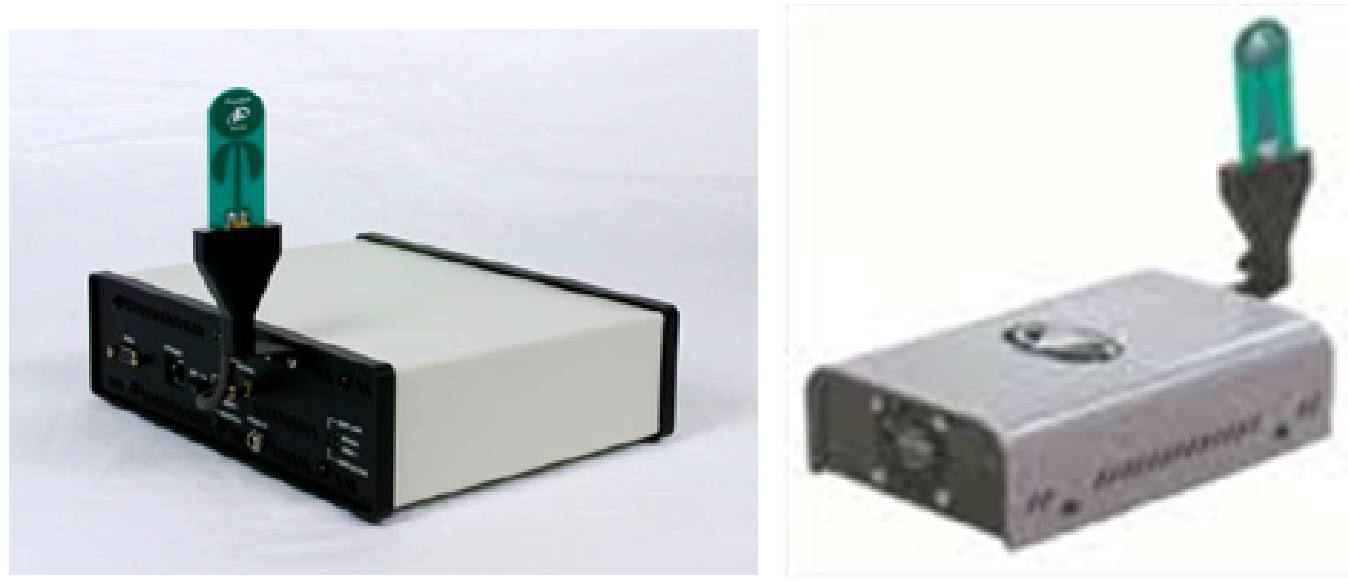

Figure 3.1. PulseOn P200 Radio (left) and PulseOn P210 Radio (Right)

UWB transceiver system has benefit over UWB radar in that the position of the UWB transceiver can be accurately determined, thus the precise location of the responder radio can be obtained. Furthermore, the amplitude and location of the responder radio can be determined independent of the conditions, orientation, and geometry of the object. On the other hand, in a UWB radar system, the amplitude and location of the object will be significantly dependent on the conditions, orientation, and geometry of the object.

The UWB transceiver system is often referred to as UWB Impulse Radio (UWB IR) becuase it generates a time series of very short impulse like pulses in the time domain, with the pulse width of the order of nanoseconds and the bandwidth of the order of $\mathrm{GHz}$ as shown in Figure 3.2. Different from conventional radios, UWB IRs do not generate a modulated carrier wave. $1 / B$ seconds in the time domain in the figure corresponds to a square pulse with a bandwidth of approximately $B \mathrm{~Hz}$ in the frequency domain. Shorter pulses correspond to broader bandwidths. The lower image shows an example of the time series for the Hitachi UWB IR [90]. 


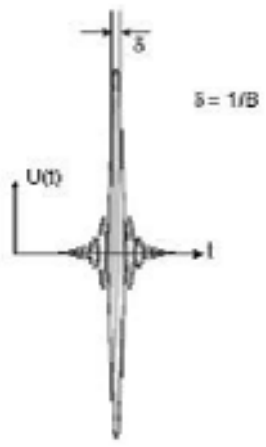

Time Domain

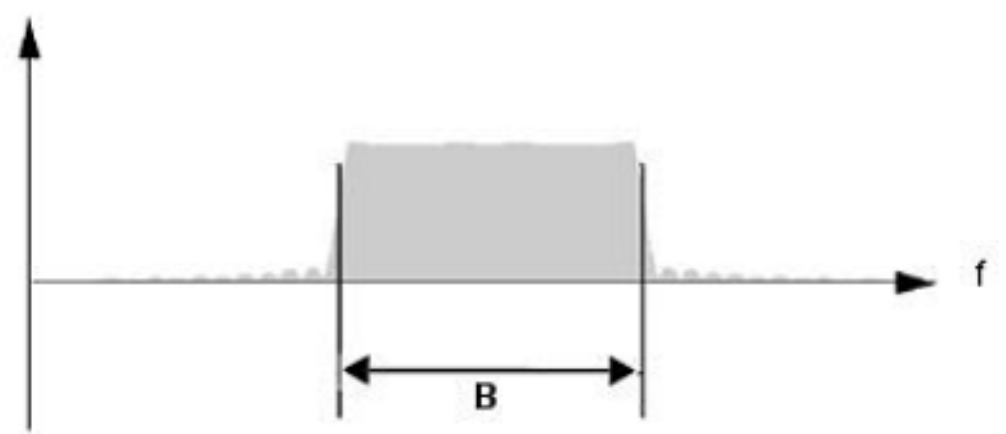

Frequency Domain

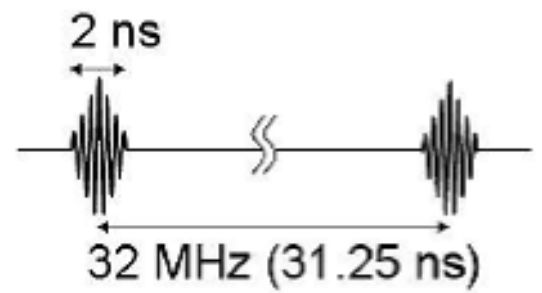

Figure 3.2. The basic features of an UWB Impulse Radio. The top image shows the Fourier conjugates, the sinc pulse and the square function. The bottom image shows the time series for the Hitachi UWB IR [90] 


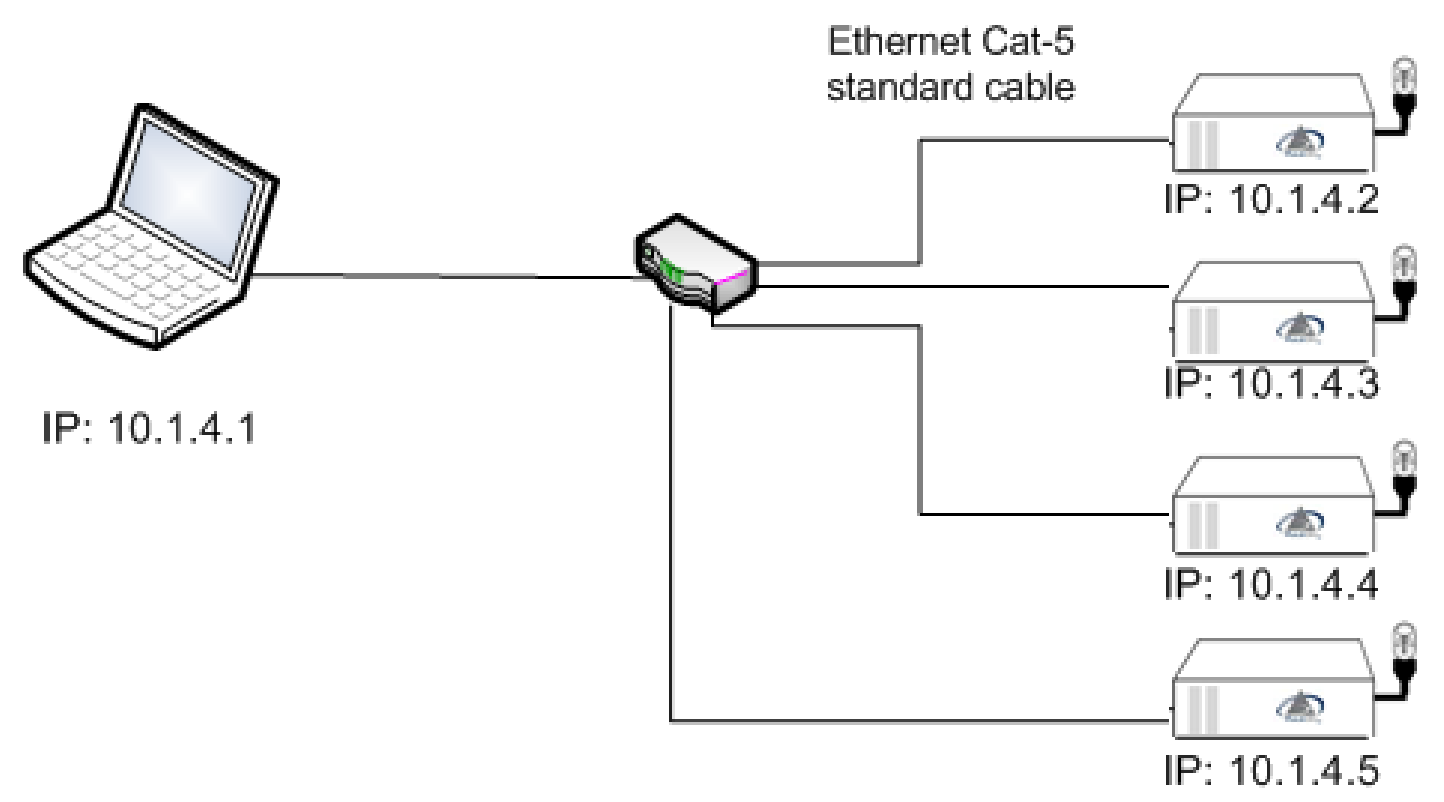

Figure 3.3. Network setup between a PC and the UWB radios

\subsubsection{Transmit Power}

The FCC power spectral density emission limit for UWB transmitters is -41.3 $\mathrm{dBm} / \mathrm{MHz}$. This has been the basis of power limits of UWB systems. The Time Domain PulseOn P200 and P210 radios were designed to be compliant to FCC regulations, so the average transmit power is 50 microwatts $(=-13 \mathrm{dBm})$.

\subsubsection{Network Setup}

The radios were controlled by a laptop for loading embedded software on the radio, configuring measurement parameters, and fetching measurement results from radios. The laptop and the radios were connected through a router using standard Ethernet cables as shown in Figure 3.3 [91]. The PC and radios were configured with different static IP addresses. Once connected, the PC to radio interface was tested by establishing the Ethernet connection using telnet. 


\subsubsection{SW Setup}

For our experimental measurement, the SW (Software) application provided by Time Domain was used. The SW applications configure test parameters, start/stop the measurement and fetch measurement results from the radio. Figure 3.4, Figure 3.5, Figure 3.6, and Figure 3.7 show the screen capture of applications. Each kind of applications run as a pair on the controller laptop, one as a Tx (transmitter) mode and the other as a $\mathrm{Rx}$ (receiver) mode to control a transmitter radio and a receiver radio each.

- Performance Analysis Tool (Figure 3.4) [90] enables scanning UWB waveforms (Figure 3.5) and provides amplitude values. It is used to obtain RSS measurements.

- Range Demonstration Application (Figure 3.6) [92] is used for distance ranging.

- AoA Demonstration Application (Figure 3.7) [89] displays the AoA with the white line. The zero degree display is shown in Figure 3.7.

\subsection{WALLS AND CAVITIES RADIO MEASUREMENT SETUP}

For wall identification experimentation, two P200 radios were used for RSS (Received Signal Strength) measurement. For wall mapping research, in order to build a sensor with both angle and distance measurement capability, one P200 radio and one P210 radio were put together as shown in Figure 3.8. The former is an AoA sensor and the latter is a distance ranging sensor. Sensor 1 estimates the AoA and distance of sensor 2 . 


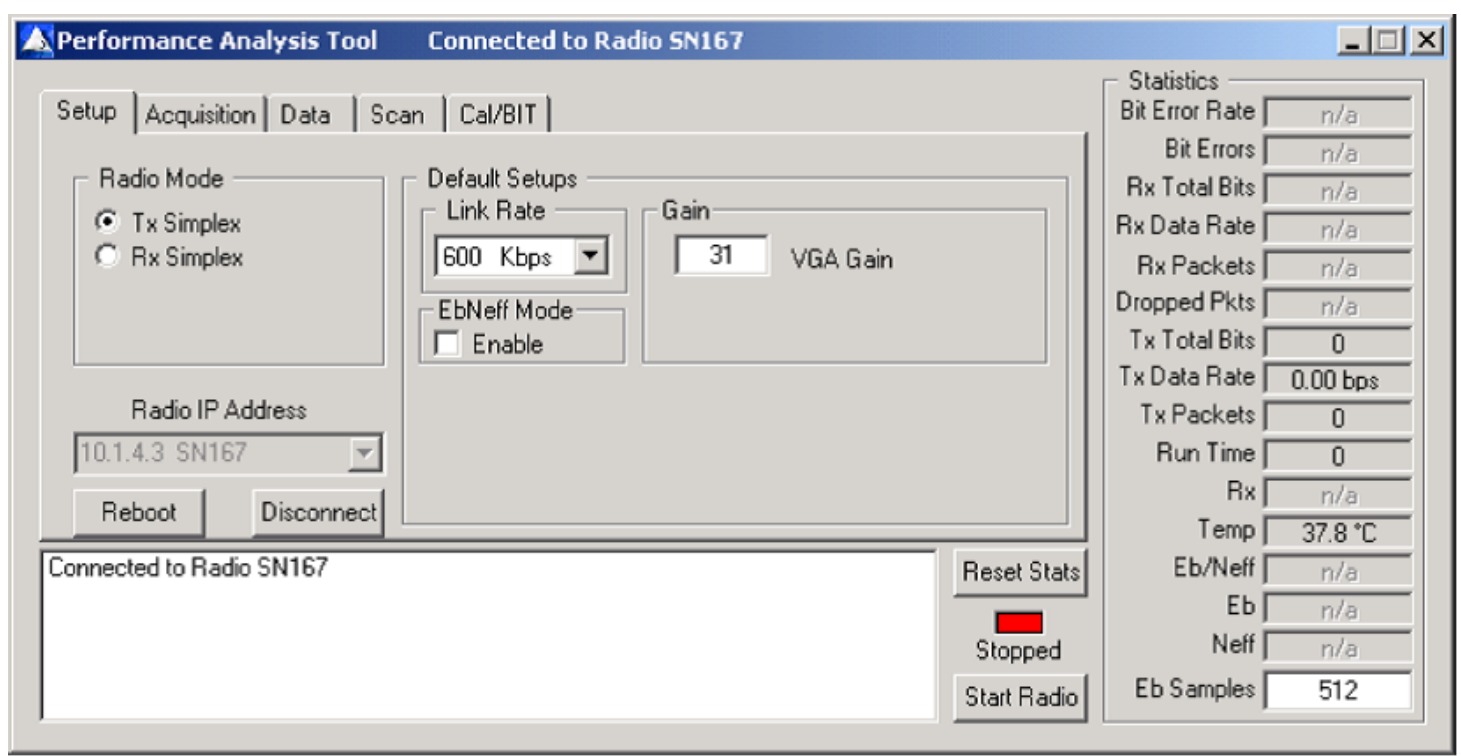

Figure 3.4. Performance Analysis Tool (PAT) application

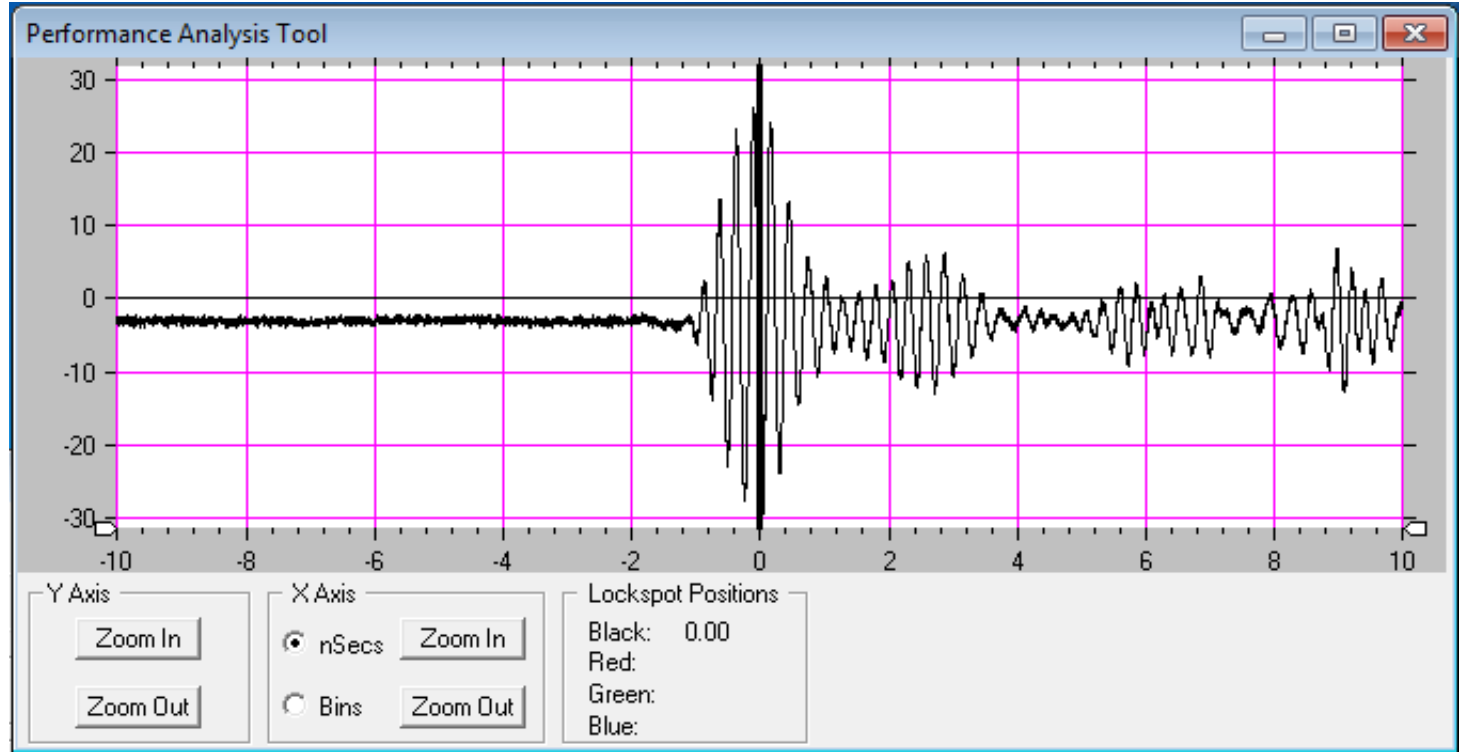

Figure 3.5. The waveform scan window of Performance Analysis Tool 


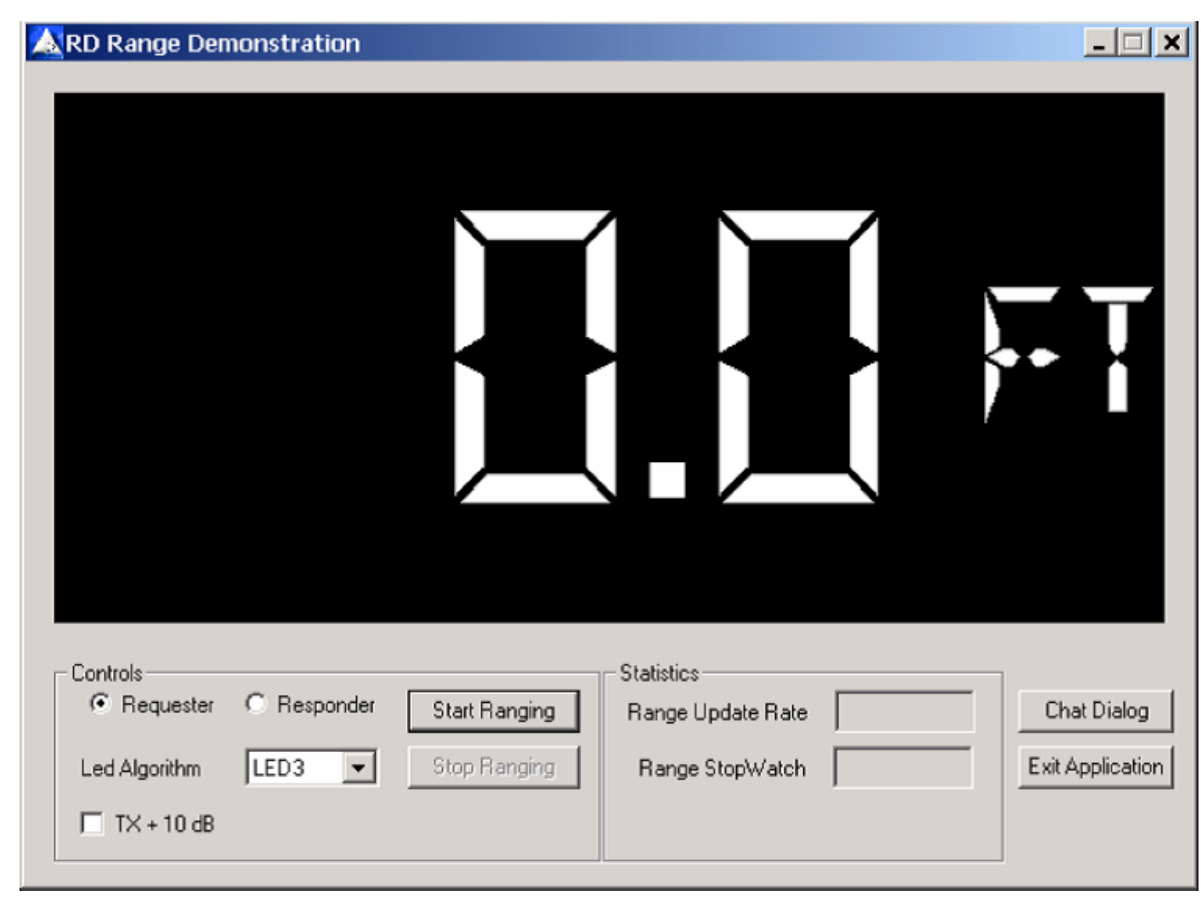

Figure 3.6. Range demonstration application

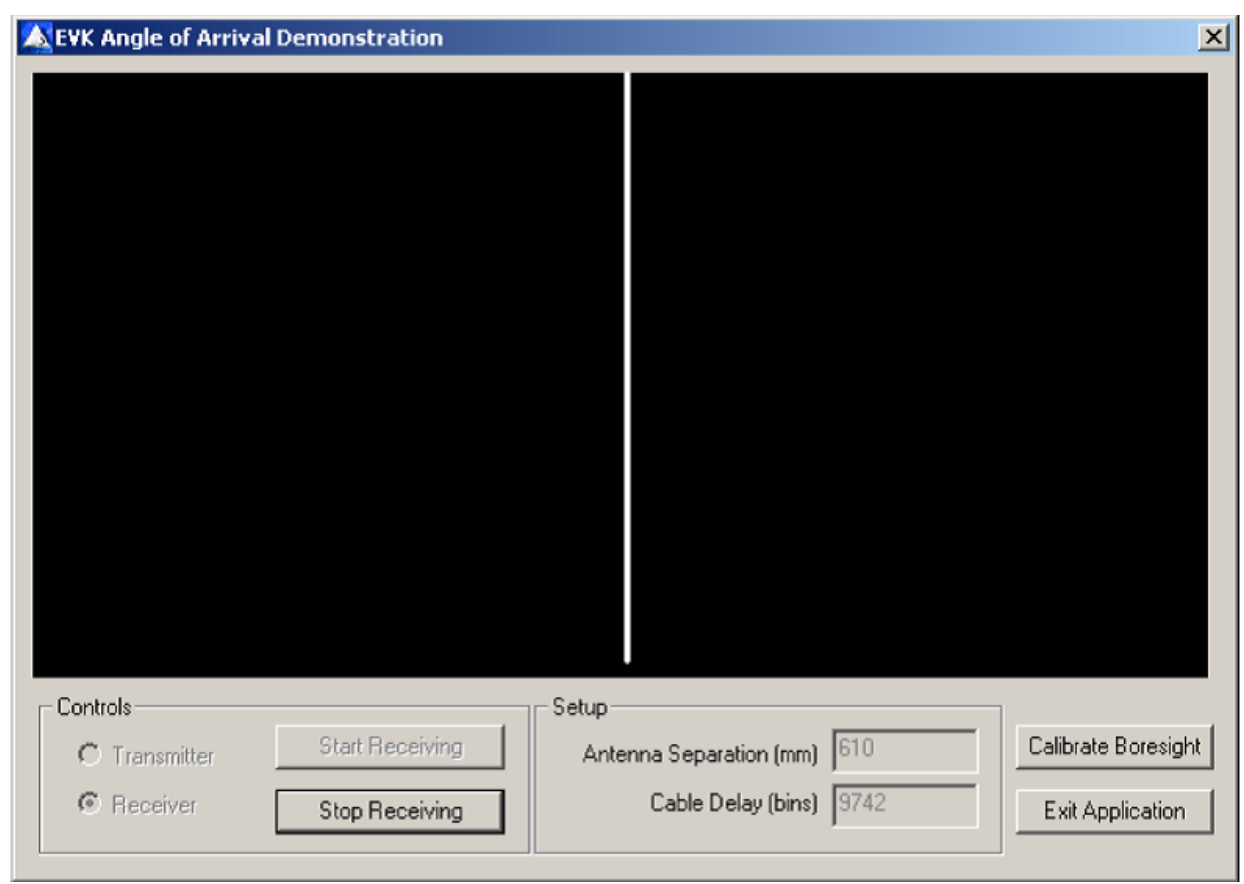

Figure 3.7. Angle of Arrival (AoA) demonstration application 


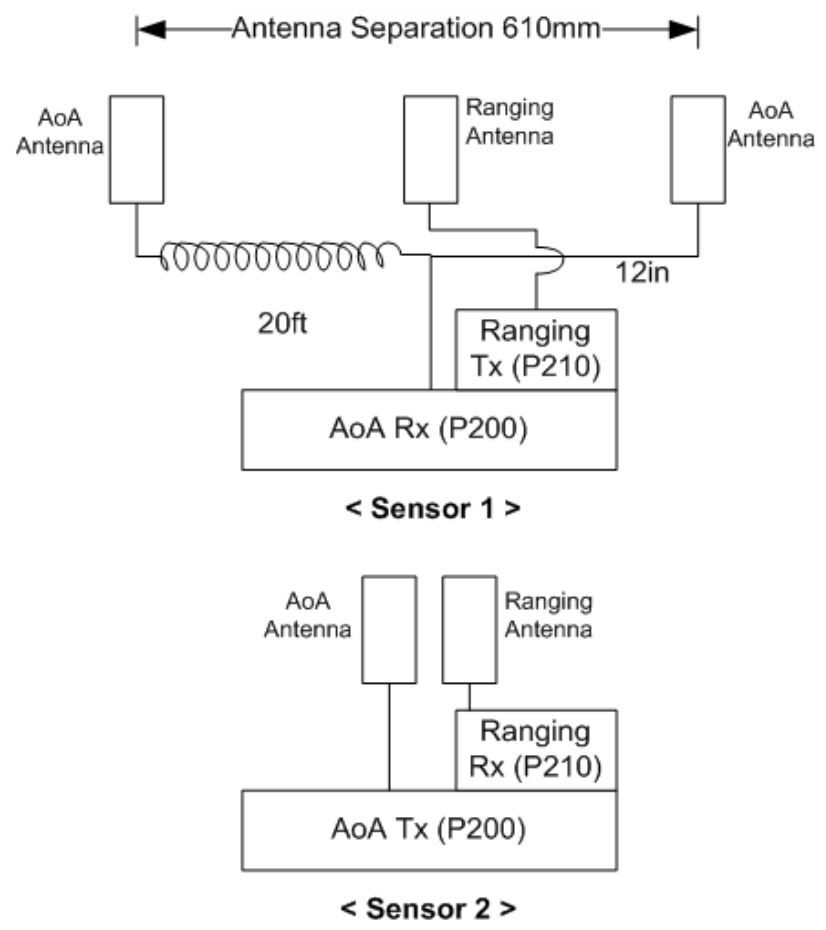

Figure 3.8. Radio setup for sensor 1 and sensor 2 for wall mapping

- Sensor 1 has two antennas for AoA and one antenna for distance ranging. In order to introduce the time difference between reception signals in the AoA receiver antenna array, it has the antenna separation of $61 \mathrm{~cm}$ and the cable lengths of $610 \mathrm{~cm}$ and $30.5 \mathrm{~cm}$ respectively [89]. Figure 3.9 describes the method implemented in the software for AoA calculation. We have converted the antenna separation and the cable lengths into time based on the speed of propagation of radio waves. Consider three cases where the transmitter is located at $0^{\circ}, 90^{\circ}$, and $180^{\circ}$. When the transmitter is at $0^{\circ}$, the signal arrives at antenna B 2 ns ahead of antenna A and therefore the two signals will be received by the radio at times $20-(2+1)=17$ ns apart. If the transmitter is at $90^{\circ}$, the two antennas receive the signal simultaneously but due to different cable lengths the radio receives the signals $20-1=19$ ns 


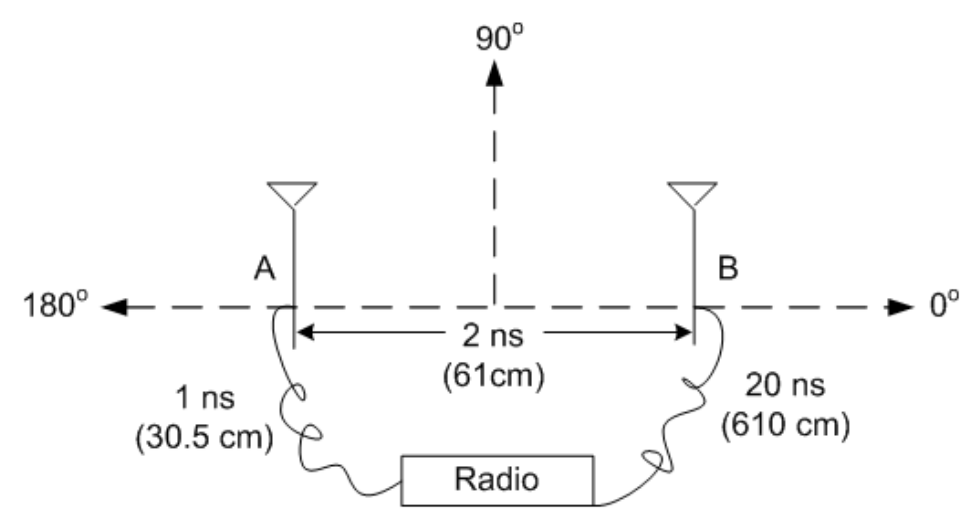

Figure 3.9. Illustration of AoA calculation method

apart. Finally if the transmitter is at $180^{\circ}$, the signals are received by the radio $20+2-1=21 \mathrm{~ns}$ apart. It is easy to see how this idea generalizes to other transmitter angles. The accuracy of determining the transmitter angle depends on the accuracy of the radio in determining the time difference of arrival between the two signals from antennas A and B.

- Sensor 2 has two antennas, one for the AoA measurement and the other for the distance ranging.

The next chapter describes the specifics of the types of measurements conducted for wall identification while chapter 7 describes measurement for wall mapping. 
Chapter 4

\section{EXPERIMENTAL DESIGN FOR WALL IDENTIFICATION}

The fixed and variable experimental parameters for the wall identification research are described below. The fixed parameters do not change for the whole duration of the experiments whereas the variable parameters change to create a set of different test cases.

- Fixed Parameters

- Number of radios: 2

- Transmit power: $-13 \mathrm{dBm}$

- Number of antennas per radio: 1

- Variable Parameters

- Wall types:

* Free space (as a baseline pathloss characterization)

* Dry concrete $4.5^{\prime \prime}$

* Dry concrete $6.5^{\prime \prime}$

* Dry concrete $8^{\prime \prime}$

* Dry reinforced concrete $7.5^{\prime \prime}$

* Dry drywall $0.5^{\prime \prime}$

* Dry plywood $0.5^{\prime \prime}$ 
* Wet concrete $6.5^{\prime \prime}$

* Wet reinforced concrete $7.5^{\prime \prime}$

- Distances between the radios separating the wall:

* 30 different distances between 1 and 3 meters

- Number of repetitions:

* 5 repetitions

- Full factorial experimental design:

* $9 \times 30 \times 5=1350$ total data points

- Metrics

- Received power

\subsection{LABORATORY SETUP}

Figure 4.1 shows the experimental setup used in this research in order to characterize the pathloss of various type and combination of building materials. The transmitter is shown sitting on a stool in the figure while the receiver is in the enclosed box on the right that has shielding material layered on the inside (90 $\mathrm{dB}$ attenuation). The enclosed box door is closed and the door is also shielded with shielding material. Both the transmitter and the receiver radios are placed 1 meter high from the ground. The building material is mounted between the two radios, facing the door of the enclosed box. The building material and the enclosed box were not moved, but the transmitter was put at different distances from the receiver, forming 90 degrees with the building material. The received power at the transmitter was recorded (ten repetitions at each distance) for each placement. 


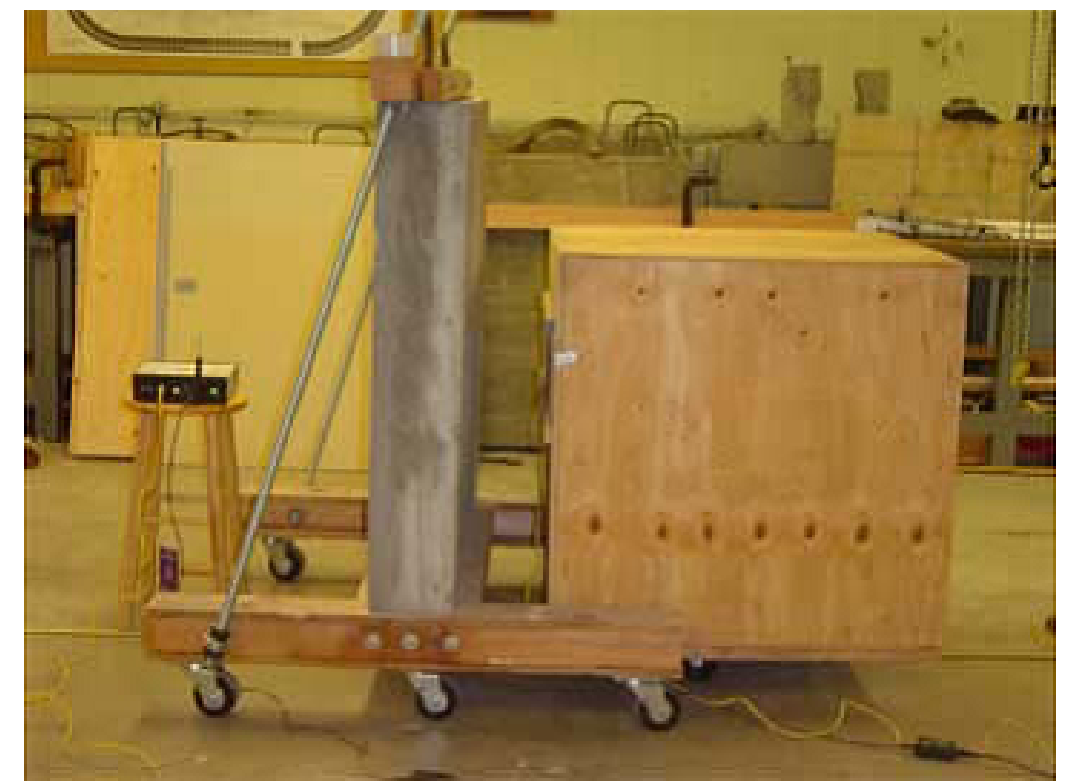

Figure 4.1. Laboratory setup for wall type and thickness measurement

As mentioned earlier, building material types come in a limited set of standard types. Standard types of building materials used in this research include:

- Concrete: $4.5,6.5,8$ inches thick

- Reinforced concrete: 7.5 inches

- Drywall: 0.5 inches

- Plywood: 0.5 inches

As such, building are built with a certain combination of materials. We selected below combinations of material for our research to be as much representative of real world scenarios as possible:

- Freespace (i.e., no building material between the transmitter and the receiver) 
- Concrete \& drywall

- Concrete \& plywood

- Concrete \& plywood \& drywall

- Reinforced concrete \& drywall

- Reinforced concrete \& plywood

- Reinforced concrete \& plywood \& drywall

- A metallic grid \& concrete

Finally, we performed pathloss measurements when building material was dry as well as wet.

These combinations were selected as being representative of real world use cases in the field in terms of combination of material as well as wet conditions. Furthermore, we deliberately used the placement of the radios and material as in Figure 4.1 since in a cavity, Figure 6.1, the two radios will have freespace between themselves and the wall and there will be all kinds of multipath present.

\subsection{MEASUREMENT DATABASE FOR WALL IDENTIFICATION}

We built a database from the measurement data, generated from the above mentioned design and setup. The database records attenuation of UWB signals through different types and thicknesses of walls. This data was used to apply fingerprint algorithms and identify the type of the wall as well as wall thickness as 
explained in chapter 6 . The data from the above mentioned experimental measurement is available at RescueNet website [55] for general use. The Time Domain P200 radios [91] were used for wall identification measurements.

The raw measurement data is searchable using simple queries in the website as shown in Figure 4.2. Sample database query result for raw measurement data are shown in Figure 4.3, with an example of 4.5 inch dry concrete for varying distances between two radios. It can be seen that the distance between the transmitter radio and the receiver radio was varied between 40 inches to 89 inches. The website users can also view analysis results from the raw measurement data such as scatter plot for pathloss analysis of different configurations, e.g., Figure 4.4. 


\section{RESCUENET}

Embedded In-Building Sensor Network to Assist Disaster Rescue

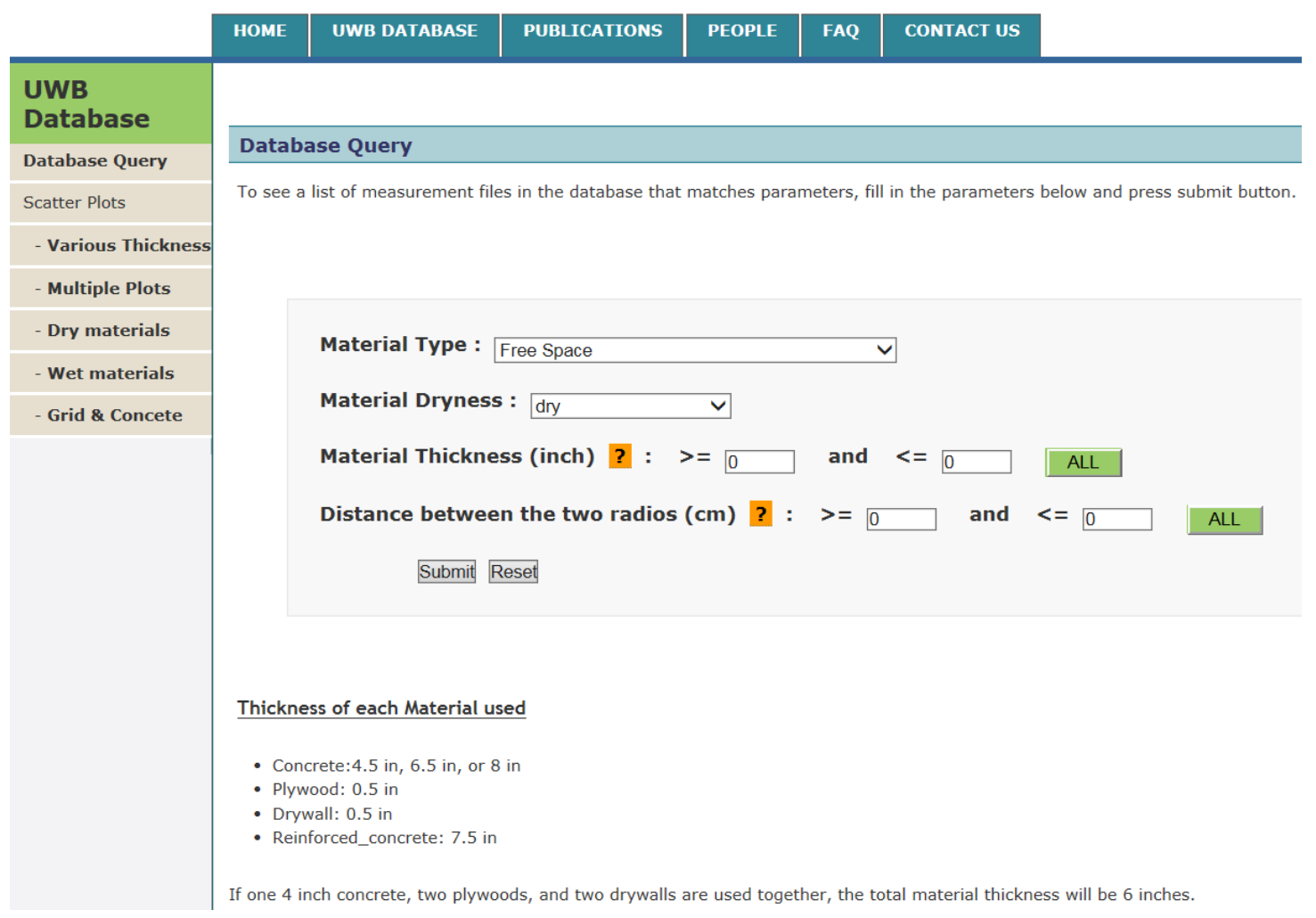

Figure 4.2. RescueNet website for measurement database access 


\section{Query Result}

Number of elements fetched: 30

Each file contains 5 test results, and each column represents one test.

\begin{tabular}{|c|c|c|c|c|c|c|}
\hline Material Type & Dryness & Thickness (inch) & Radio Distance (inch) & Radio Distance $(\mathrm{cm})$ & Data File & Header File \\
\hline Concrete & dry & 4.5 & 40 & 101.6 & | concrete_40_11222005_1.xls & concrete_40_11222005_1.hdr \\
\hline Concrete & dry & 4.5 & 42 & 106.68 & concrete_42_11222005_1.xls & concrete_42_11222005_1.hdr \\
\hline Concrete & dry & 4.5 & 44 & 111.76 & concrete_44_11222005_1.xls & concrete_44_11222005_1.hdr \\
\hline Concrete & dry & 4.5 & 47 & 119.38 & | concrete_47_11222005_1.xls & concrete_47_11222005_1.hdr \\
\hline Concrete & dry & 4.5 & 50 & 127 & |concrete_50_11222005_1.xls & concrete_50_11222005_1.hdr \\
\hline Concrete & dry & 4.5 & 53 & 134.62 & concrete_53_11222005_1.xls & concrete_53_11222005_1.hdr \\
\hline Concrete & dry & 4.5 & 55 & 139.7 & concrete_55_11222005_1.xls & concrete_55_11222005_1.hdr \\
\hline Concrete & dry & 4.5 & 58 & 147.32 & |concrete_58_11222005_1.xls & concrete_58_11222005_1.hdr \\
\hline Concrete & dry & 4.5 & 60 & 152.4 & concrete_60_11222005_1.xls & concrete_60_11222005_1.hdr \\
\hline Concrete & dry & 4.5 & 62 & 157.48 & concrete_62_11222005_1.xls & concrete_62_11222005_1.hdr \\
\hline Concrete & dry & 4.5 & 65 & 165.1 & | concrete_65_11222005_1.xis & concrete_65_11222005_1.hdr \\
\hline Concrete & dry & 4.5 & 68 & 172.72 & |concrete_68_11222005_1.xls & concrete_68_11222005_1.hdr \\
\hline Concrete & dry & 4.5 & 71 & 180.34 & ||concrete_71_11222005_1.xls & concrete_71_11222005_1.hdr \\
\hline Concrete & dry & 4.5 & 72 & 182.88 & | concrete_72_11222005_1.xls & concrete_72_11222005_1.hdr \\
\hline Concrete & dry & 4.5 & 74 & 187.96 & |concrete_74_11222005_1.xls & concrete_74_11222005_1.hdr \\
\hline Concrete & dry & 4.5 & 76 & 193.04 & | concrete_76_11222005_1.xls & concrete_76_11222005_1.hdr \\
\hline Concrete & dry & 4.5 & 79 & 200.66 & |concrete_79_11222005_1.xls & concrete_79_11222005_1.hdr \\
\hline Concrete & dry & 4.5 & 81 & 205.74 & concrete_81_11222005_1.xls & concrete_81_11222005_1.hdr \\
\hline Concrete & dry & 4.5 & 83 & 210.82 & concrete_83_11222005_1.xls & concrete_83_11222005_1.hdr \\
\hline Concrete & dry & 4.5 & 86 & 218.44 & | concrete_86_11222005_1.xls & concrete_86_11222005_1.hdr \\
\hline Concrete & dry & 4.5 & 89 & 226.06 & concrete_89_11222005_1.xls & concrete_89_11222005_1.hdr \\
\hline
\end{tabular}

Figure 4.3. Sample database query result 


\section{RESCUENET}

\section{Embedded In-Building Sensor Network to Assist Disaster Rescue}

\begin{tabular}{|c|c|c|c|c|c|c|}
\hline & HOME & UWB DATABASE & PUBLICATIONS & PEOPLE & FAQ & CONTACT US \\
\hline $\begin{array}{l}\text { UWB } \\
\text { Database }\end{array}$ & & & & & & \\
\hline Database Query & \multicolumn{6}{|c|}{ Scatter Plots } \\
\hline Scatter Plots & \multicolumn{6}{|c|}{ Figures of various thickness } \\
\hline - Various Thickness & \multicolumn{6}{|c|}{ 1. 4.5" Concrete with other materials (dry) } \\
\hline - Multiple Plots & & & & & & \\
\hline - Dry materials & \multicolumn{6}{|c|}{ 2. 6.5" Concrete with other materials (dry) } \\
\hline - Wet materials & \multirow{2}{*}{\multicolumn{6}{|c|}{ 3. 8" Concrete with other materials (dry) }} \\
\hline - Grid \& Concete & & & & & & \\
\hline & 1. $4.5^{\prime \prime}$ & crete with other $n$ & terials (dry) & & & \\
\hline
\end{tabular}

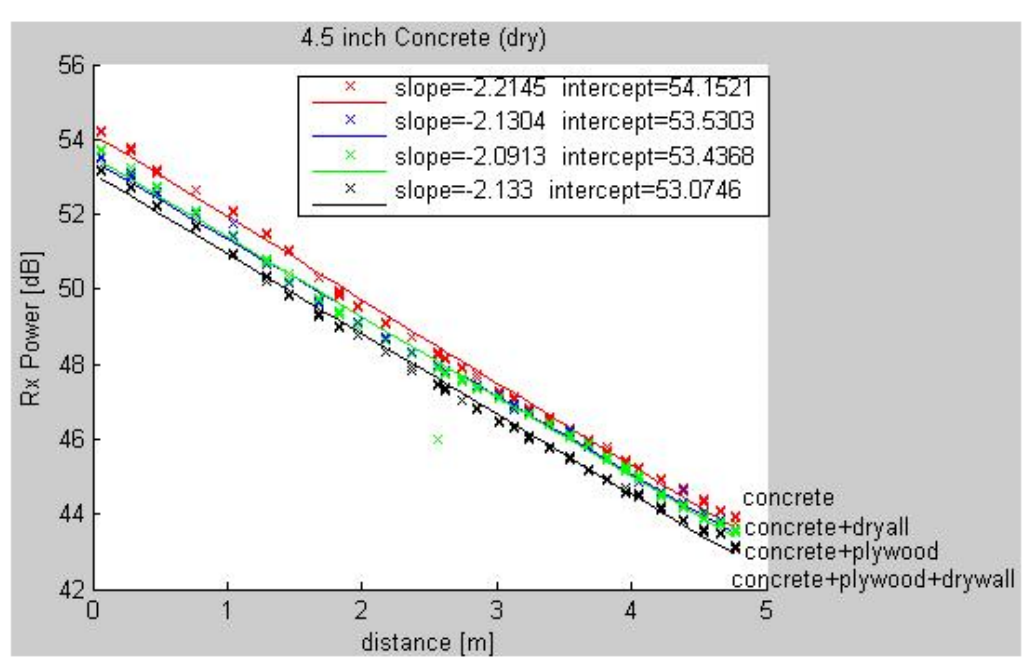

[Back to TOP]

Figure 4.4. Example database analysis on RescueNet website 
Chapter 5

\section{RESULTS FOR WALL TYPE AND THICKNESS MEASUREMENT}

In this chapter we present pathloss measurement results for wall type and thickness identification for various test configurations.

\subsection{FREESPACE PATHLOSS AS BASELINE}

Freespace measurement serves as the baseline for all other measurements with building material for comparison. Its pathloss plot should show highest Rx Power compared to results using some material obstruction. Figure 5.1 shows a scatter plot of measurement results when no building material was placed between the two radios. The best fit line is drawn along those scatter data points to obtain its pathloss line with slope and $y$-intercept. As distance between two radios gets larger, the variation of measurement data increased.

\subsection{PATHLOSS RESULT FOR DRY MATERIAL}

\subsubsection{Pathloss Result for Dry Concrete and Other Material}

This section shows pathloss result for $6.5^{\prime \prime}$ dry concrete as well as with other material. Figure 5.2, Figure 5.3, Figure 5.4 and Figure 5.5 show pathloss graph for dry concrete in combination with following material:

(a) Dry concrete only 


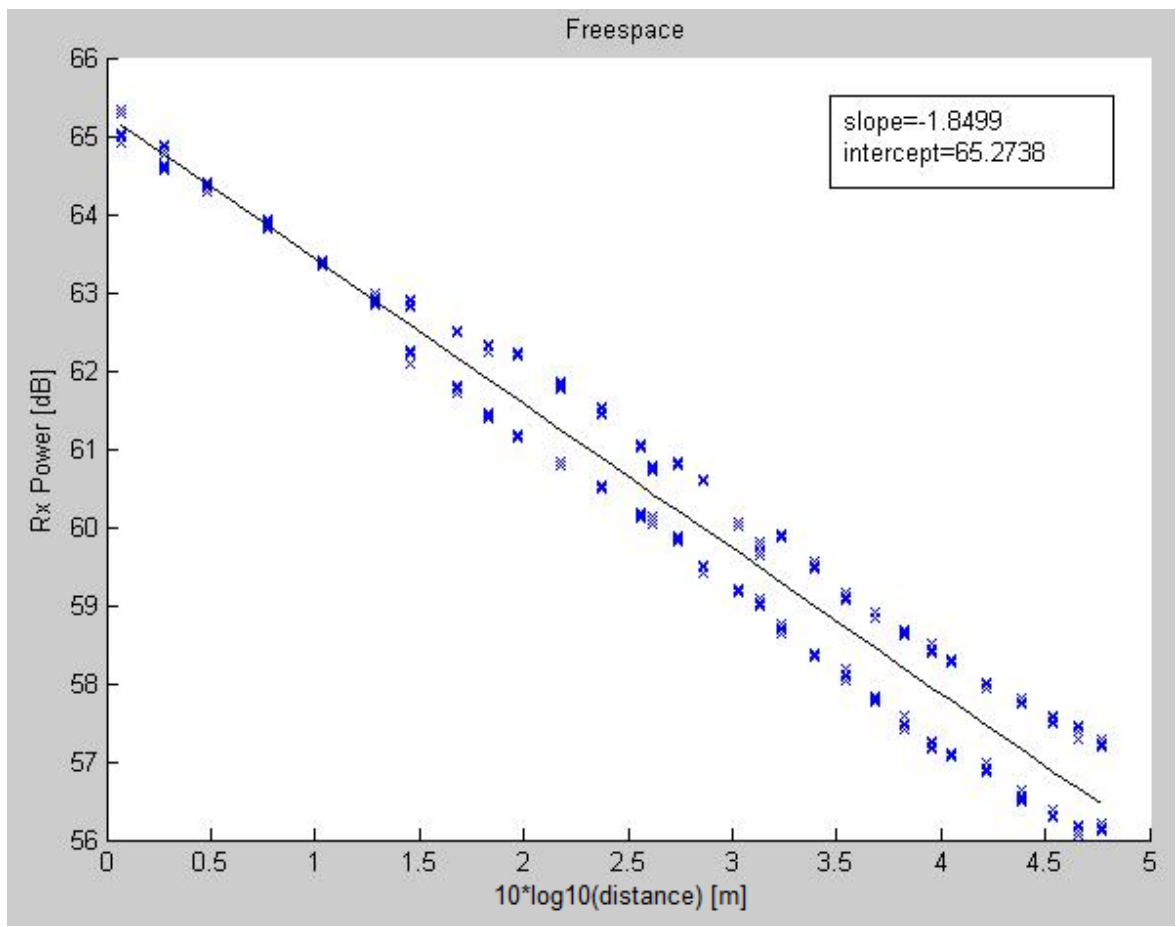

Figure 5.1. Freespace pathloss

(b) Dry concrete with dry plywood

(c) Dry concrete with dry drywall

(d) Dry concrete with dry plywood and dry drywall

In order to see how the individual plots relate to each other, we put them into one plot as in Figure 5.6, where it can be seen that the signal attenuation is largest when all materials were put together (i.e., option (a)) compared to other combinations. When either plywood or drywall were put together with concrete, it does not effectively attenuate the signal compared to (a) concrete only. Thus it can sometimes appear even better than (a). Since measurement was done in different days, it is probable that it was affected by weather changes as well as slight material placement changes during lab test setup. Overall, the pathloss 


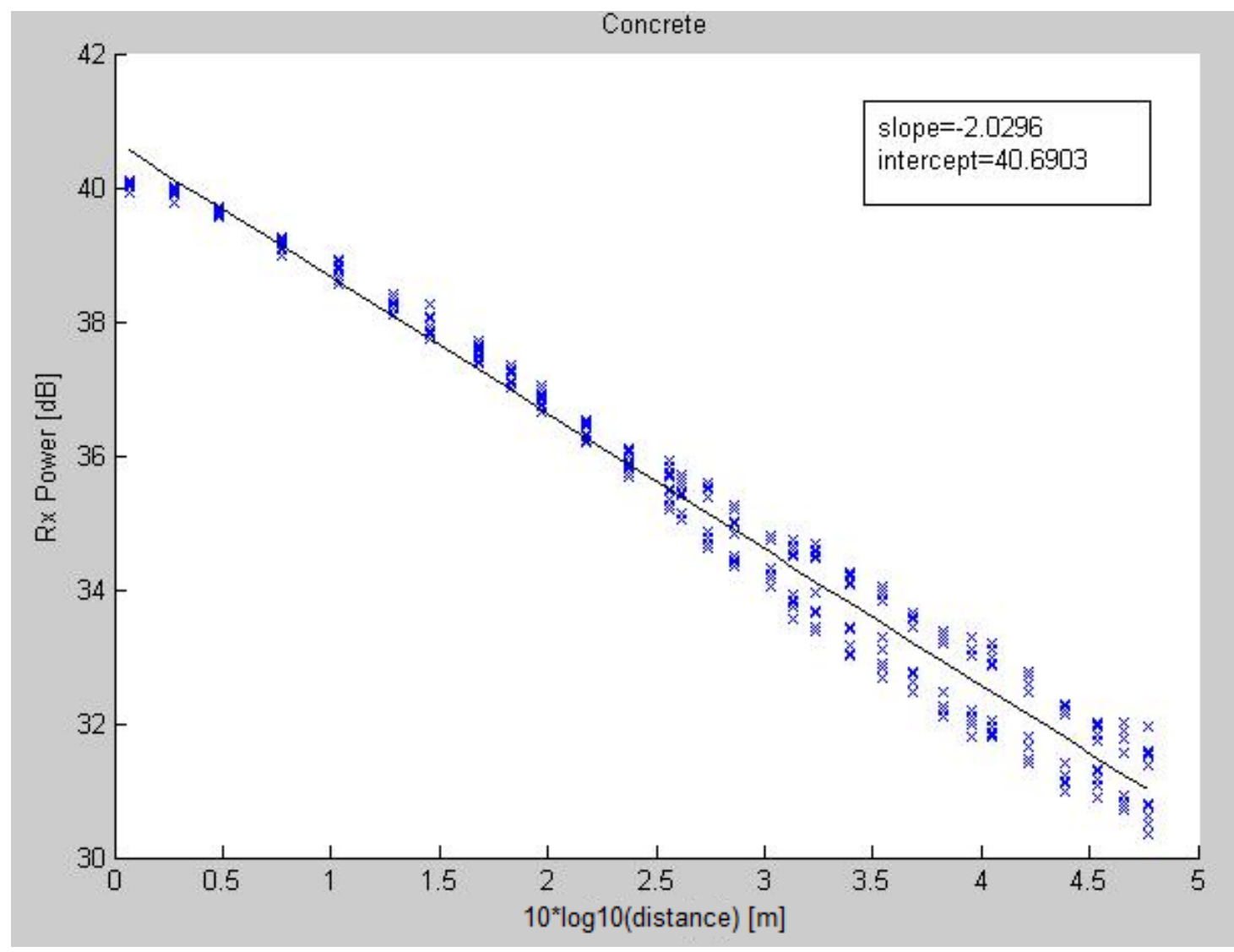

Figure 5.2. $6^{\prime \prime}$ dry concrete pathloss

graph of (b) and (c) still stays close to that of (a) dry concrete, and it is well separated from either freespace or reinforced concrete pathloss graphs.

\subsubsection{Pathloss Result for Dry Reinforced Concrete and Other Material}

This section shows pathloss result for $7.5^{\prime \prime}$ dry reinforced concrete as well as with other material. Figure 5.7, Figure 5.8, Figure 5.9 and Figure 5.10 show pathloss graph for dry reinforced concrete in combination with following material:

(a) Dry reinforced concrete only

(b) Dry reinforced concrete with dry plywood 


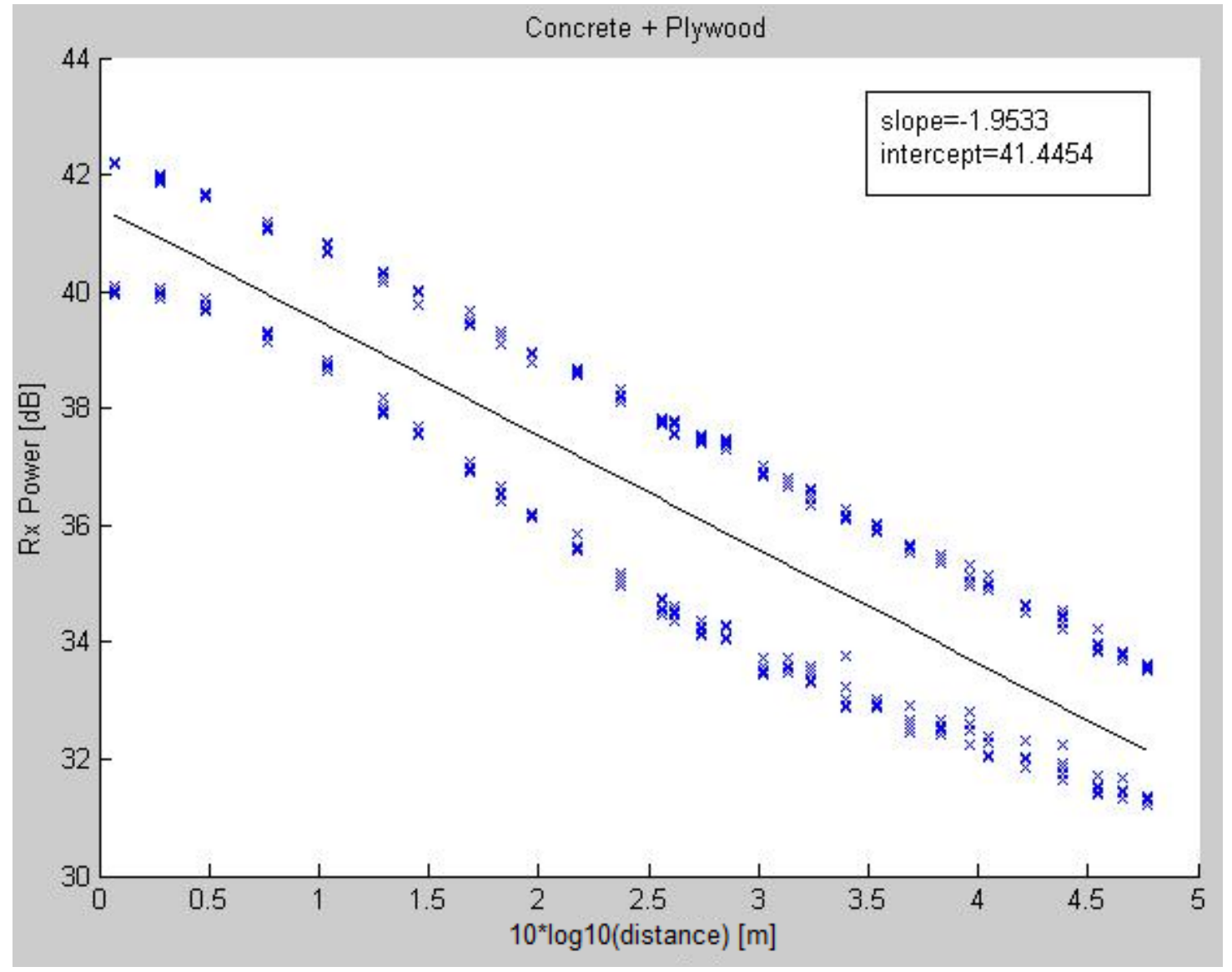

Figure 5.3. $6^{\prime \prime}$ dry concrete with plywood 


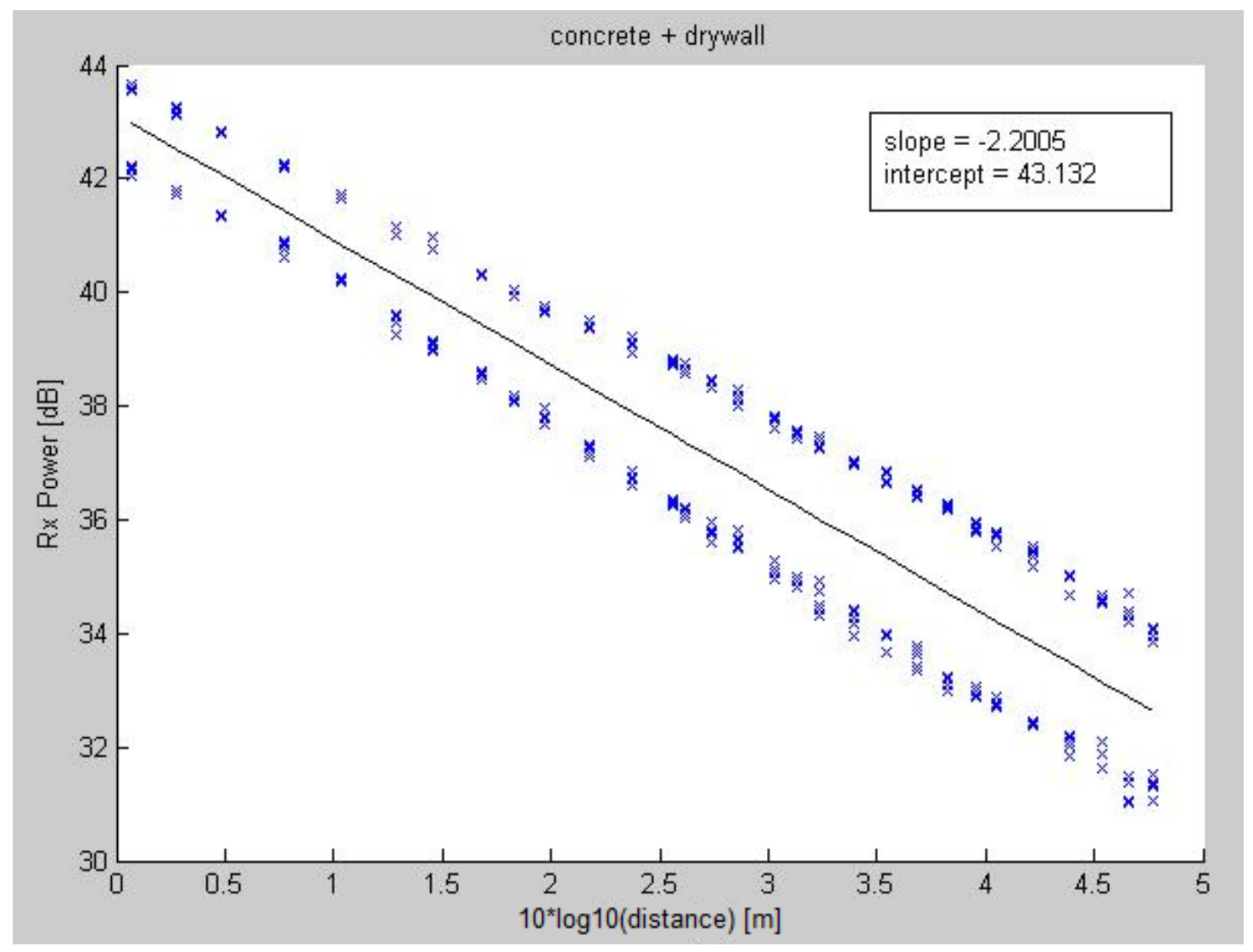

Figure 5.4. Dry concrete with drywall 


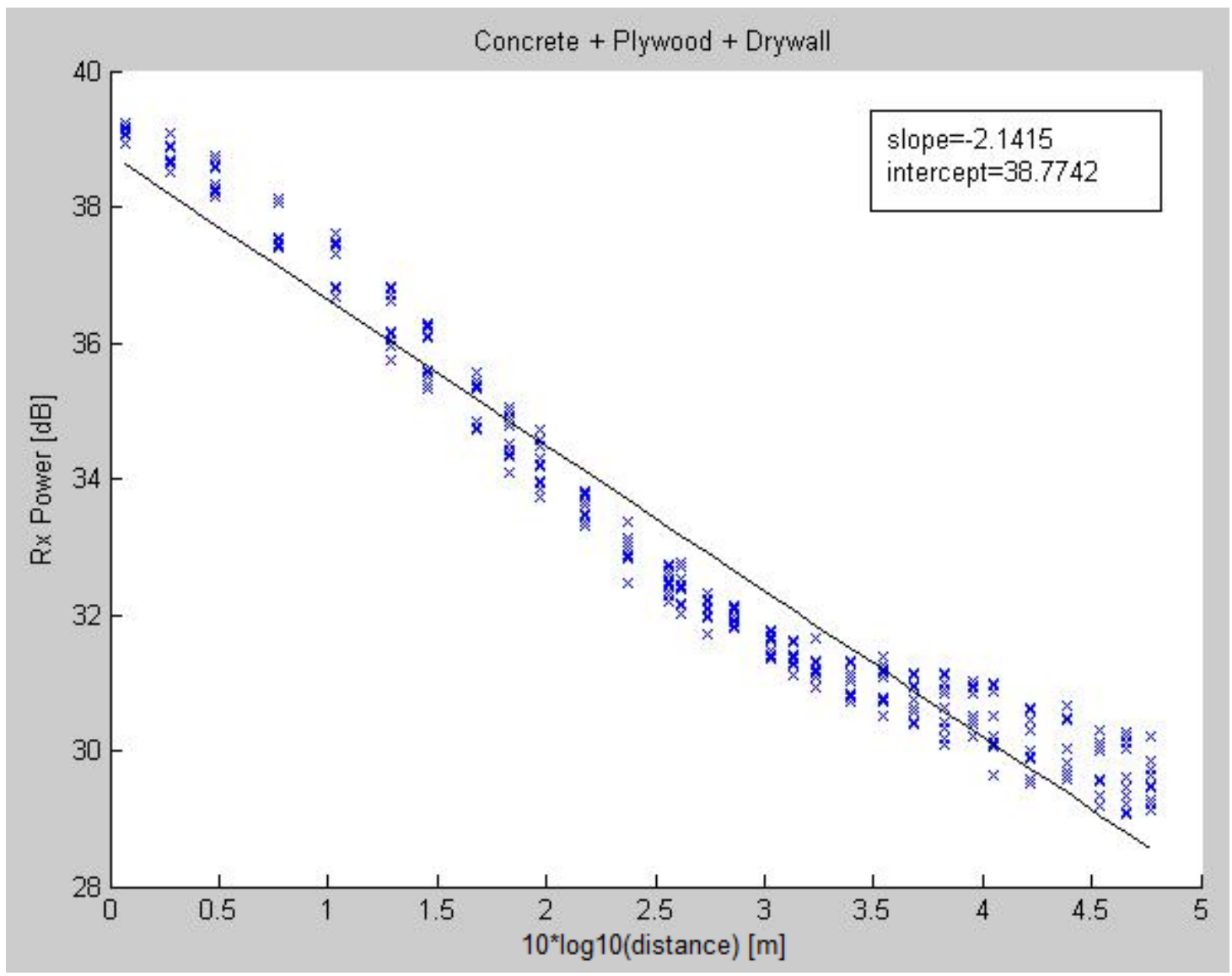

Figure 5.5. Dry concrete with plywood and drywall 


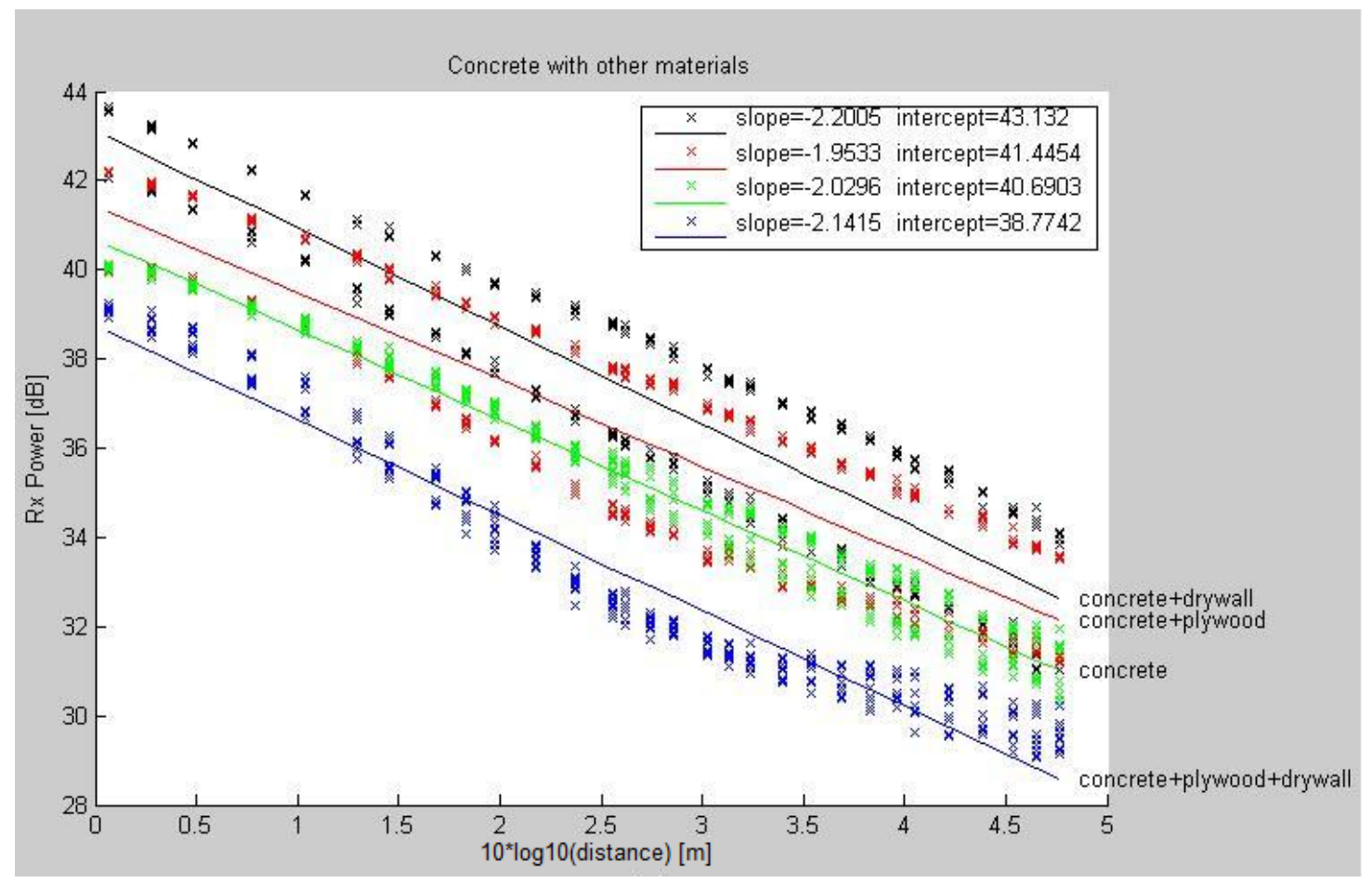

Figure 5.6. Dry concrete with other material

(c) Dry reinforced concrete with dry drywall

(d) Dry reinforced concrete with dry plywood and dry drywall

In order to see how the above individual plots are relative to each other, we put them into one plot as in Figure 5.11, where it can be seen that the signal attenuation is largest when all materials were put together (i.e., option (a)) compared to other combinations. When either plywood or drywall were put together with concrete, it does not effectively attenuate the signal compared to (a) reinforced concrete only. Thus, it can sometimes appear even better than (a). As explained in section 5.2.1, since measurement was done in different days, it is probable that it was affected by weather changes as well as slight material placement changes during lab test setup. Overall, the pathloss graph of (b) and (c) still stays close to that of (a) 


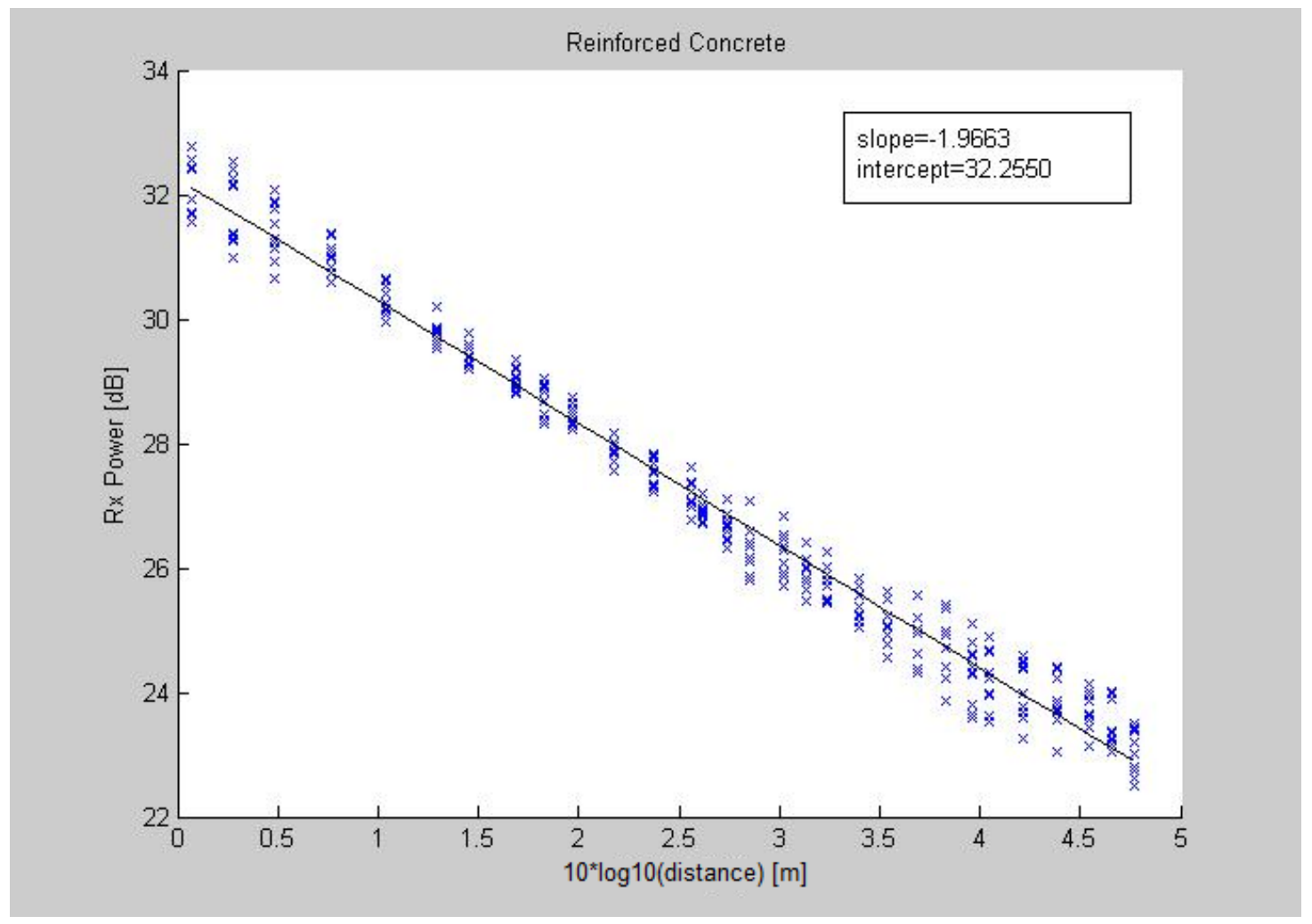

Figure 5.7. Dry reinforced concrete pathloss

reinforced concrete only. It is well separated from either freespace or reinforced concrete pathloss graphs.

\subsubsection{Dry Concrete of Other Thickness}

This section shows pathloss plots when concrete of various thickness was placed in-between two radios as well as when it was placed back to back with other materials. The concrete thickness for Figure 5.12 and Figure 5.13 is 4.5 inches and 8 inches respectively. The combination of material used for measurement was the same as previous experiments.

The following observations can be made from the pathloss plots: 


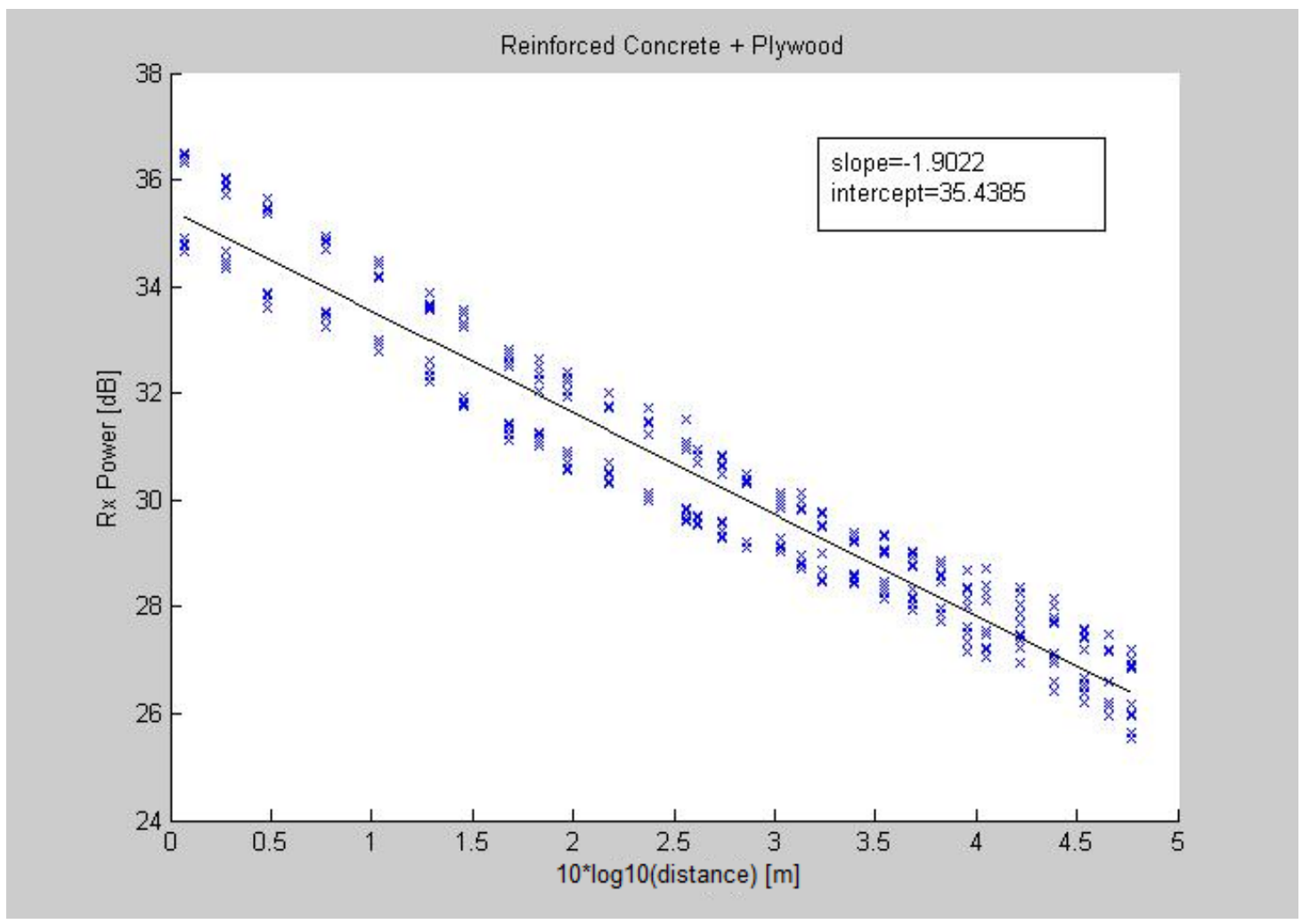

Figure 5.8. Dry reinforced concrete with plywood 


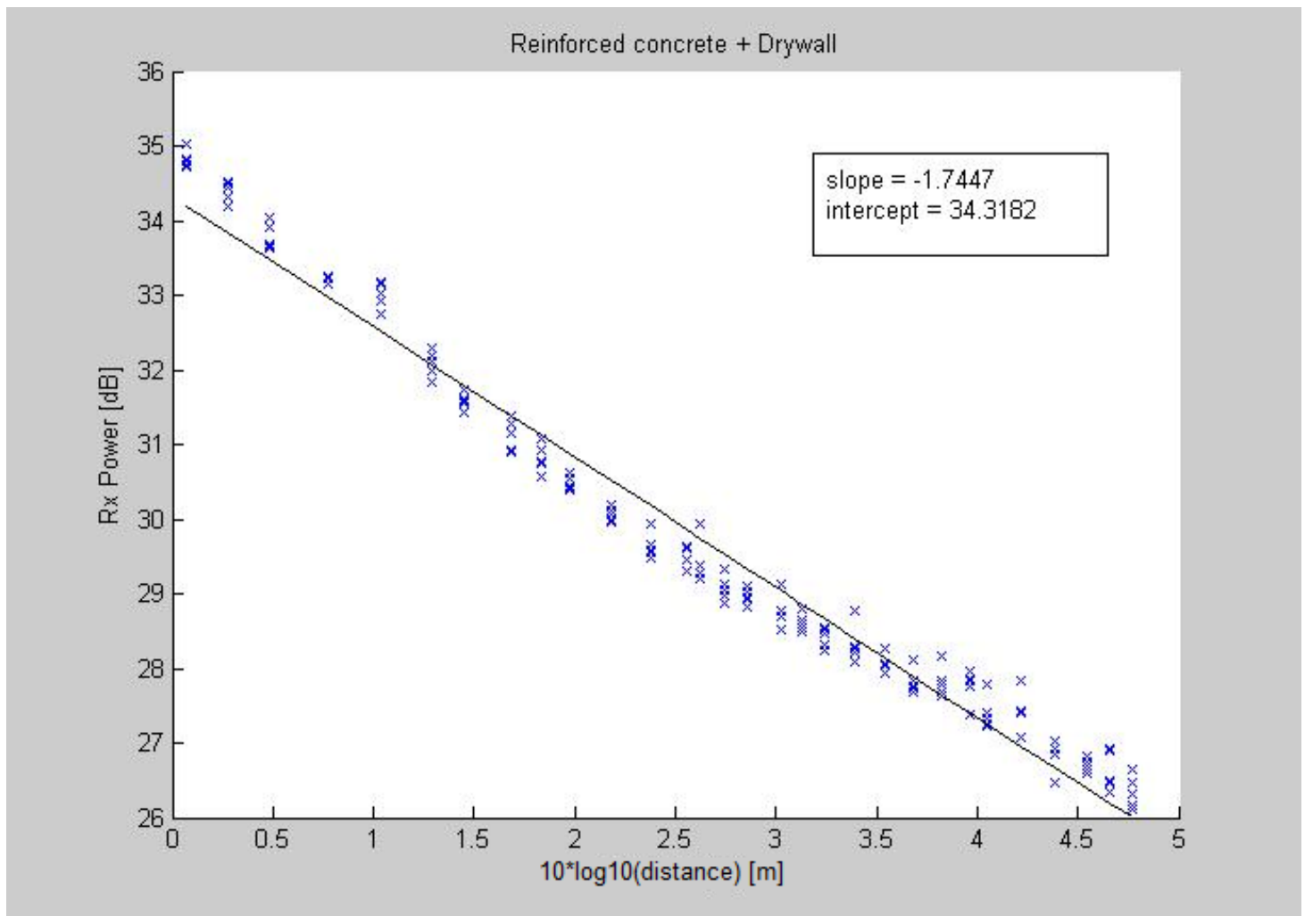

Figure 5.9. Dry reinforced concrete with drywall 


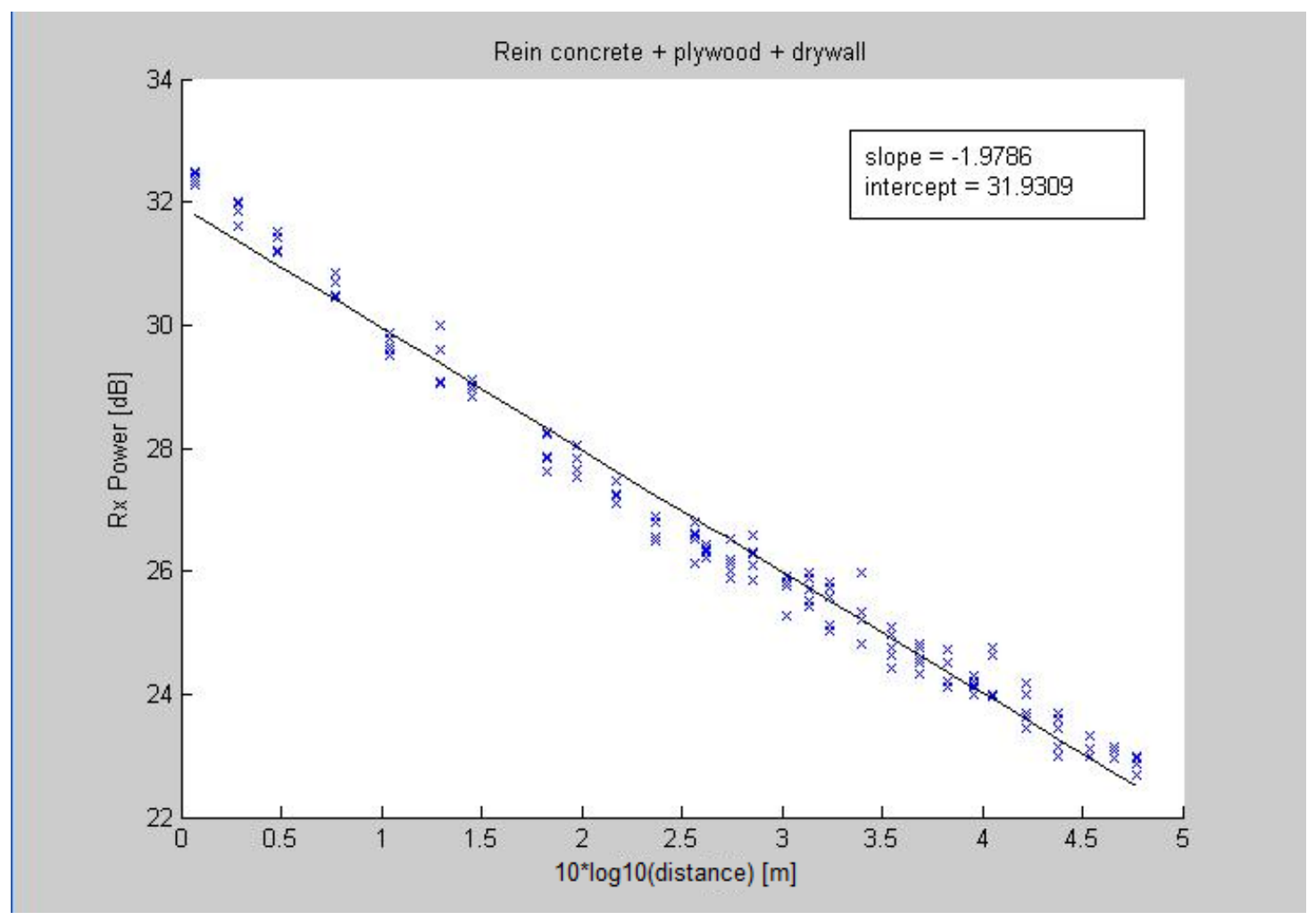

Figure 5.10. Dry reinforced concrete with plywood and drywall 


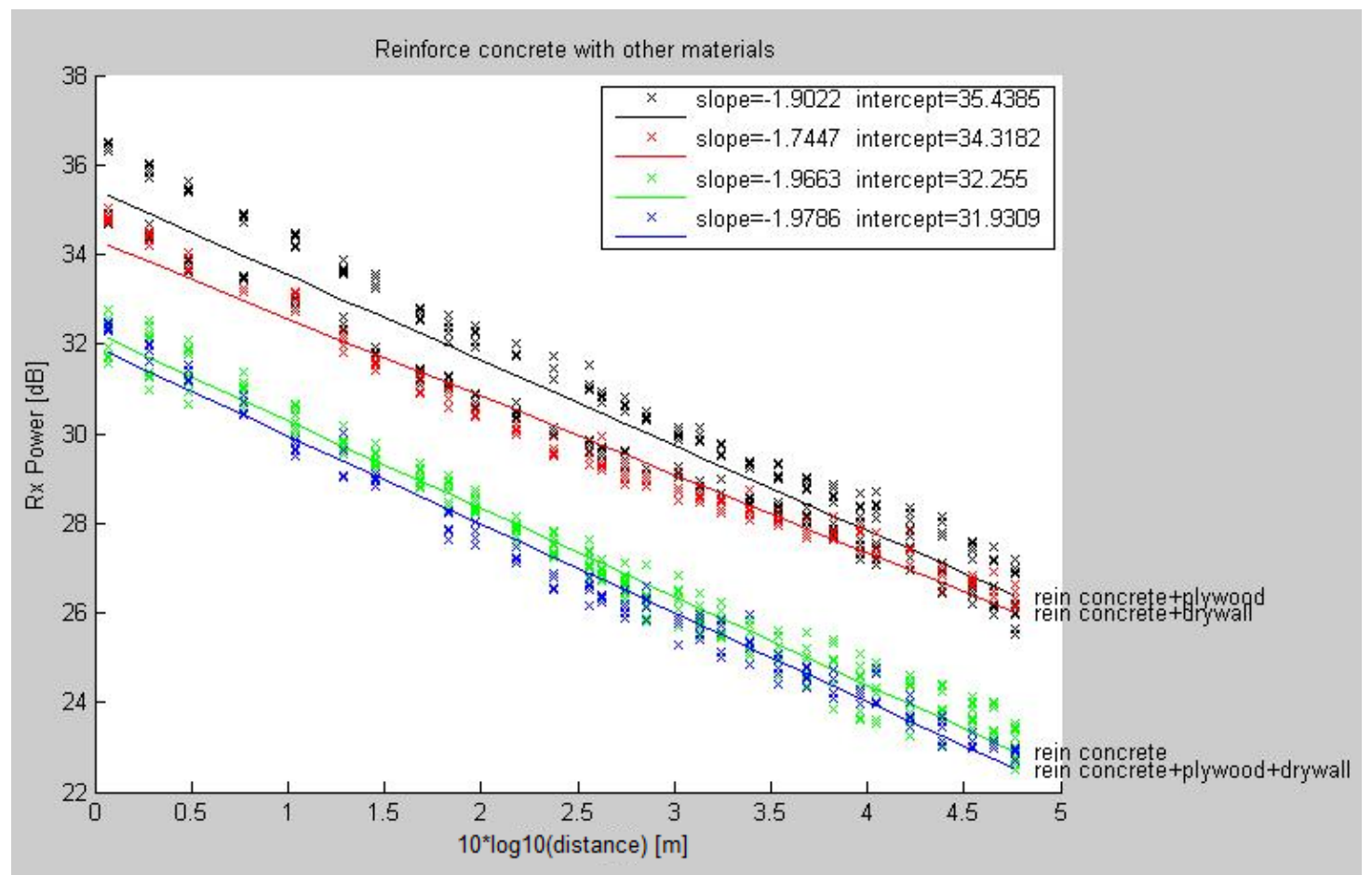

Figure 5.11. Dry reinforced concrete with other material

- The legend in the figures show the slope and $y$-intercept of each pathloss plot. As shown in the below figures, as concrete gets thicker from 4.5 inches to 8 inches the received power gets weaker, and thus $y$-intercept becomes smaller and slope got steeper.

- In general, when more materials were used together with concrete the signal attenuation got higher, thus the $y$-intercept of concrete with other material was lower than concrete only. 


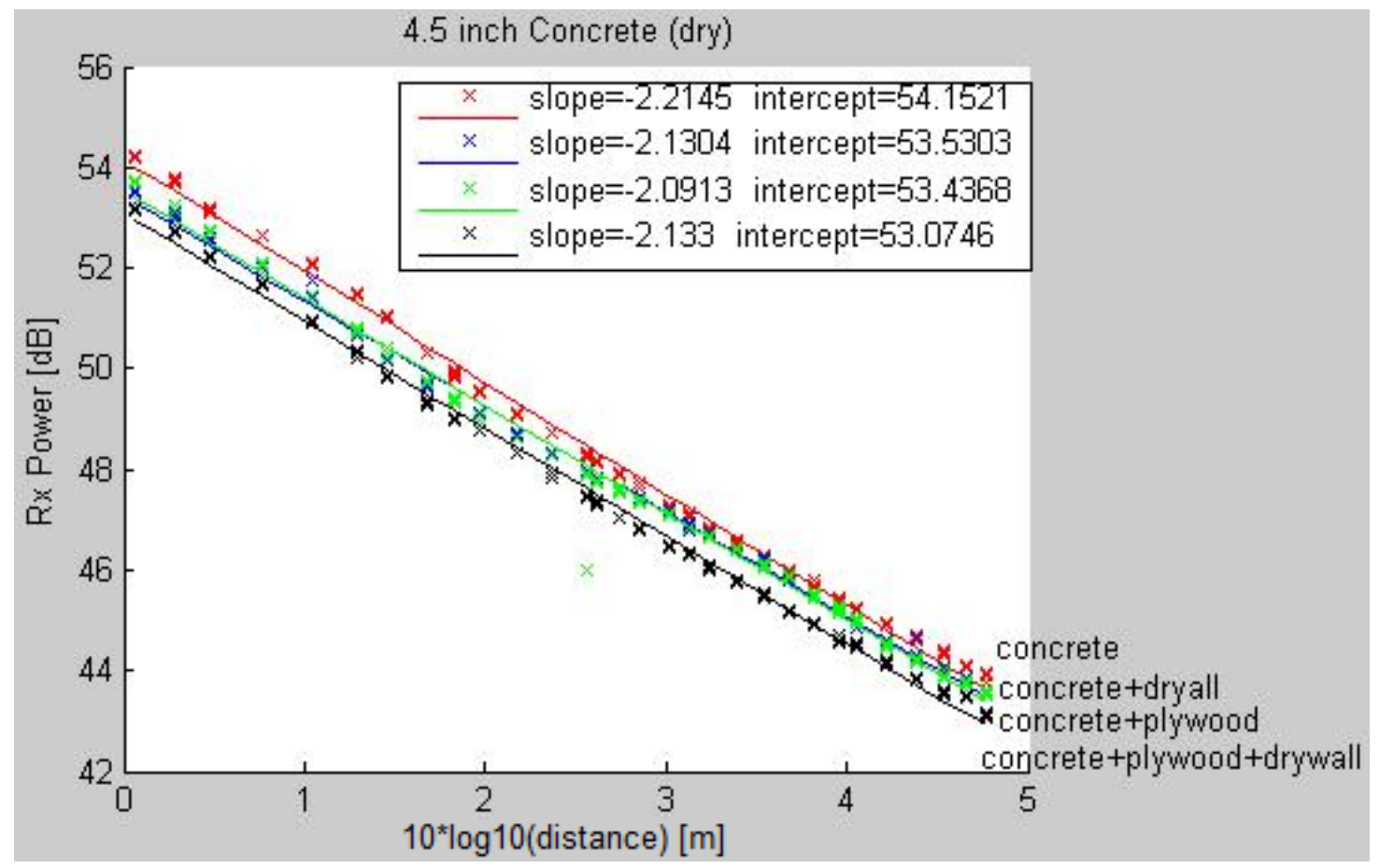

Figure 5.12. 4.5" dry concrete with other materials

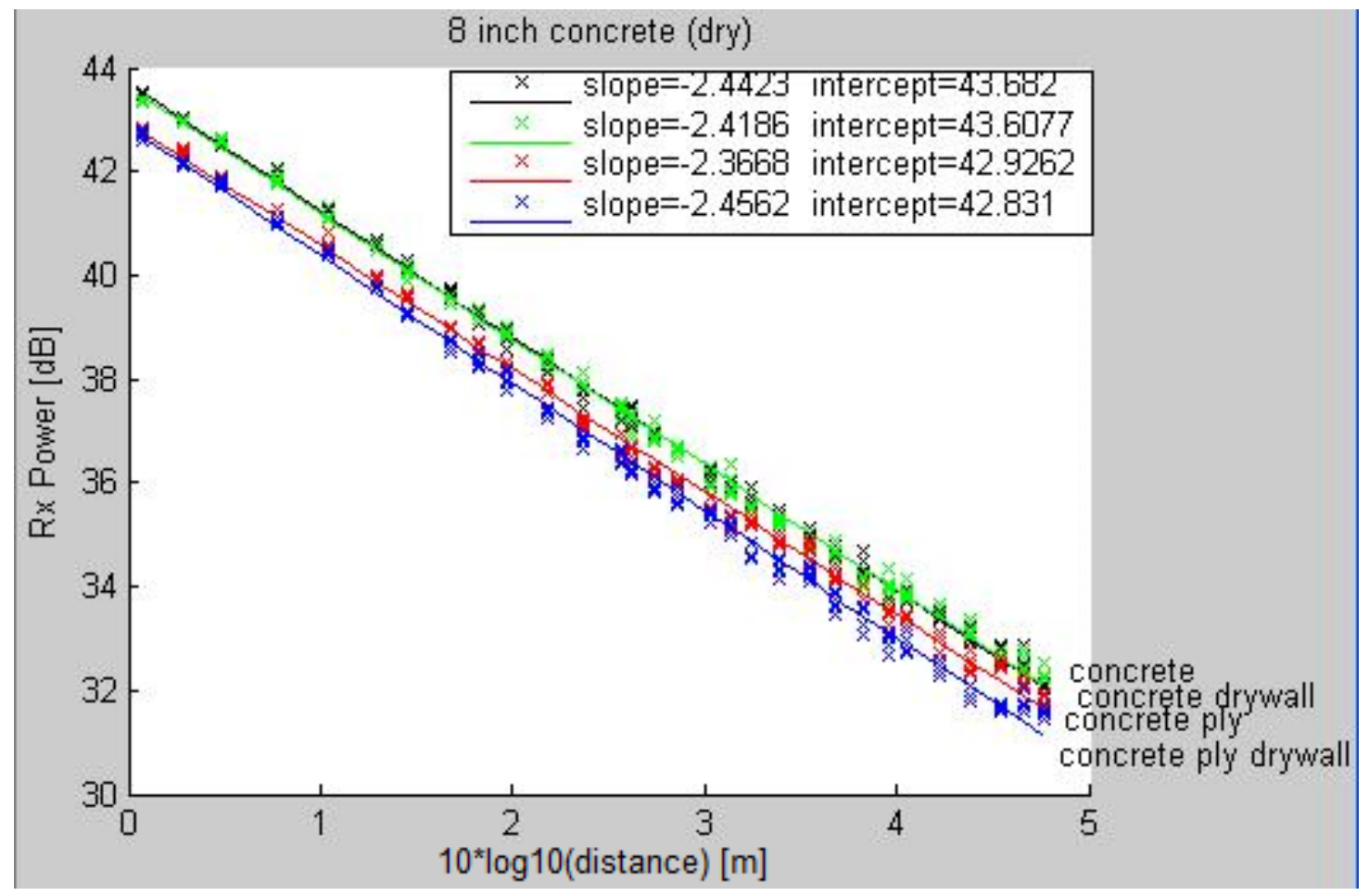

Figure 5.13. $8^{\prime \prime}$ dry concrete with other materials 


\subsection{PATHLOSS RESULT FOR WET MATERIAL}

\subsubsection{Pathloss Result for Wet Concrete and Other Material}

This section shows pathloss result for $6.5^{\prime \prime}$ wet concrete as well as with other material. Figure 5.14, Figure 5.15, Figure 5.16 and Figure 5.17 show pathloss graph for dry concrete in combination with following material:

(a) Wet concrete only

(b) Wet concrete with wet plywood

(c) Wet concrete with dry drywall

(d) Wet concrete with wet plywood and dry drywall

Above plots were put into one plot in Figure 5.18. Wet concrete result is similar to dry concrete result, where the signal attenuation is largest when all materials were put together (i.e., option (d)).

\subsubsection{Pathloss Result for Wet Reinforced Concrete and Other Material}

This section shows pathloss result for wet reinforced concrete as well as with other material. Figure 5.19, Figure 5.20, Figure 5.21, and Figure 5.22 show pathloss graph for wet reinforced concrete in combination with following material:

(a) Wet reinforced concrete only

(b) Wet reinforced concrete with wet plywood

(c) Wet reinforced concrete with dry drywall

(d) Wet reinforced concrete with wet plywood and dry drywall 


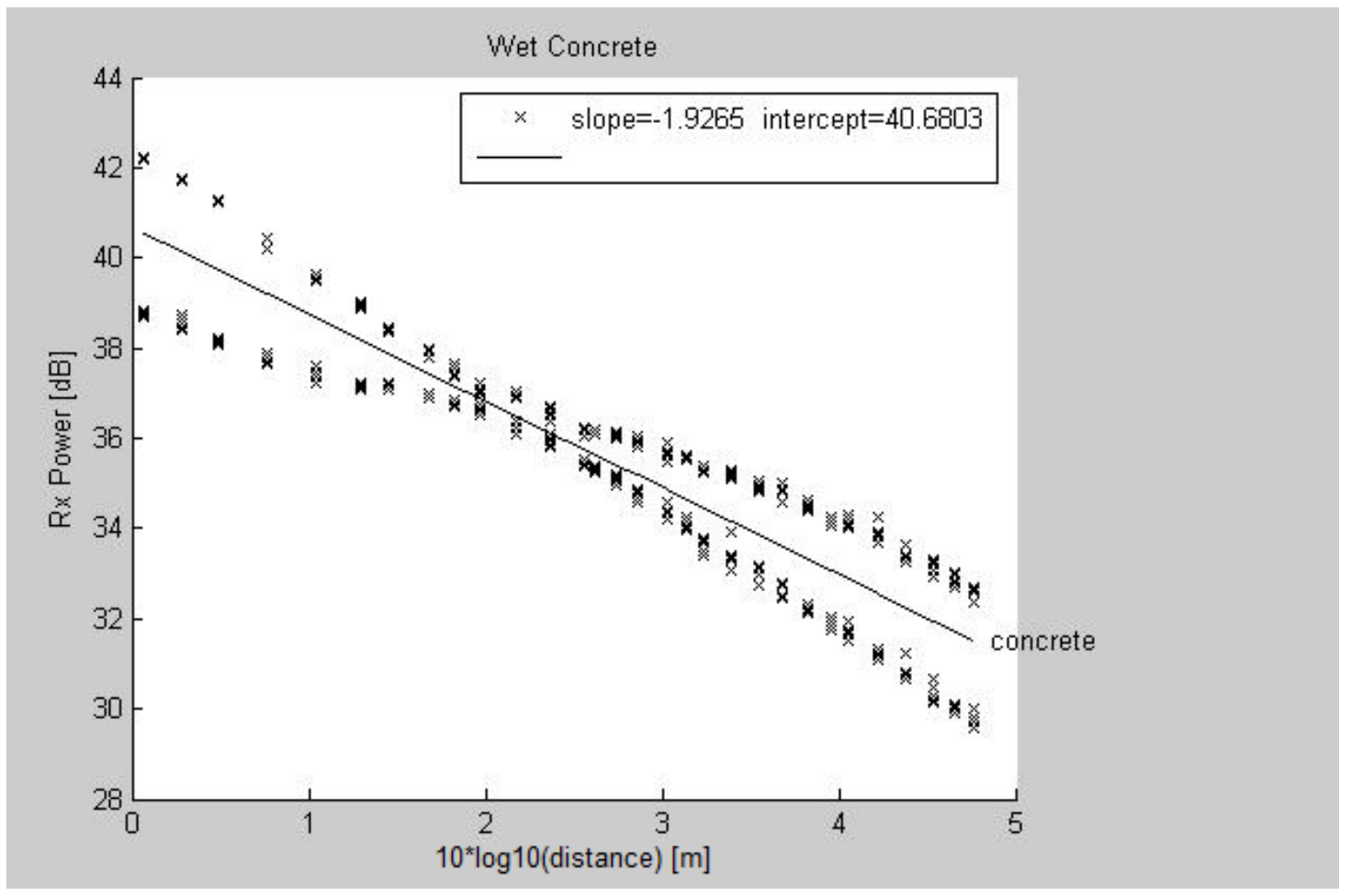

Figure 5.14. Wet concrete pathloss 


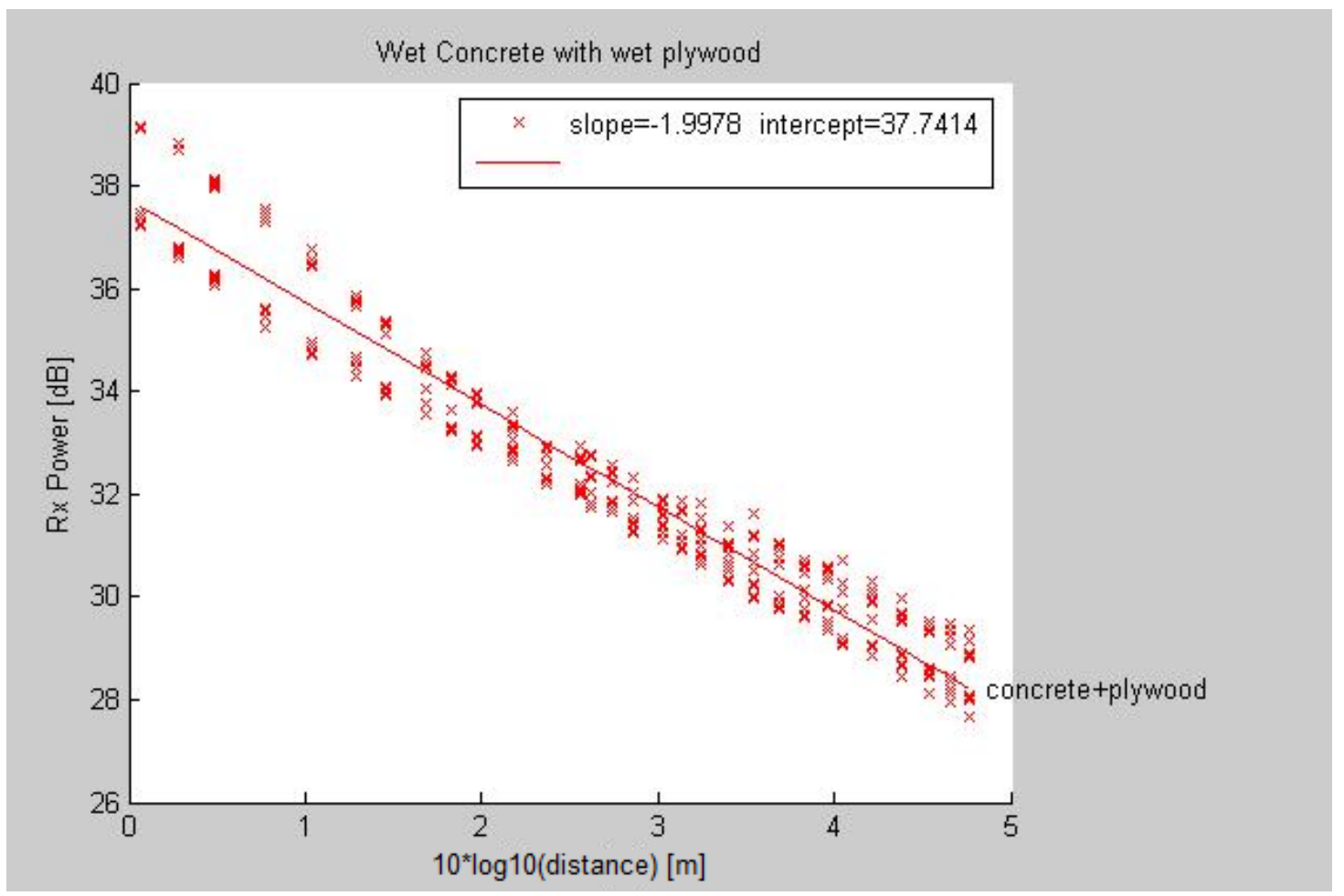

Figure 5.15. Wet concrete with wet plywood 


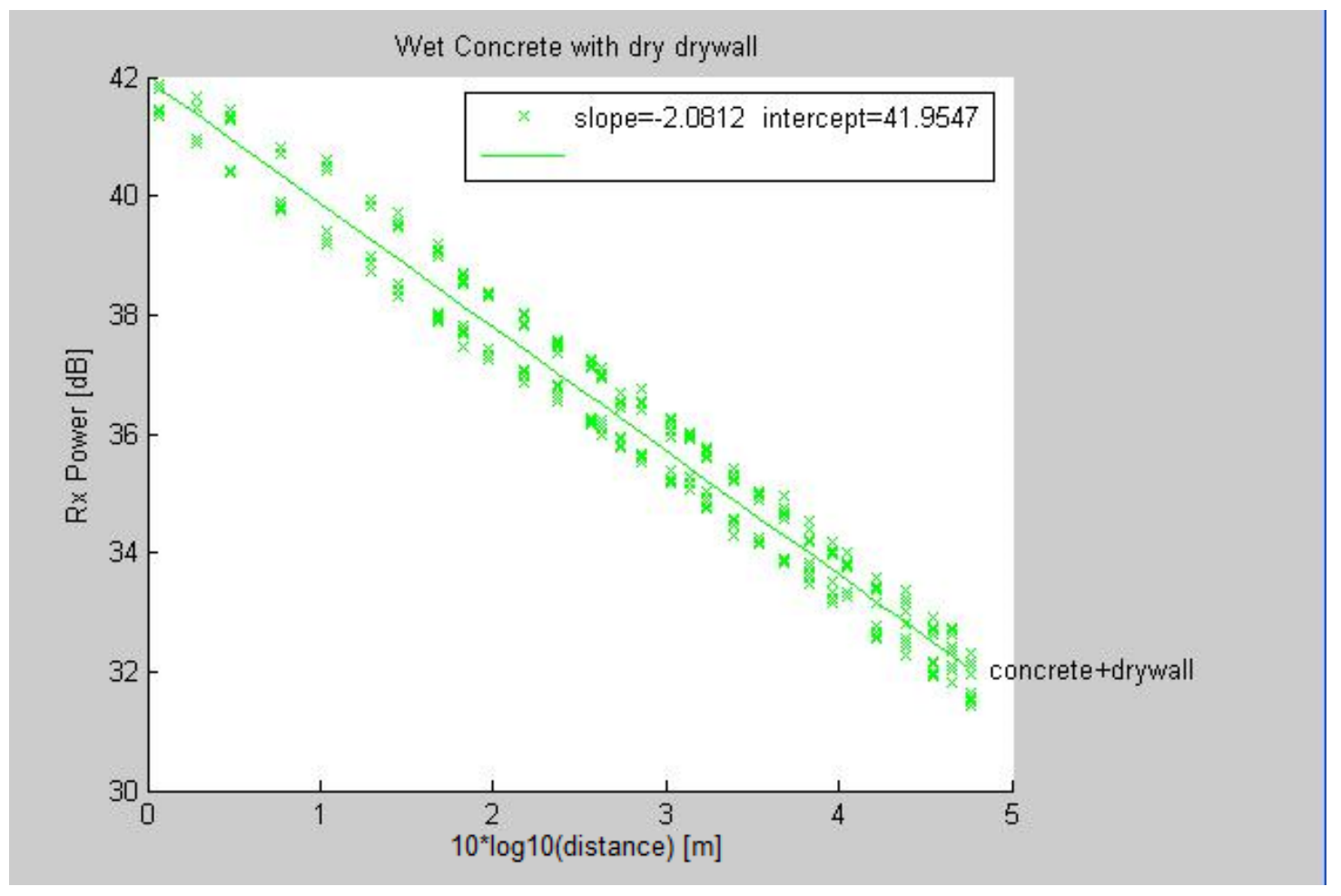

Figure 5.16. Wet concrete with dry drywall 


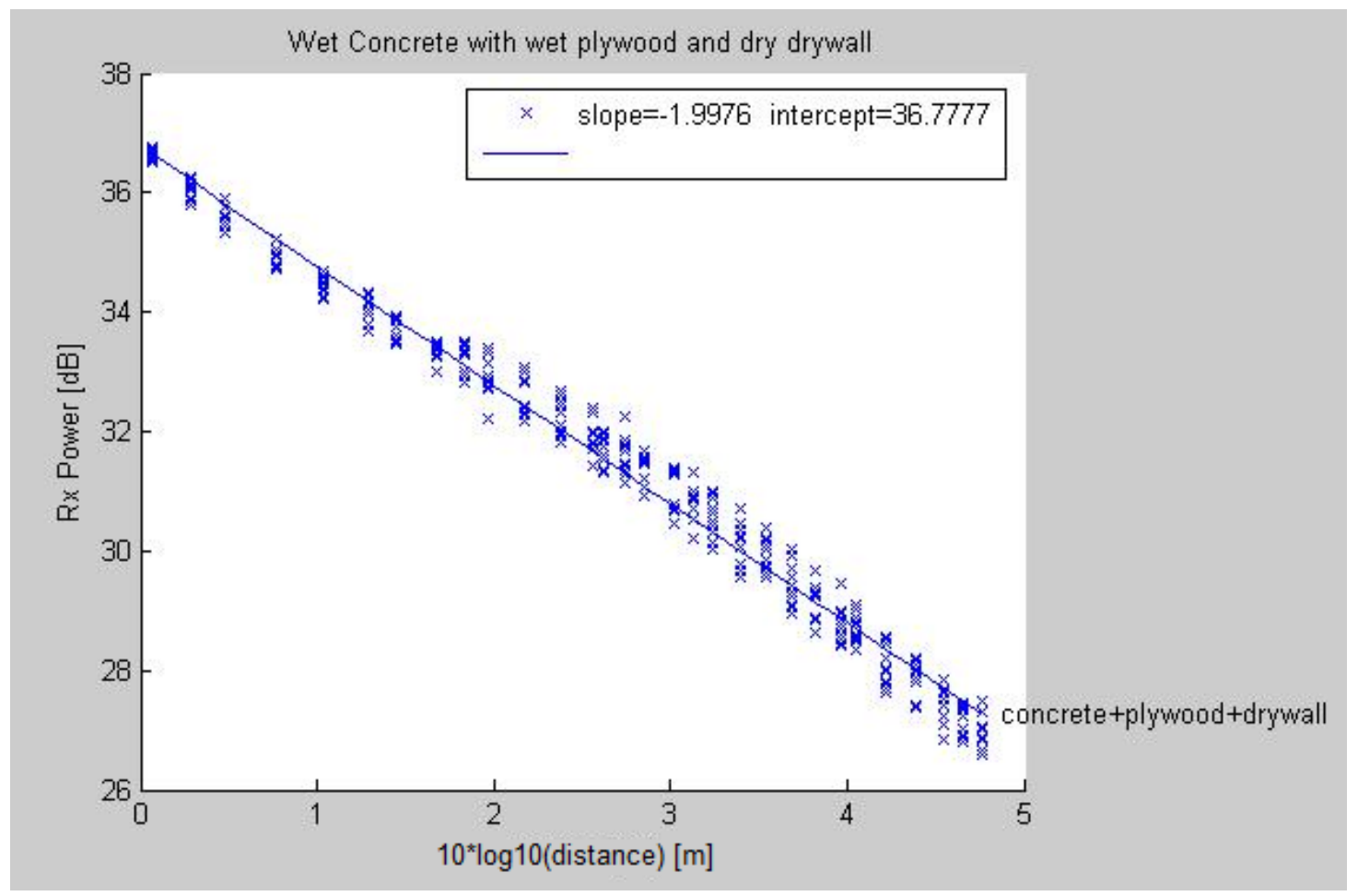

Figure 5.17. Wet concrete with wet plywood and dry drywall 


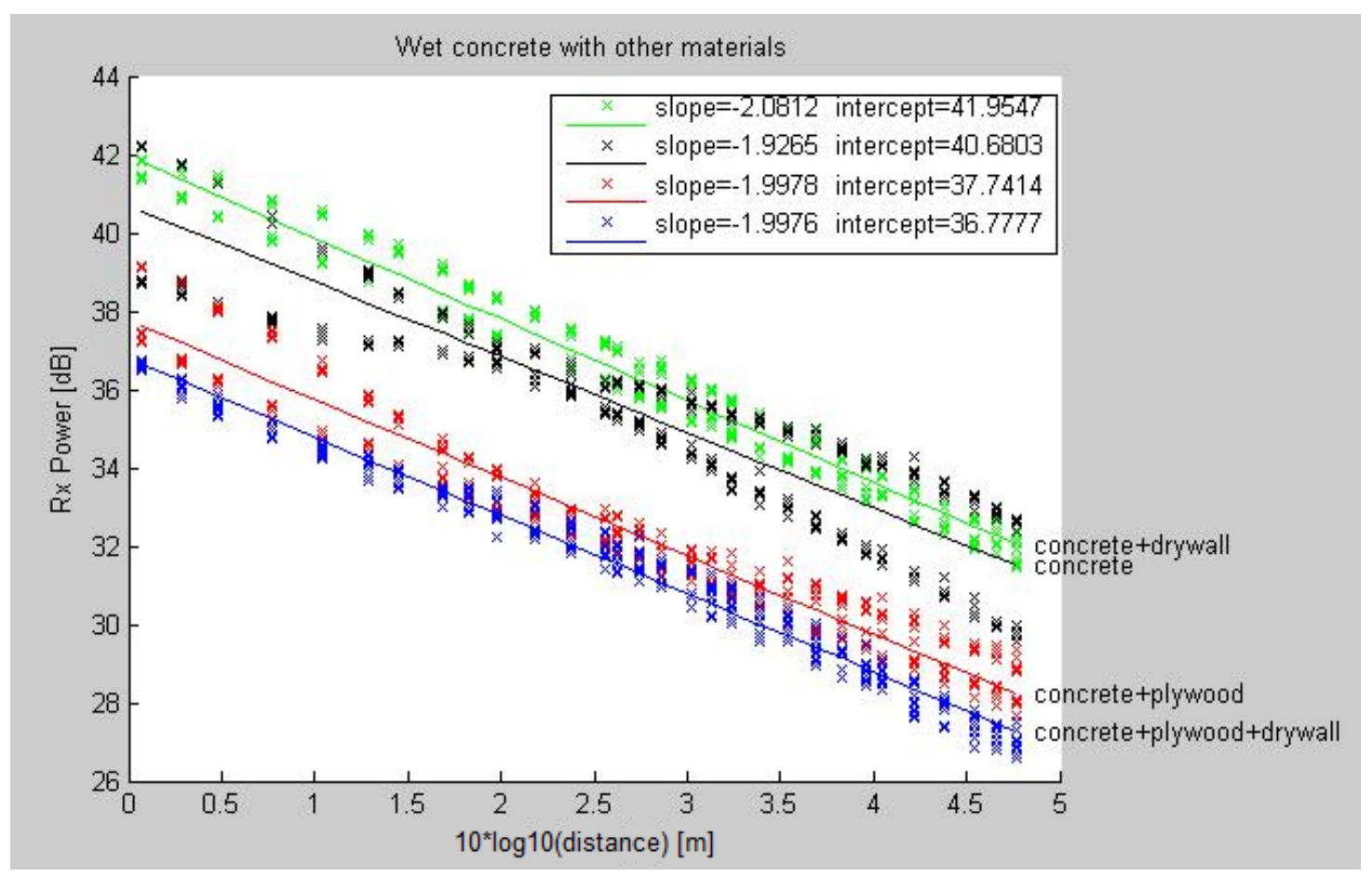

Figure 5.18. Wet concrete with other material

Above plots were put into one plot in Figure 40. Wet concrete result is similar to dry reinforced concrete result, where the signal attenuation is largest when all materials were put together (i.e., option $(d))$.

\subsubsection{When compared to Dry Material Result}

Figure 5.24 shows when comparing wet material result to dry material one. Both wet concrete and wet reinforced concrete has better received signal power compared to dry concrete and dry reinforced concrete. It seems that signal attenuates less when material is wet. 


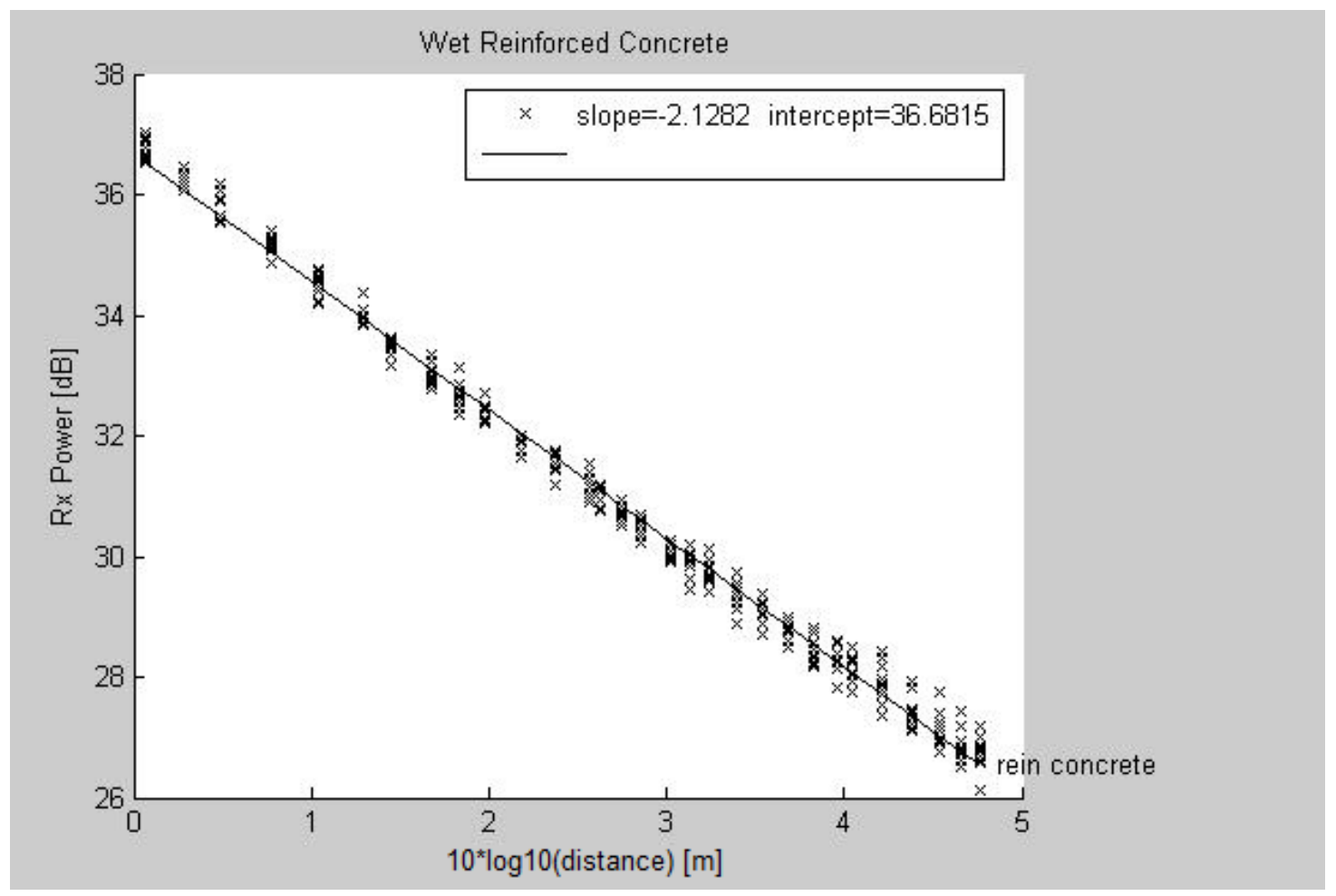

Figure 5.19. Wet reinforced concrete pathloss 


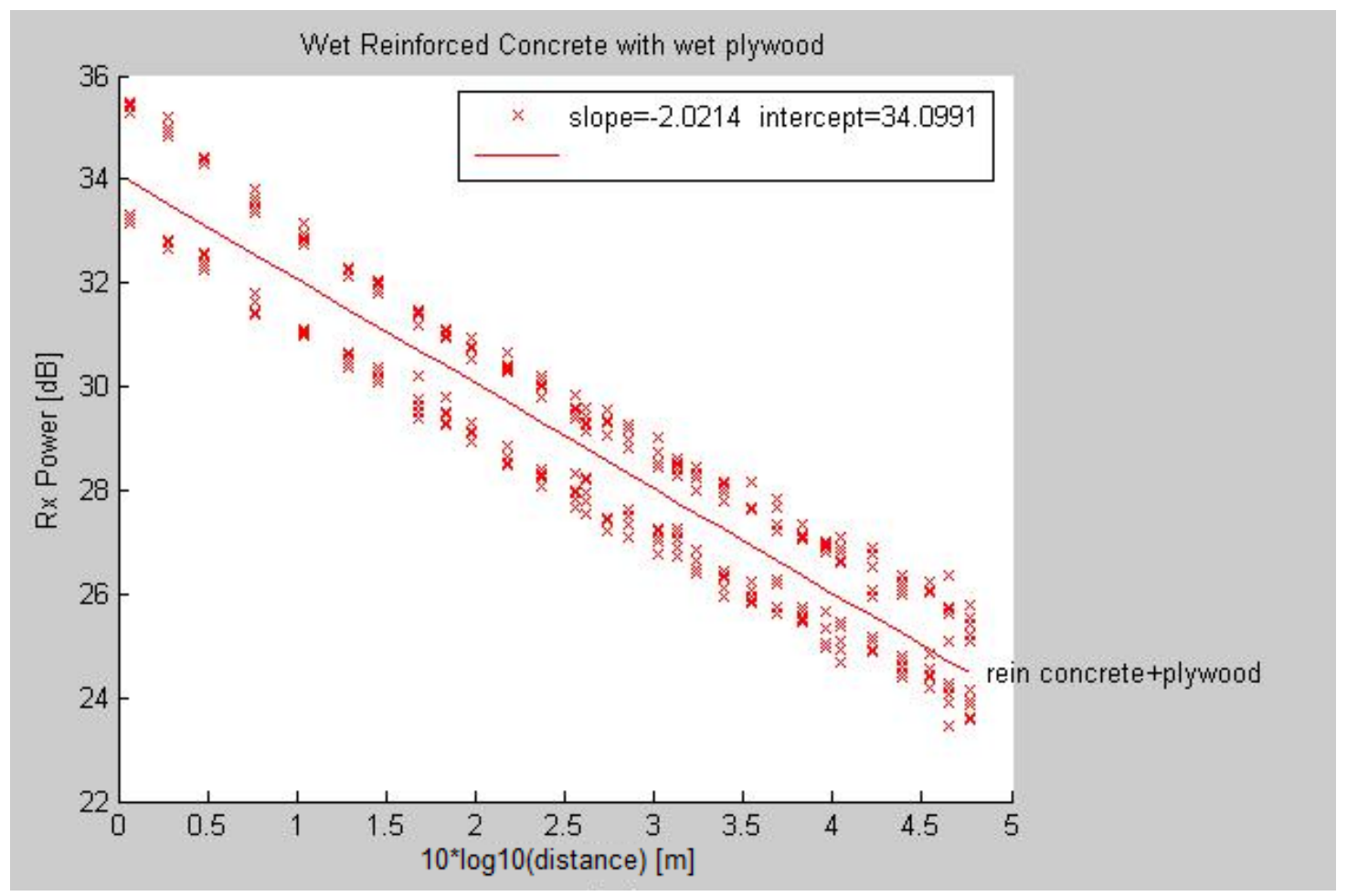

Figure 5.20. Wet reinforced concrete with wet plywood 


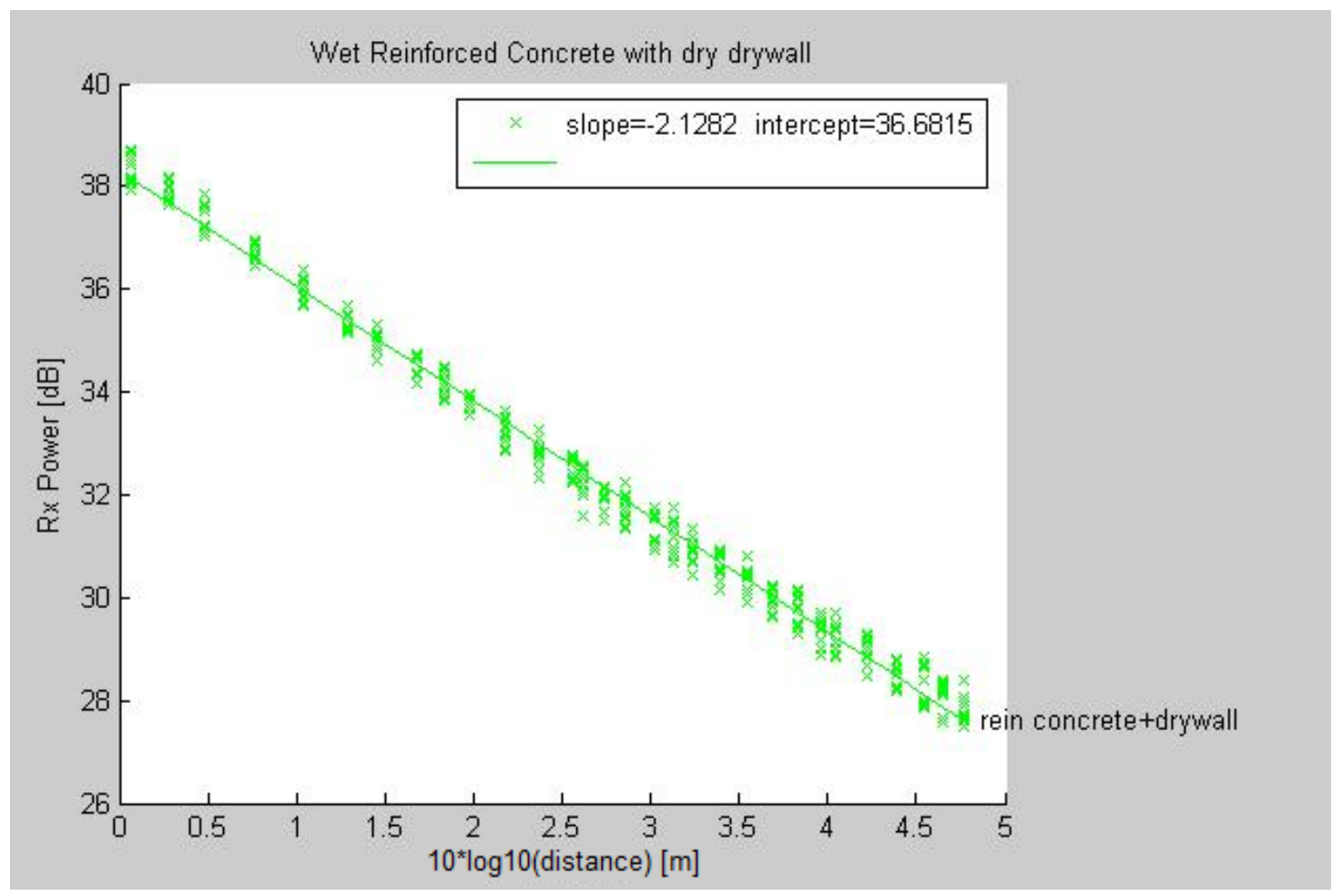

Figure 5.21. Wet reinforced concrete with dry drywall 


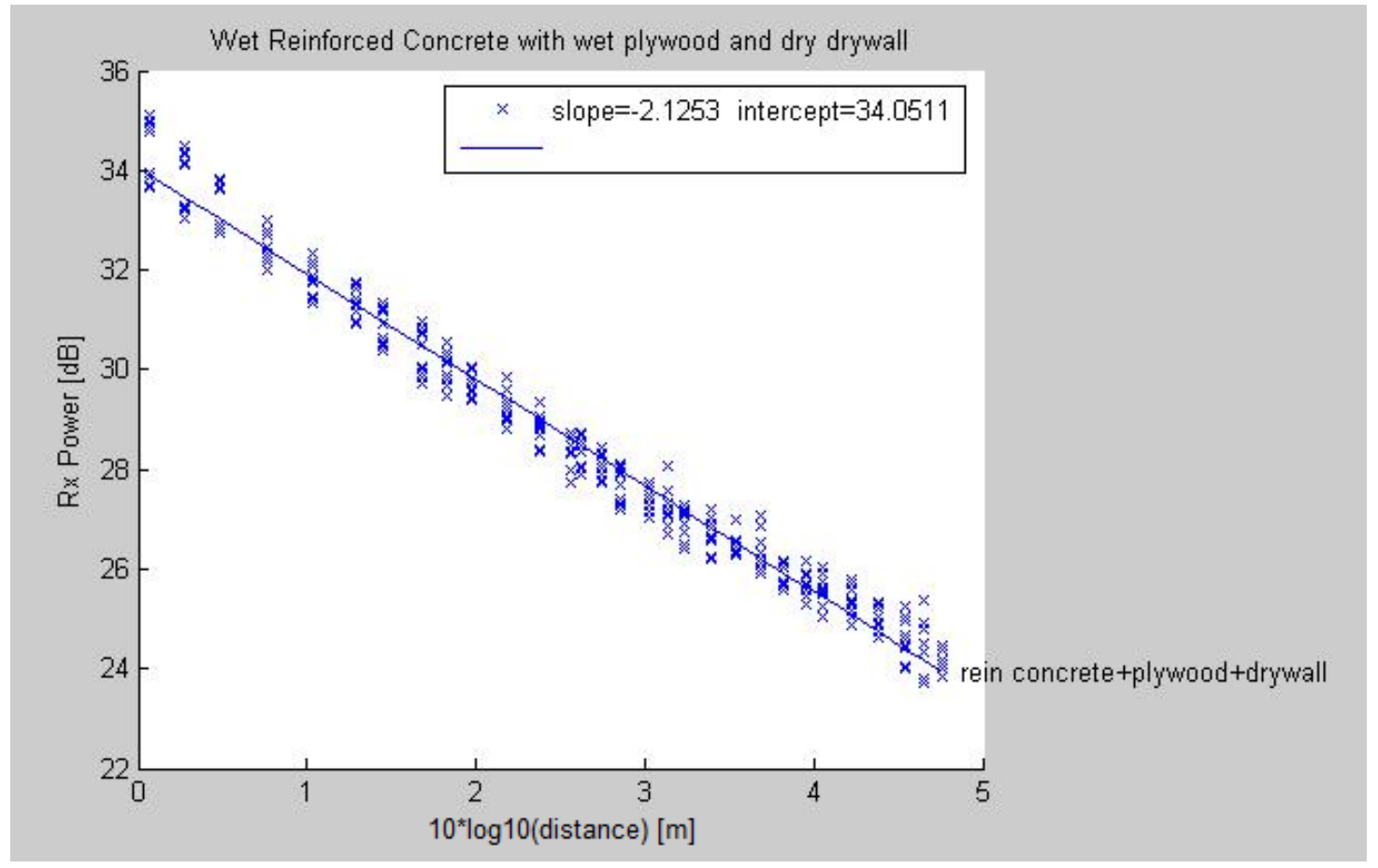

Figure 5.22. Wet reinforced concrete with wet plywood and dry drywall

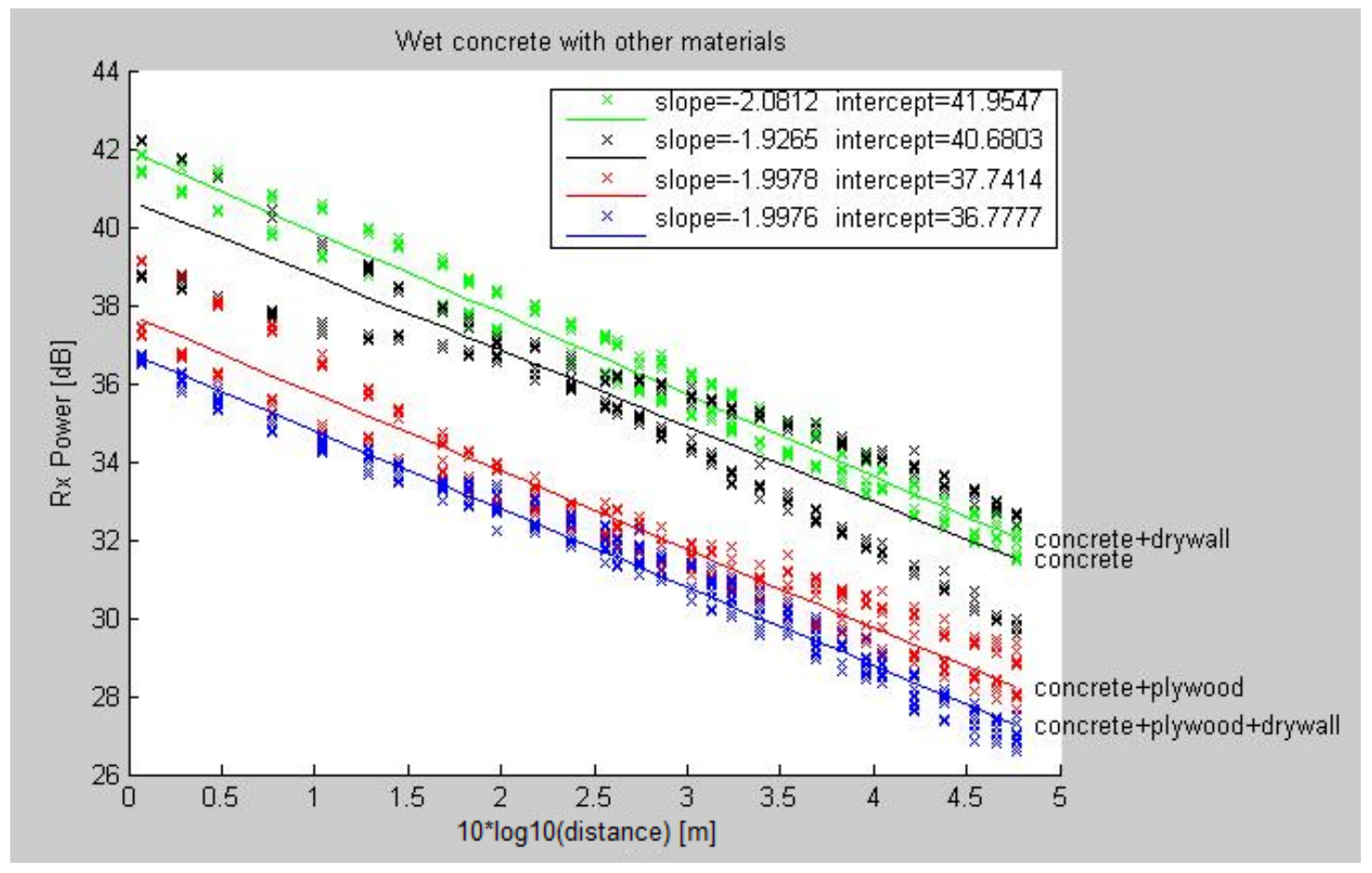

Figure 5.23. Wet reinforced concrete with other material 


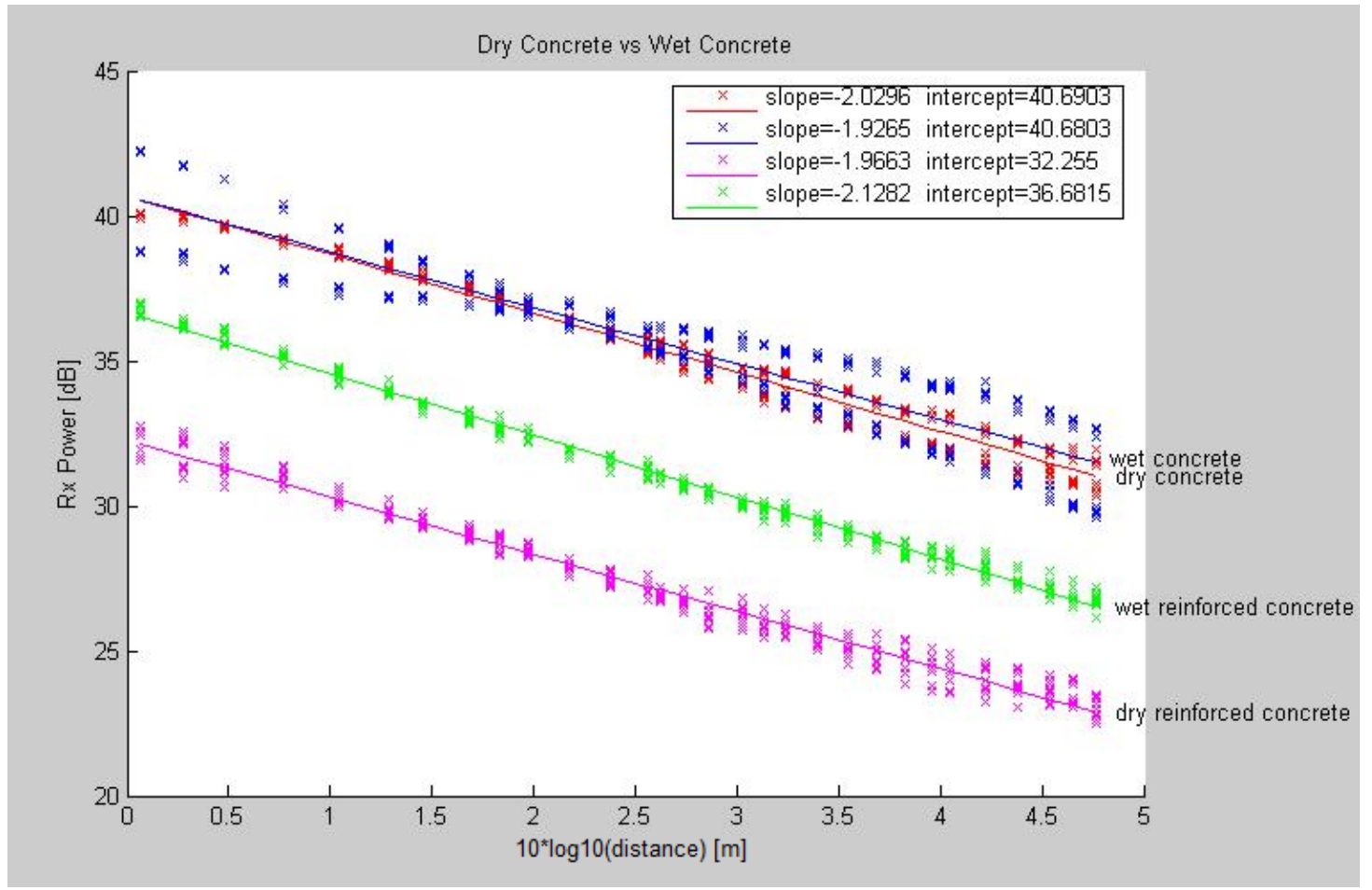

Figure 5.24. Dry versus wet material 
Chapter 6

\section{WALL TYPE AND THICKNESS IDENTIFICATION}

As explained in chapter 2, the focus of this research is to solve the problem of identifying the material type and thickness as well as mapping walls by applying data fusion algorithms on transmitted pulses between radios, i.e., problem of material sensing and wall mapping. This chapter presents the details of the first sensing

problem, which is wall type and thickness identification. Details on wall mapping will be presented in the next chapter. The remainder of this chapter first explains the research challenges, wall identification algorithm, and methodology, and then presents results of applying the algorithm for wall type and identification.

\subsection{RESEARCH CHALLENGE FOR WALL TYPE AND THICK- NESS IDENTIFICATION}

In order to understand the challenge of determining wall composition of cavities, it is instructive to consider Figure 6.1. Here we see two radios $A$ and $B$ in different cavities that are separated by a wall. We need to determine the thickness $d_{w}$ as well as the type of material comprising the wall.

This approach requires an accurate distance measurement between the two radios. UWB has a very large bandwidth and therefore has high accuracy in determining ToA in free space. However, in complex indoor environment, as radio 


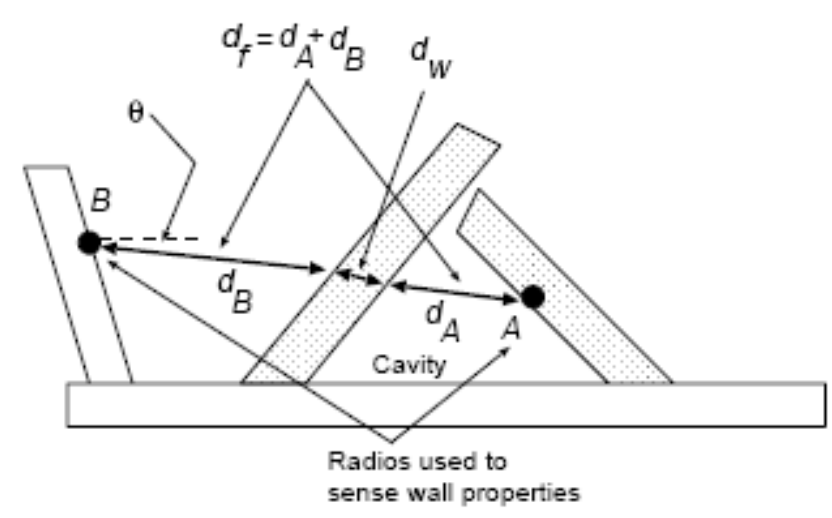

Figure 6.1. Radio locationing and material sensing example

signals propagate, they suffer attenuation due to distance as well as due to absorption by intervening materials. There is error due to multipath effects, where a reflection can be mistaken for the LOS signal, as well as error due to weak received signals (which require longer integration periods for detection). Due to such errors the value of $d_{f}$ in Figure 6.1 can be inaccurate resulting in incorrect material sensing. In order to understand the error as well as develop ways to mitigate the effects of error, we performed extensive measurements of UWB signal propagation through materials, and also developed an algorithm that can accurately determine wall type and thickness under the adverse multipath effect.

\subsection{ALGORITHM FOR WALL TYPE AND THICKNESS IDENTI- FICATION}

This section explains the algorithm we developed for wall type and thickness identification using signal propagation measurements between two radios. Our approach used the Received Signal Strength (RSS) of a transmission from $A$ at $B$ (Figure 6.1) to determine the wall's properties. The three values - distance between the two radios, $d_{w}$, and the wall type - will be estimated simultaneously 
using path loss characteristics of the walls and RSS. The algorithm to determine the wall type and thickness of the middle wall in Figure 6.1 is as follows:

1. Let us assume that the wall thickness is $d_{w}$ and the sum of the two distances between each radio and the wall is $d_{f}$ (freespace distance). Thus, the distance between the two radios $A$ and $B$ is $y=d_{f}+d_{w}$.

2. Let radio $A$ be the transmitter and radio $B$ the receiver. Radio $A$ transmits a short pulse (nanosecond in length) to $B$. Radio $B$ returns the pulse back to $A$ at a pre-determined power. We assume that radio $A$ either has two receivers or two antennas separated by some distance, where they form a straight line with the antenna of $B$. The two values of the received power at $A$ are $p_{1}$ and $p_{2}$. Let the time difference of when each antenna at $A$ receives the signal be $\delta_{t}$ and we set $x=c \delta_{t} \mathrm{~m}$, where $x$ indicates the difference in distances of the two antennas or receivers from the transmitter, and $c$ is the speed of light in freespace. Let us arbitrarily assume that the signal is received at antenna 1 first (i.e., it is closer to $B$ ).

3. Generally the material used for construction is known apriori. Indeed, the main load-bearing members used typically have well-defined thicknesses and composition (e.g., 8 inch concrete etc.). Assume that we characterize the path loss seen between two radios separated by some distance $D$ and with one piece of building material (of various thicknesses) in between. We can plot the received signal strength (for a given transmit power) versus $D$ for different materials as shown in Figure 6.2.

4. The distance $y_{1}$ (i.e., distance to closer receiver) is an unknown. However, we know that at distance $y_{1}$ the received power is $p_{1}$ and at $y_{2}=y_{1}+x$ it is $p_{2}$. We draw a horizontal line segment in Figure 6.2 corresponding to 
power levels $p_{1}$ and $p_{2}$. We guess a value for $y_{1}$ (call it $\overline{y_{1}}$ ) and construct a line segment connecting points $\left(\overline{y_{1}}, p_{1}\right)$ and $\left(\overline{y_{2}}, p_{2}\right)$. We compute the Mean Square Error (MSE) between this line segment and the pathloss line for each material. The range of values for $\overline{y_{1}}$ goes from zero to $y_{\max }$ where $y_{\max }$ is determined using time of arrival (ToA) data from the UWB radio. Assuming freespace, the ToA information gives us an upper bound on $\overline{y_{1}}$ (if material is present then $\overline{y_{1}}$ is smaller since speed of the signal is less in material) and this is the value used for $y_{\max }$.

5. Among all the potential fits, we select the one that minimizes the MSE. This gives us a good estimate for $y_{1}$ as well the material and the thickness (read off from the path loss curves).

\subsection{WALL TYPE AND THICKNESS IDENTIFICATION METHOD- OLOGY}

In order to verify that the algorithm to determine the type and thickness of material works, a series of very detailed signal propagation measurements were conducted. There were two main steps in this experimental work.

1. Characterizing the pathloss for a variety of building material in order to produce a graph like Figure 6.2.

2. Run experiments to identify the type and thickness of the wall as described in the algorithm.

\subsubsection{Applying Fingerprinting Algorithm}

Figure 6.3 shows reference measurements of signal propagation through different types of material. It plots the pathloss for freespace, reinforced concrete (7.5 in), 


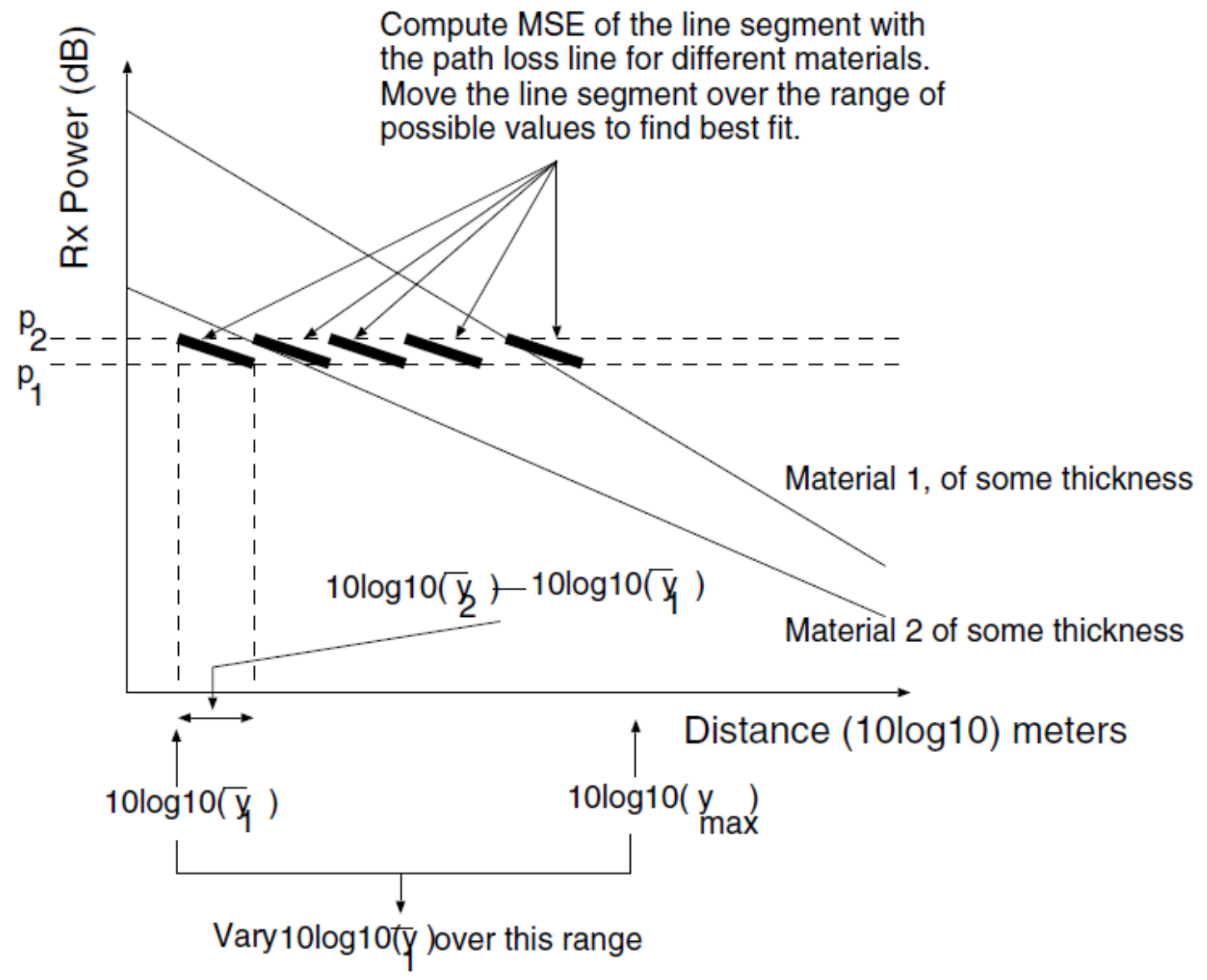

Figure 6.2. Illustration of the algorithm 


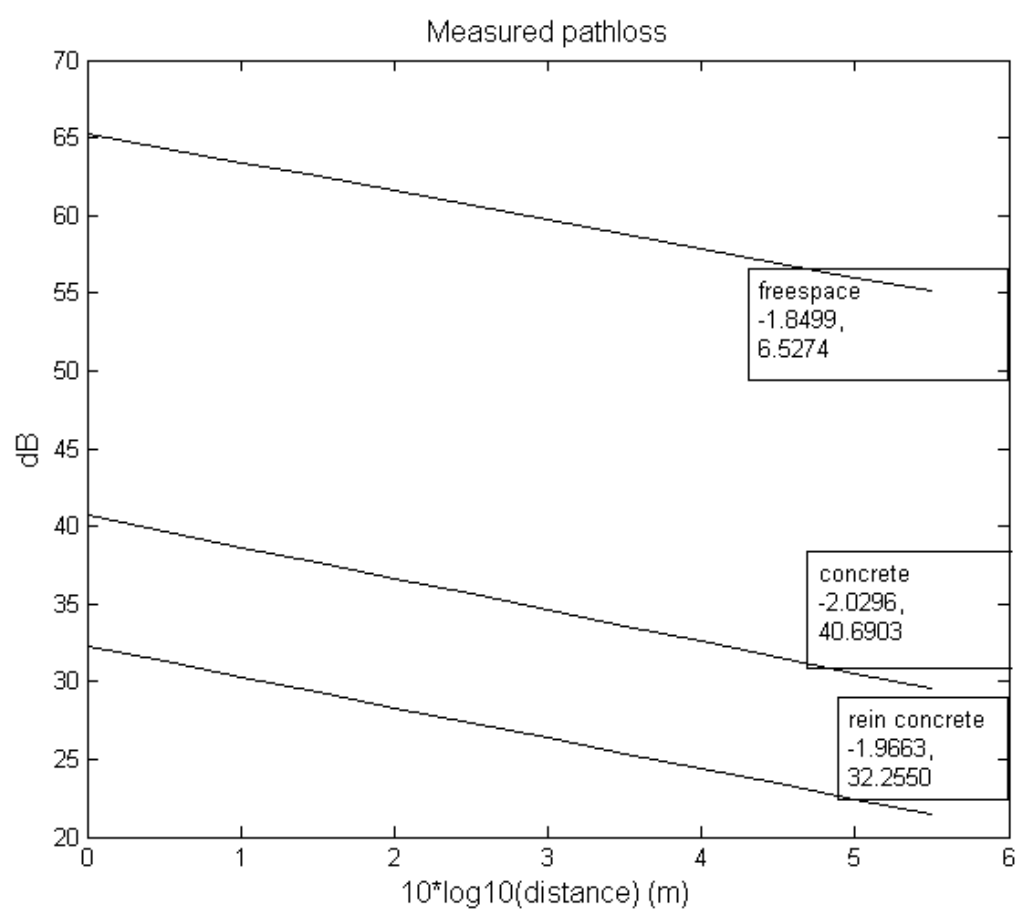

Figure 6.3. Pathloss for a subset of dry material

and concrete $(6.5 \mathrm{in})$. The numbers next to each line indicate the slope and the $y$-intercept. For clarity we have left out the individual measurements and only retained the best fit lines. As we can see, the curves are well separated and therefore identifying the type of wall using our algorithm is feasible.

In order to test out algorithm, the following experimental methodology was used for each material or combination of material:

- We used two receivers and one transmitter. Let $y_{1}$ and $y_{2}$ denote the distance of the two antennas or receivers from the transmitter.

- We varied $y_{1}$ between 1 and 3 meters (to better represent small cavities found in fallen buildings). $y_{2}$ also varied between these values but was greater than $y_{1}$ because $y_{1}$ is the distance to closer receiver or antenna. In all there were 
450 pairwise measurement points. For each $y_{1}$ and $y_{2}$ value, we collected 5 measurements of received power $p$ and ToA per receiver that was total of 5 x 5 . Thus, we collected a total of $450 \times 5 \times 5$ data points.

- For each pair of $p_{1}$ and $p_{2}$ values, we ran the algorithm described in section 6.2 and found:

- Type of material and thickness (the answer is a 1 or 0 depending on whether we guessed correctly or incorrectly).

- The estimated value of $y_{1}$ (since this is also unknown in reality).

In the next section, we present algorithm application results in detail by calculating the percentages of the correct guesses between the predicted values and the actual values.

\subsection{RESULTS FOR WALL TYPE AND THICKNESS IDENTIFICA- TION}

This section shows result of applying our material identification algorithm for wall type and thickness. The method of applying our algorithm was explained in section 6.3.1, and the reference pathloss plot is shown in Figure 6.3.

Table 6.1 summarizes the main results for three materials. As we can see, the algorithm correctly determines the material type and thickness almost all the time. Plywood was the only material where the algorithm made some mistakes. This is due to the fact that plywood does not attenuate the signal much as compared to concrete or reinforced concrete and thus the algorithm sometimes concludes freespace as the material rather than wood. The table also summarizes the error in estimating $y_{1}$. Notably, plywood has the highest error while for reinforced 
concrete the error is negligible. Indeed, in the case of plywood, the algorithm always underestimates the value of $y_{1}$.

Table 6.1. Summary of algorithm performance

\begin{tabular}{|l|l|l|}
\hline Material & $\begin{array}{l}\text { Material thickness \& type } \\
\text { guess }\end{array}$ & $\begin{array}{l}\text { Mean absolute error in } y_{1} \\
\text { estimate }\end{array}$ \\
\hline Freespace & $100 \%$ & $4.84 \%$ \\
\hline Plywood & $98.7 \%$ & $15.7 \%$ \\
\hline Concrete & $99.39 \%$ & $3.39 \%$ \\
\hline $\begin{array}{l}\text { Reinforced } \\
\text { Concrete }\end{array}$ & $99.95 \%$ & $0.76 \%$ \\
\hline
\end{tabular}

In order to understand the error in distance estimate, we plot the error (expressed as a percentage) in estimating $y_{1}$ versus $y_{2}-y_{1}$ (i.e, the distance between the two receivers). Figure 6.4, Figure 6.5, Figure 6.6 and Figure 6.7 show the plot for freespace, plywood, concrete, and reinforced concrete in that order. The error for freespace is highly variable for all $y_{1}-y_{2}$ values and is explained by the severe multipath present in the laboratory which is essentially a large concrete sub-basement used for testing concrete structures. Interestingly, the error is small and consistent for reinforced concrete primarily because a great deal of signal energy is absorbed by the reinforced concrete leaving little energy in various reflected components. In view of our original application, this is good news since we can conclude that in the real world our algorithm ought to provide good estimates. 


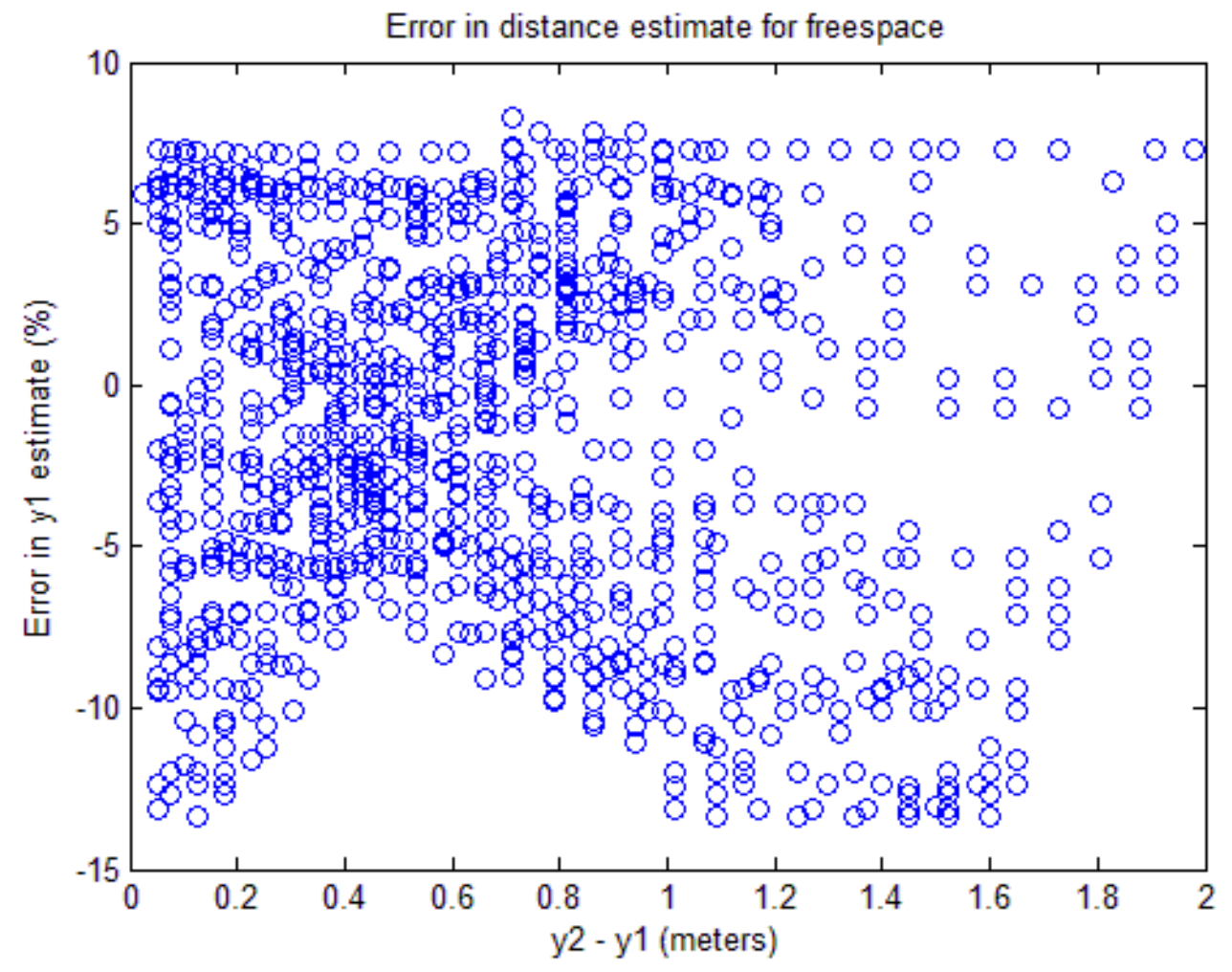

Figure 6.4. Error in distance estimate for freespace 


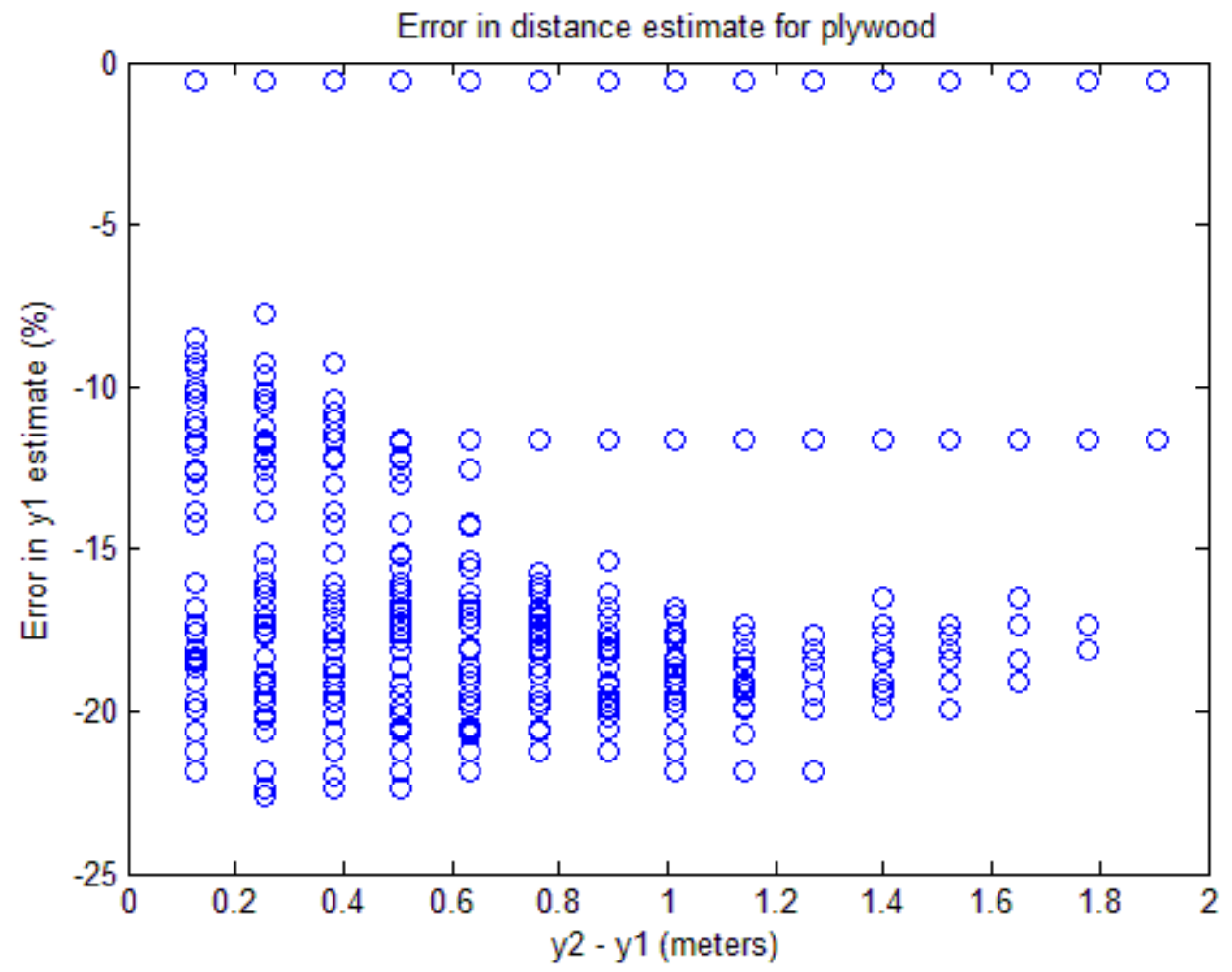

Figure 6.5. Error in distance estimate for plywood 


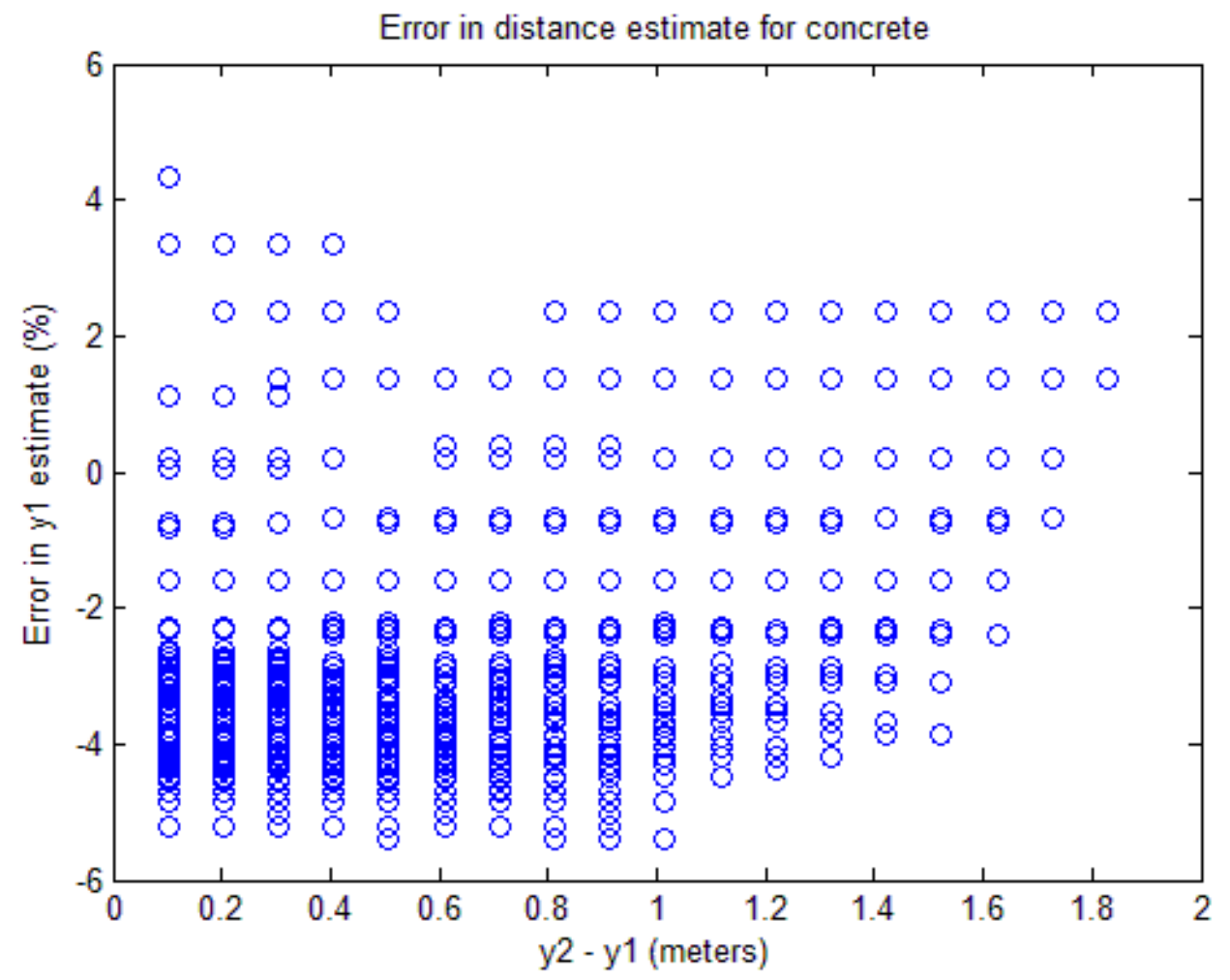

Figure 6.6. Error in distance estimate for concrete 


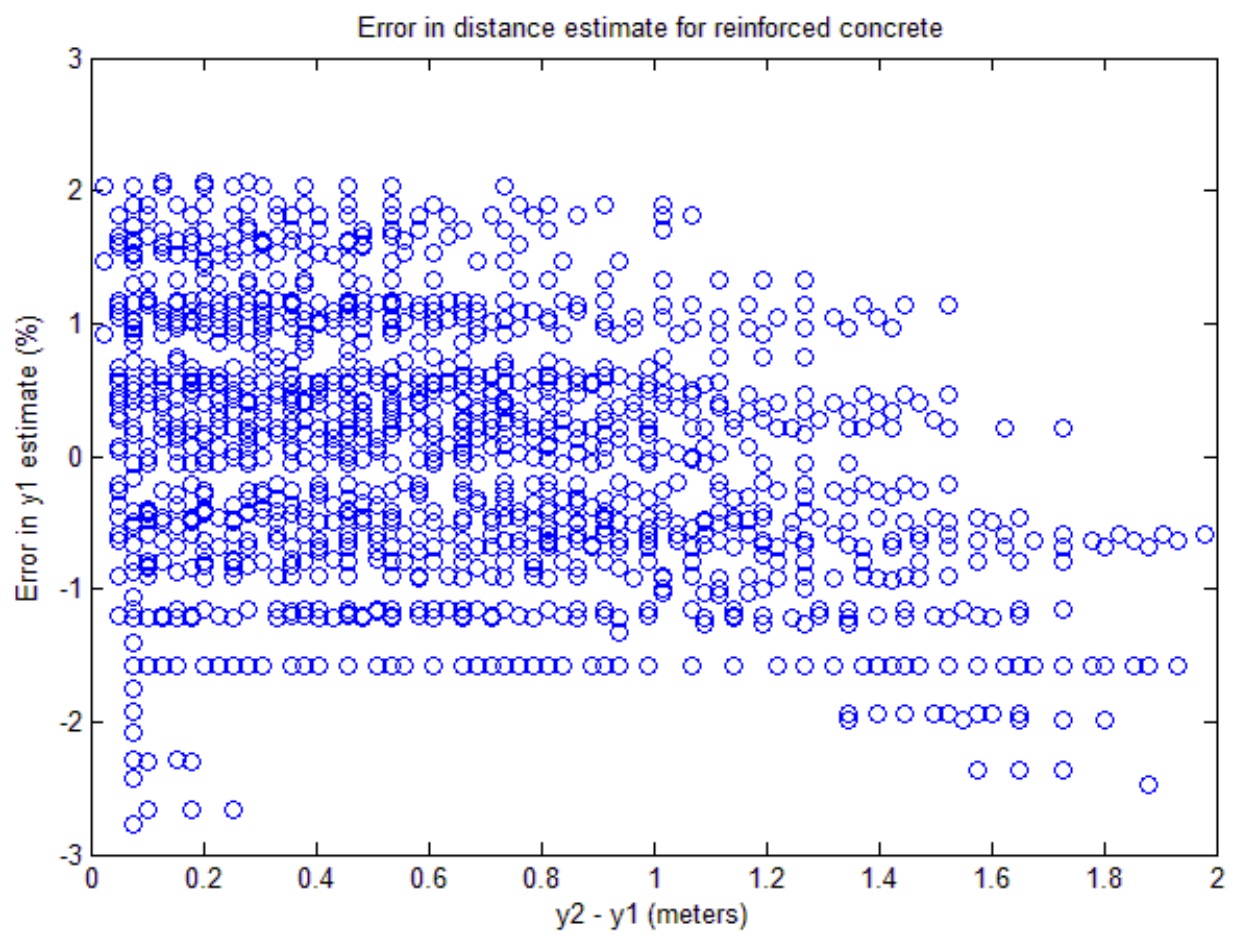

Figure 6.7. Error in distance estimate for reinforced concrete 


\section{Chapter 7}

\section{D WALL MAPPING}

In this chapter, we discuss the algorighm to estimate the position of the wall by locationing radios in 3D space and present the result of wall mapping measurements and wall position estimation. In order to estimate radio position in a small cavity using ToA and AoA, we performed extensive in-lab measurement of ToA and AoA in various configurations and angles using omni-directional transmission. Wall orientation was also varied to understand the impact of multipath on estimation errors. Experiments were done for LOS case where two radios were both in the same cavity. From the measured set of AoAs from the baseline and the actual AoA value we know, database entries were created with the tuple. Same applied to the ToA database. This became the "fingerprint" for locationing. This measurement database was used to characterize the positioning accuracy. We used a geometric technique to estimate the position of radios. Measurement values were significantly affected when multipath effect was severe. In such case, positioning accuracy was decreased.

\subsection{ALGORITHM FOR RADIO POSITION ESTIMATION}

This section first explains radio position estimation algorithm in 2D space and then explains how it is extended for 3D space radio position estimation. Section 7.1.1 illustrates 2D radio position estimation algorithm, and section 7.1.2 shows $3 \mathrm{D}$ radio position estimation algorithm used in this research. 


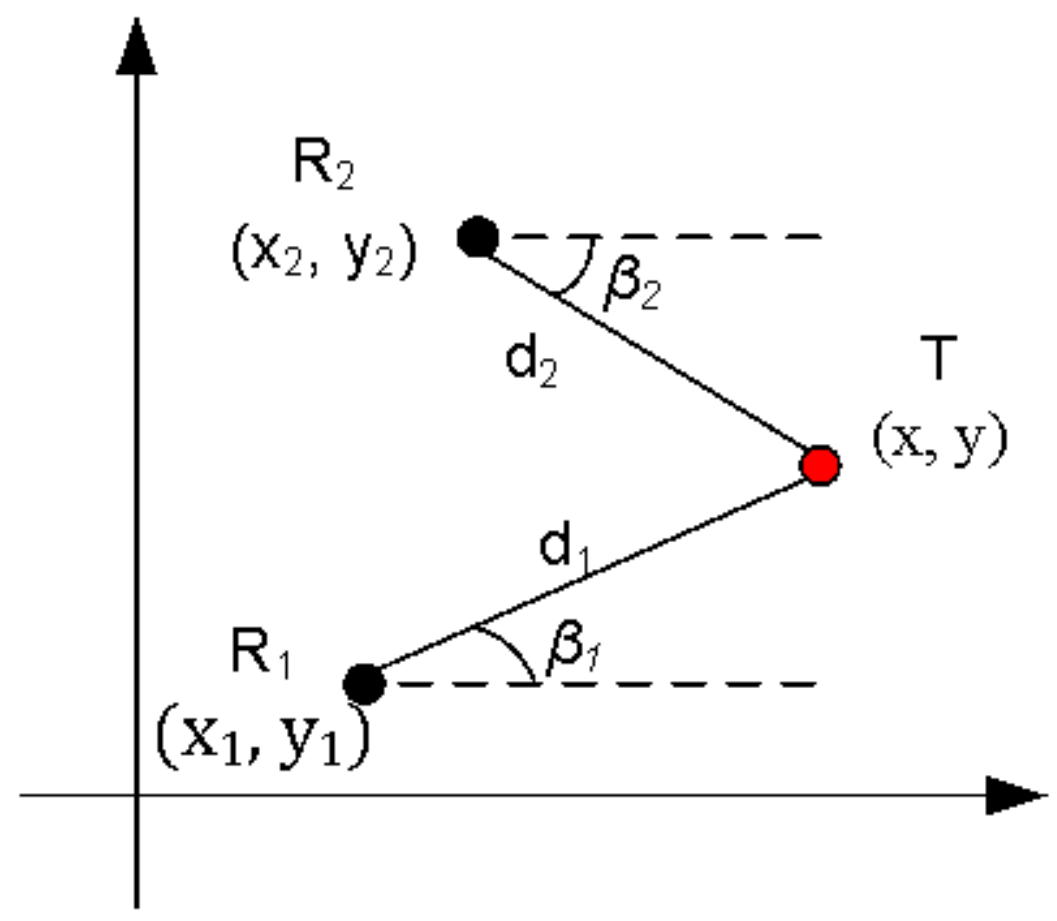

Figure 7.1. Position of target node in 2D space

\subsubsection{D Radio Position Estimation Algorithm}

When the $R_{i}$ in Figure 7.1 is the $i$ th reference node and the $T$ is the target node, the position estimation of $T$ from the reference node $R_{i}$ is expressed as:

$$
\begin{aligned}
& \hat{x}_{i}=x_{i}+d_{i} \cos \beta_{i} \\
& \hat{y}_{i}=y_{i}+d_{i} \sin \beta_{i},
\end{aligned}
$$

where $\left(\hat{x}_{i}, \hat{y}_{i}\right)$ is the estimation of position $T$ from the $i$ th reference node, $R_{i}$, $\left(x_{i}, y_{i}\right)$ is the position of the reference node $R_{i}, d_{i}$ is the distance between the $R_{i}$ and the $T$, and $\beta_{i}$ is the AoA of the signal from $T$ to $R_{i}$. Section 8.2 provides further details on traditional indoor positioning algorithms using other metrics such as RSS, ToA, TDoA, AoA, RSSI and hybrid schemes. 


\subsubsection{D Radio Position Estimation Algorithm}

This section explains the 3D target position estimation algorithm used in our research, which utilizes ToA based location sensing. Location sensing can be done by measuring RSS, AoA and/or ToA as location metrics. However, due to severe multipath propagation and heavy shadow fading in indoor environment, the measurements of RSS and AoA provide less measurement accuracy than does ToA [72]. This can be seen in our AoA measurement results in this chapter, which provide high measurement error compared to ToA based distance measurements. Thus, we developed the target position estimation algorithm using ToA based distance measurement. ToA method measures the distance between the reference node and the target node by estimating signal propagation delay between signal transmission time and reception time.

Let $\left(x_{i}, y_{i}, z_{i}\right)$ in Figure 7.2 be the coordinate of the $i$ th reference node, $R_{i}$, and $(x, y, z)$ the coordinate of the target node, $T$, then the distance, $d_{i}$, between $R_{i}$, and $T$, is:

$$
d_{i}=\sqrt{\left(x-x_{i}\right)^{2}+\left(y-y_{i}\right)^{2}+\left(z-z_{i}\right)^{2}}
$$

Since the position of any reference nodes, $\left(x_{i}, y_{i}, z_{i}\right), i=1, \ldots, N$, and the distance, $d_{i}$, are known values from the rest of the sections in this chapter, the estimated position of the target node $T,(\hat{x}, \hat{y}, \hat{z})$, can be obtained by selecting

any three distances from three reference nodes as (7.4) - (7.6) and calculating the target position [71][63].

$$
\begin{aligned}
& d_{i}=\sqrt{\left(\hat{x}-x_{i}\right)^{2}+\left(\hat{y}-y_{i}\right)^{2}+\left(\hat{z}-z_{i}\right)^{2}} \\
& d_{j}=\sqrt{\left(\hat{x}-x_{j}\right)^{2}+\left(\hat{y}-y_{j}\right)^{2}+\left(\hat{z}-z_{j}\right)^{2}}
\end{aligned}
$$




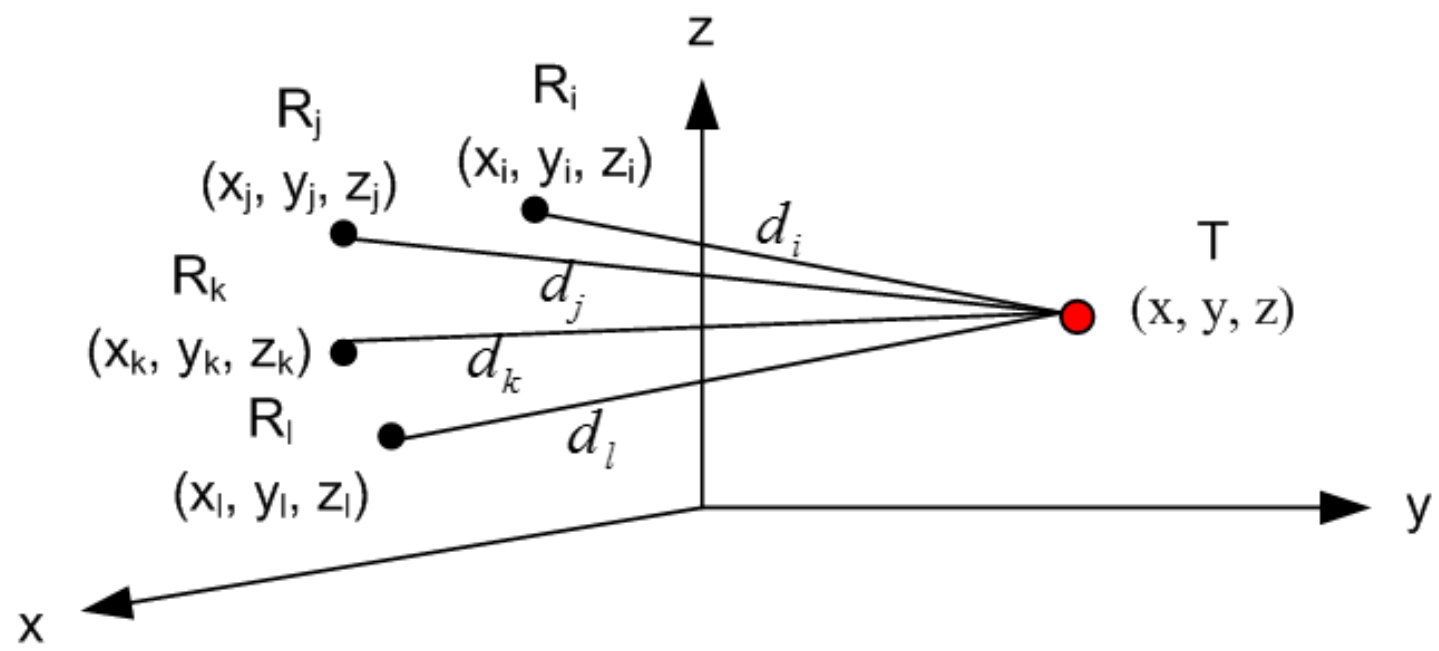

Figure 7.2. Position of target node in 3D space

$$
d_{k}=\sqrt{\left(\hat{x}-x_{k}\right)^{2}+\left(\hat{y}-y_{k}\right)^{2}+\left(\hat{z}-z_{k}\right)^{2}}
$$

These equations can be transformed to a system of linear equations by squaring them first and then subtracting one of the equations from each of the others [71][63][74]. For example, by subtracting $d_{j}^{2}$ and $d_{k}^{2}$ from $d_{i}^{2}$, and $d_{k}^{2}$ from $d_{j}^{2}$, we obtain (7.7) - (7.9) without non-linear terms $\hat{x}^{2}, \hat{y}^{2}$, and $\hat{z}^{2}$.

$$
\begin{aligned}
& d_{i}^{2}-d_{j}^{2}=2\left(x_{j}-x_{i}\right) \hat{x}+2\left(y_{j}-y_{i}\right) \hat{y}+2\left(z_{j}-z_{i}\right) \hat{z} \\
& +x_{i}^{2}+y_{i}^{2}+z_{i}^{2}-x_{j}^{2}-y_{j}^{2}-z_{j}^{2} \\
& d_{i}^{2}-d_{k}^{2}=2\left(x_{k}-x_{i}\right) \hat{x}+2\left(y_{k}-y_{i}\right) \hat{y}+2\left(z_{k}-z_{i}\right) \hat{z} \\
& +x_{i}^{2}+y_{i}^{2}+z_{i}^{2}-x_{k}^{2}-y_{k}^{2}-z_{k}^{2} \\
& d_{j}^{2}-d_{k}^{2}=2\left(x_{k}-x_{j}\right) \hat{x}+2\left(y_{k}-y_{j}\right) \hat{y}+2\left(z_{k}-z_{j}\right) \hat{z} \\
& +x_{j}^{2}+y_{j}^{2}+z_{j}^{2}-x_{k}^{2}-y_{k}^{2}-z_{k}^{2}
\end{aligned}
$$


These equations (7.7) - (7.9) can be expressed as matrix form:

$$
A \hat{X}=b
$$

where

$$
\begin{gathered}
A \triangleq\left[\begin{array}{lll}
2\left(x_{i}-x_{j}\right) & \left(2\left(y_{i}-y_{j}\right)\right. & 2\left(z_{i}-z_{j}\right) \\
2\left(x_{i}-x_{k}\right) & \left(2\left(y_{i}-y_{k}\right)\right. & 2\left(z_{i}-z_{k}\right) \\
2\left(x_{j}-x_{k}\right) & \left(2\left(y_{j}-y_{k}\right)\right. & 2\left(z_{j}-z_{k}\right)
\end{array}\right] \\
b \triangleq\left[\begin{array}{l}
x_{i}^{2}+y_{i}^{2}+z_{i}^{2}-x_{j}^{2}-y_{j}^{2}-z_{j}^{2}-d_{i}^{2}+d_{j}^{2} \\
x_{i}^{2}+y_{i}^{2}+z_{i}^{2}-x_{k}^{2}-y_{k}^{2}-z_{k}^{2}-d_{i}^{2}+d_{k}^{2} \\
x_{j}^{2}+y_{j}^{2}+z_{j}^{2}-x_{k}^{2}-y_{k}^{2}-z_{k}^{2}-d_{j}^{2}+d_{k}^{2}
\end{array}\right],
\end{gathered}
$$

and

$$
\hat{X} \triangleq\left[\begin{array}{l}
\hat{x} \\
\hat{y} \\
\hat{z}
\end{array}\right]
$$

Then, the position of the target node $\hat{X}$ is estimated by solving the matrix as (7.14) only if we have the perfect distance estimates.

$$
\hat{X}=A^{-1} b
$$

However, since the distance estimates $d_{i}, i=1, \ldots, N$, are imperfect measurements, we can represent the estimated position of the target node with the least square solution [74]:

$$
\hat{X}=\left(A^{T} A\right)^{-1} A^{T} b .
$$


Once the target node position is estimated from a set of three reference nodes, we repeat the position estimation for $T$ from all combination of three reference nodes, which produces multiple position estimations for the target node $T$. Then, we calculate Euclidean distance between the actual target node position and the estimated position. Next, the mean square error (MSE) of target position estimation error was calculated to understand the estimation performance and choose the estimation with the minimum MSE. 


\subsection{D WALL MAPPING METHODOLOGY}

The fixed and variable experimental parameters for the wall mapping research include:

- Fixed Parameters

- Cavity size: W381 x D335 x H231cm

- Transmit power: $-13 \mathrm{dBm}$

- Number of antennas per radio:

* Sensor 1: 2 radios. One has 2 antennas and the other has 1 antenna.

* Sensor 2: 2 radios with one antenna each.

- Variable Parameters

- Sensor 1 locations: 5 locations at A, B, C, D, and E on wall A (refer Figure 7.4 and Figure 7.5)

- Sensor 2 locations: 140 locations

* 70 locations on wall B (refer Figure 7.4)

* 70 locations on Wall C (refer Figure 7.5)

- Number of repetitions: 1

- Full factorial experimental design

* $5 \times 140 \times 1=700$ total data points

- Metrics

- Distance

- Angle of Arrival 


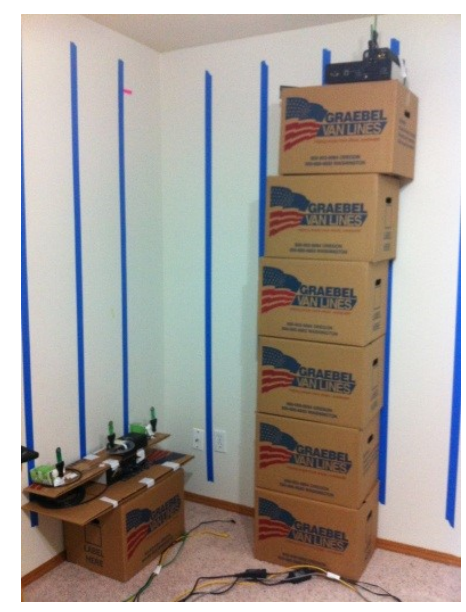

Figure 7.3. 3D wall measurement experimental setup

The experimental setup is shown in Figure 7.3. Sensor 1 is shown on the left and sensor 2 on the right. The PulseOn P200 [90] and the P210 [91] UWB radios from Time Domain Corporation were used for the experiment. In order to simulate a sensor which can measure both Angle of Arrival (AoA) and distance ranging, one P200 radio and one P210 radio were put together for each sensor.

Sensor 1 estimates the AoA [89] and distance [92] of sensor 2. As shown in Figure 3.8, sensor 1 had the P200 AoA receiver and the P210 ranging transmitter, and sensor 2 had the P200 AoA transmitter and P210 ranging receiver. In order to introduce the time difference between UWB reception signals in the antenna array, sensor 1 AoA receiver had two antennas with the antenna separation of 610 $\mathrm{mm}$ (24 inches) and the cable length of $20 \mathrm{ft}$ and 12 inches as shown in Figure 3.8. Antenna separation of 24 inches provides the desired arc accuracy of 3 inches for 0 to $300 \mathrm{~cm}$ distance [89]. The middle antenna of sensor 1 was used to measure the distance ranging. Sensor 2 had two antennas, one for the AoA measurement and the other for the distance ranging. Measurements were done using the sample AoA and Distance measurement applications provided by Time Domain. The flat 


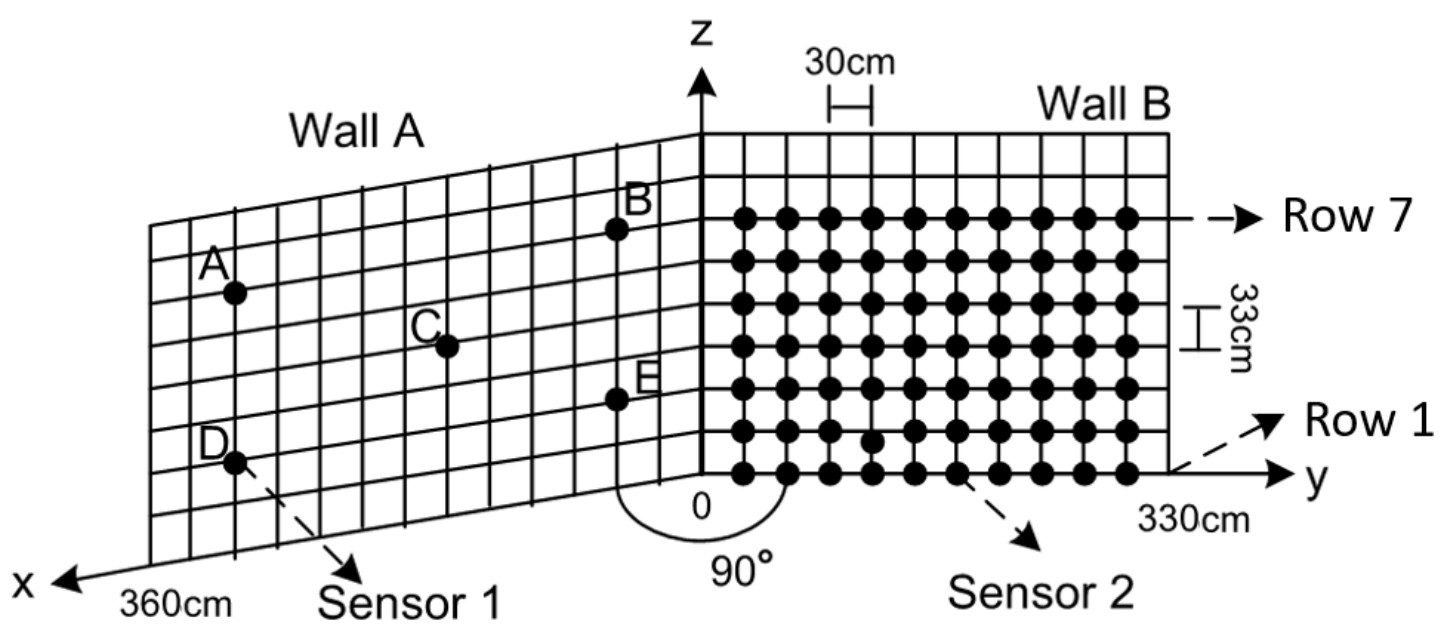

Figure 7.4. Adjoining wall measurement setup

faces of sensor 1 and sensor 2 were placed to be parallel to each other since the received signal is stronger with that configuration [90].

Figure 7.4 shows the adjoining wall experimental setup. Measurements were performed in an empty room with the size of $381 \mathrm{~cm} \times 335 \mathrm{~cm} \times 231 \mathrm{~cm}$ in $x, y, z$ direction. Each wall was marked every $30 \mathrm{~cm}$ horizontally and $33 \mathrm{~cm}$ vertically starting from the $(x, y, z)$ origin. Sensor 1 , which measured the AoA and distance to sensor 2, was placed on the wall A at five different positions, A, B, C, D, and E, and for each sensor 1 position sensor 2 on the wall $\mathrm{B}$ was moved to 10 different columns per seven rows. So, the total of $5 \times 10 \times 7=350$ measurements was done. Row 8 was skipped due to sensors height.

Figure 7.5 shows the opposite wall experiment setup. The experiment was done in the same room as the adjoining wall experiment and each wall was marked the same way, $30 \mathrm{~cm}$ interval horizontally and $33 \mathrm{~cm}$ interval vertically. Sensor 1 was placed on the wall $\mathrm{A}$ at five different positions, $\mathrm{A}, \mathrm{B}, \mathrm{C}, \mathrm{D}$, and $\mathrm{E}$, and for each sensor 1 position sensor 2 on the wall $\mathrm{C}$ was moved to 10 different positions per 


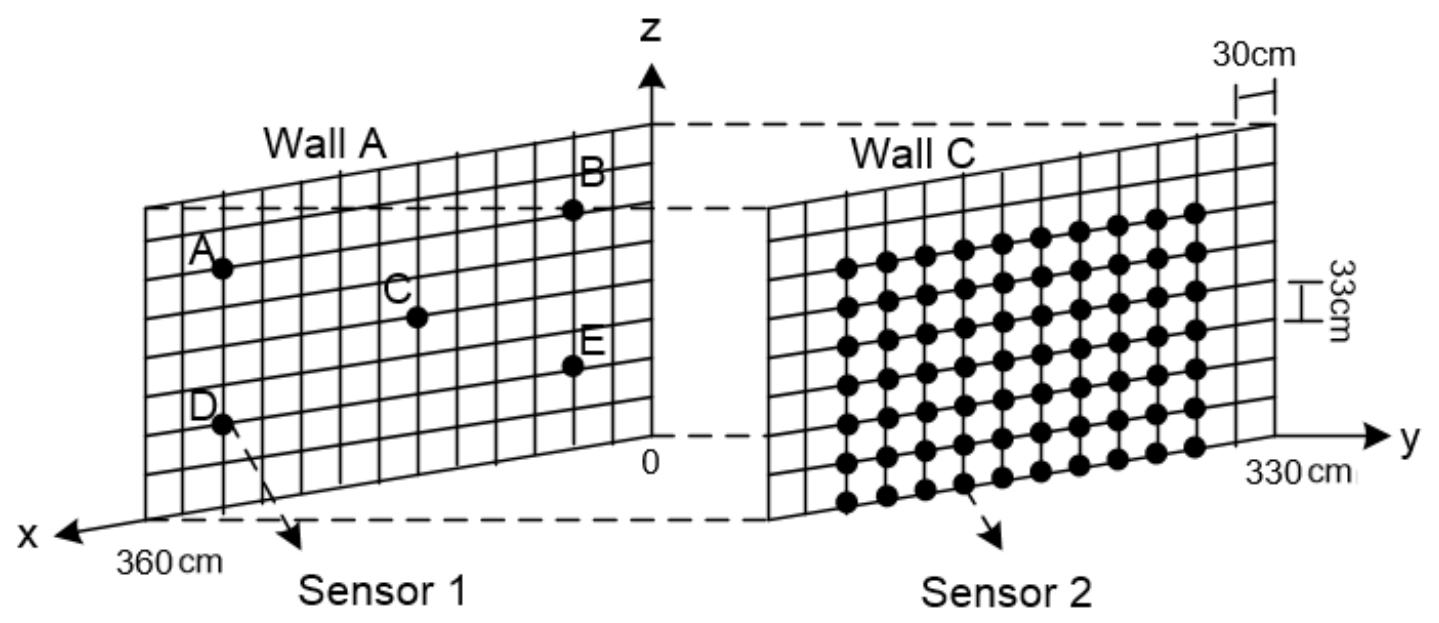

Figure 7.5. Opposite wall measurement setup

seven rows. 


\subsection{RESULTS FOR ADJOINING WALL MEASUREMENT}

Measurement values from distance ranging and AoA experiment for the adjoining walls were analyzed to see the measurement error compared to the actual angle and actual distance.

\subsubsection{Results for Adjoining Wall Distance Measurement}

Figure 7.6, Figure 7.7, and Figure 7.8 show the distance estimation errors compared to actual distance. Figure 7.6 plots distance estimation errors from each sensor 1 position, A, B, C, D, and E, to all sensor 2 positions. More than $95 \%$ of the total errors are less than $4 \%$. High errors occurred when the distance between sensor 1 and sensor 2 was small and sensor 1 was placed near the wall B at position B and E. As sensor 1 moved away from the wall B, the error was reduced and when it was farthest away from the wall $\mathrm{B}$ at position $\mathrm{A}$ and $\mathrm{D}$ the errors were less than $2 \%$. It can be seen more clearly from Figure 7.8, where error on each sensor 1 position was sub-plotted for each sensor 2 row location. The error was greatest when sensor 1 was near wall B at position B and E, medium when sensor 1 was in the middle of wall $\mathrm{A}$ at position $\mathrm{C}$, and smallest when sensor 1 was farthest away from wall $\mathrm{B}$ at position $\mathrm{A}$ and $\mathrm{D}$. It is explained by the larger multipath affect to sensor 1 when near wall B at a shorter distance to sensor 2 .

- Meaning of legends in figures

- Figure 7.6

* "S1: $X^{\prime \prime}$ : Sensor 1 on Wall A is located at $X$, where $X$ is A, B, C, D, or E in Figure 7.4

- Figure 7.7 
* "S2: row $n^{\prime \prime}$ : Sensor 2 on Wall B is located at row $n$, where $n$ is one of $[1 . .7]$ in Figure 7.4

- Figure 7.8

* Subtitle "S1-WallA-X-S2-WallB": Sensor 1 on wall A is located at location $X$, where $X$ is $\mathrm{A}, \mathrm{B}, \mathrm{C}, \mathrm{D}$, or $\mathrm{E}$, and $\mathrm{S} 2$ is on wall $\mathrm{B}$ in Figure 7.4

* "S2: row $n^{\prime \prime}$ : Sensor 2 on Wall B is located at row $n$, where $n$ is one of [1..7] in Figure 7.4

The same data was plotted in Figure 7.7 per row that sensor 2 was placed. The high errors whose values were greater than $10 \%$ occurred when sensor 2 was near the room floor or ceiling (i.e. row 1, 2, 6, and 7), and the distance between sensor 1 and sensor 2 was small. As can be seen from the upper right and bottom right of subplots of Figure 7.8, when sensor 1 was near wall B the error reduced as sensor 2 moved away from sensor 1. Also, the error was high when sensor 1 was at position $\mathrm{B}$ and sensor 2 was on the row 1 or 7 near wall A or when sensor 1 was at position E and sensor 2 was on the row 1, 6 and 7 near wall $\mathrm{A}$. In other words, when the two sensors were located close to each other and near the room corners the error was high. It is explained by the severe multipath on the corner of room floor and ceiling and also by the weak received signal when the faces of the Tx and Rx antennas are offset vertically. 


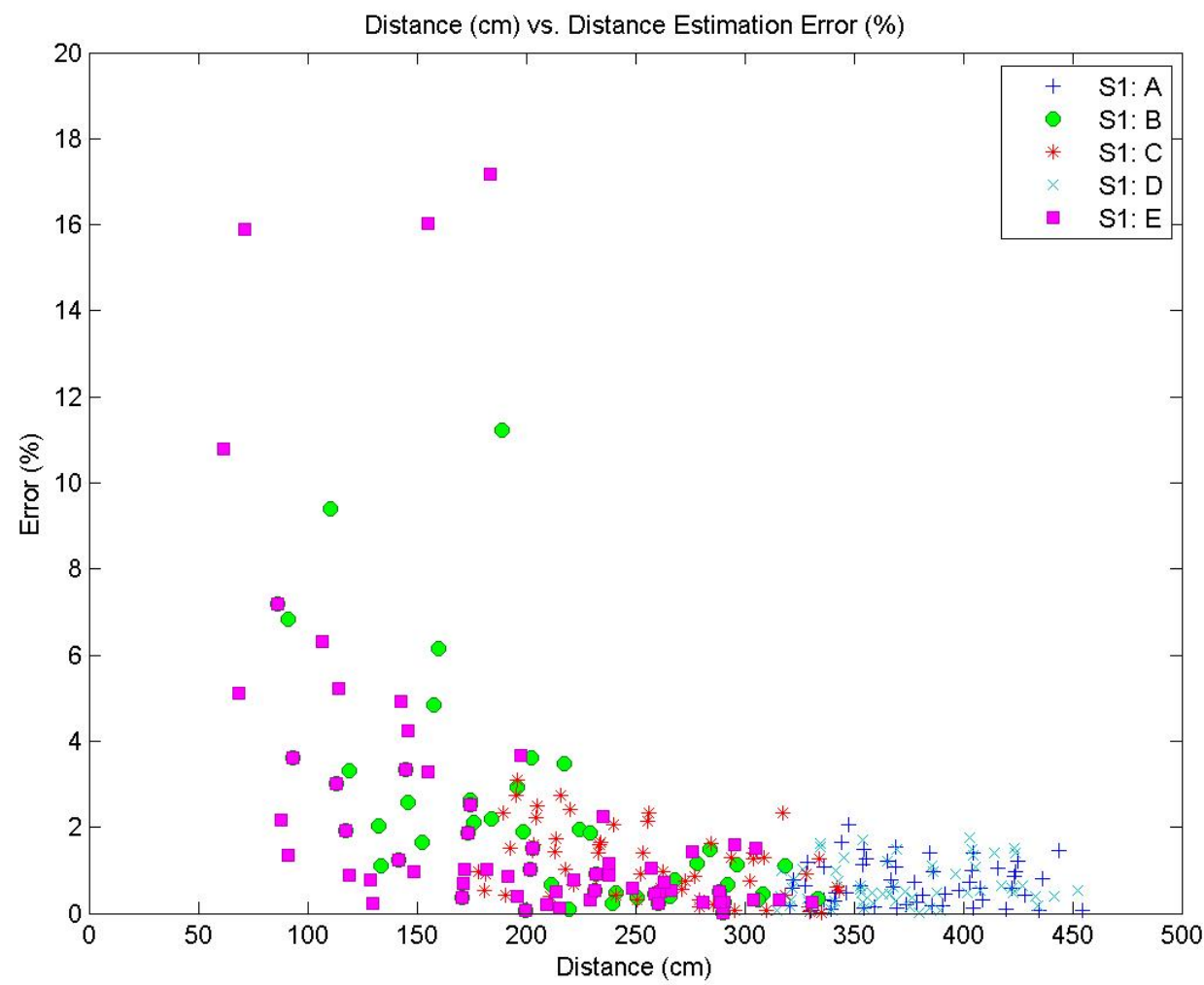

Figure 7.6. Adjoining wall distance estimation error per sensor 1 location 


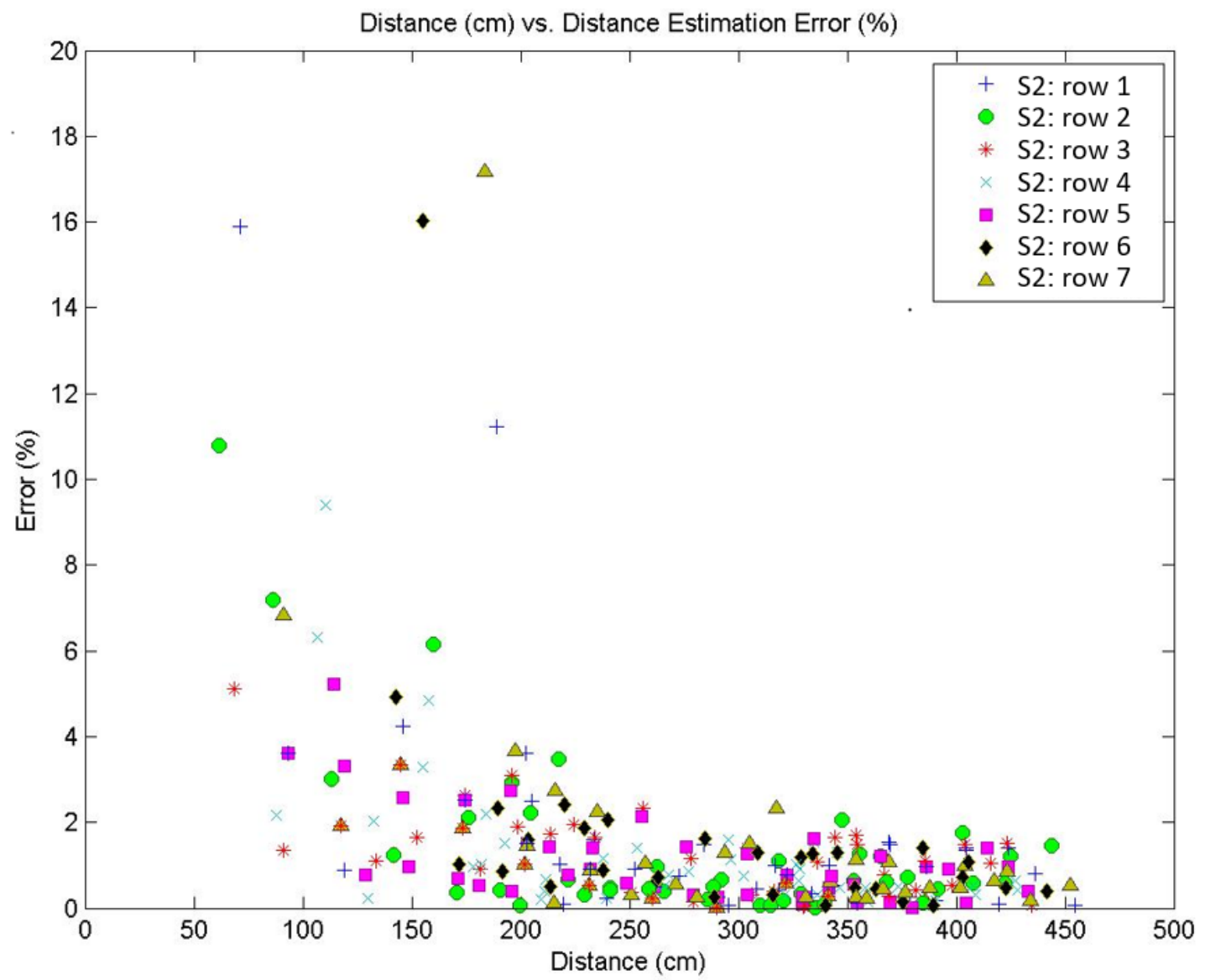

Figure 7.7. Adjoining wall distance estimation error per sensor 2 location on each row 

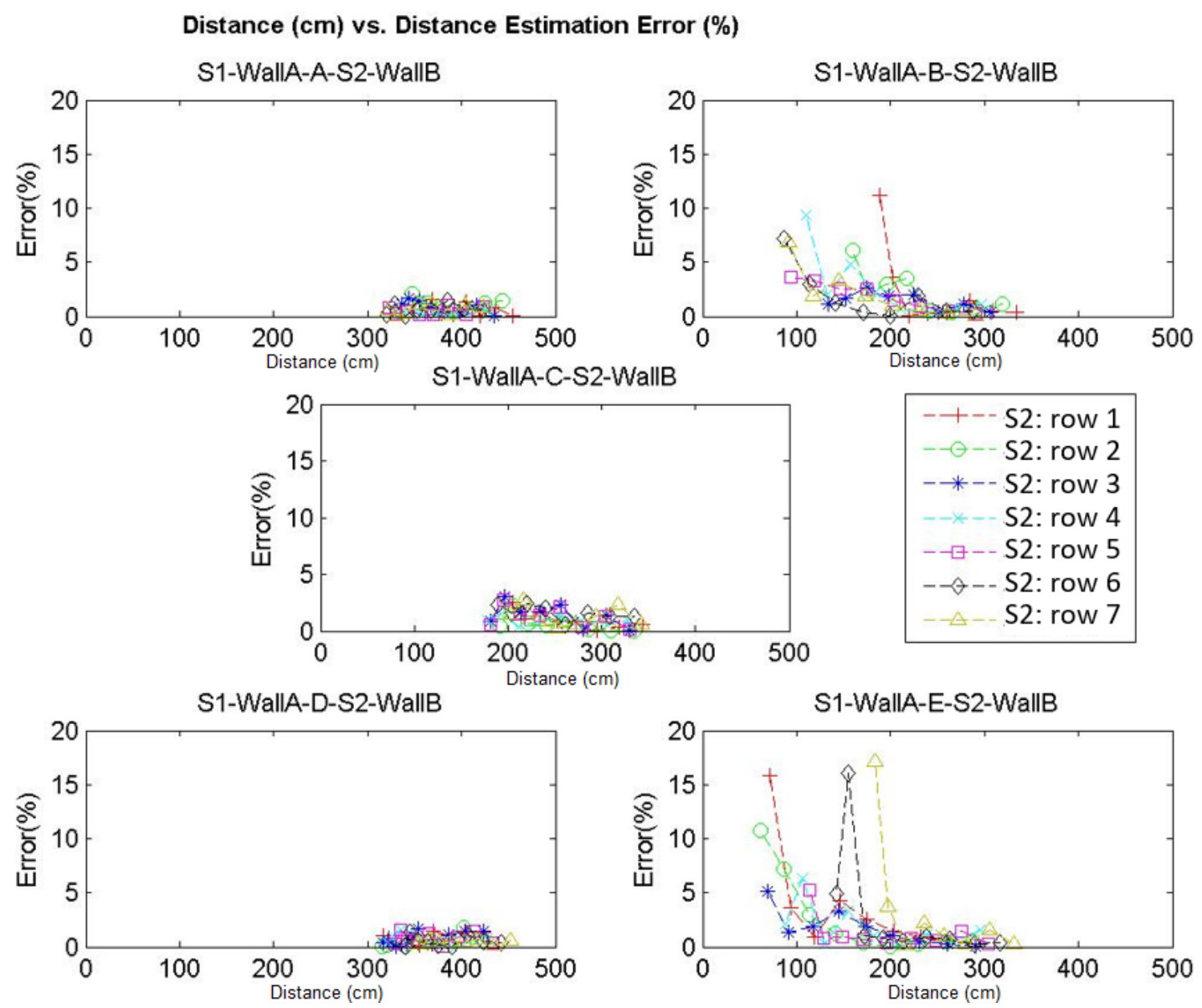

Figure 7.8. Adjoining wall distance estimation error per sensor 1 and sensor 2 location 


\subsubsection{Results for Adjoining Wall AoA Measurement}

This section discusses the results for AoA measurement when two walls are adjoining. Figure 7.9 to Figure 7.14 show the AoA estimation errors compared to actual angles. Figure 7.9 and Figure 7.10 plot AoA estimation errors from each sensor 1 position to all sensor 2 positions, in relative error and absolute error respectively. And, Figure 7.11 and Figure 7.12 plot AoA estimation errors from each sensor 2 row position to all sensor 1 positions, in relative error and absolute error respectively. For both cases, the relative error was high when the distance between the two sensors is at around 3 meters, and it became smaller as the distance between the two sensors becomes smaller or larger. This is because when the actual angle being measured is close to 0 degree, even a smaller absolute error is magnified when considering relative error. The distance between the two sensors becomes around 3 meters when sensor 1 is near wall B and sensor 2 is near wall $\mathrm{C}$, facing each other at around 0 degree. The distance between the two sensors becomes smaller when the two sensors are near the adjoining corner of wall A and wall B, and it becomes larger when sensor 1 and sensor 2 move away from the adjoining corner. In both cases, the angle the two radios form becomes large, thus the relative error becomes small. The absolute error is not large at around 3 meters as can be seen in Figure 7.10 and Figure 7.12. It is less than 10 degrees mostly.

Figure 7.13 subplots relative error on each sensor 1 position for each sensor 2 row location. It can be seen from this figure that the relative error was small when sensor 1 was in the middle of wall A or away from wall B. The relative error was high when sensor 1 was near wall B and the distance between sensor 1 and sensor 2 was large, meaning near 0 degree angle between the two sensors. It can be also seen from Figure 7.14 that the absolute error was not high in such cases. 
- Meaning of legends in figures

- Figure 7.9 and Figure 7.10

* "S1: $X^{\prime \prime}$ : Sensor 1 on Wall A is located at location $X$, where $X$ is A, B, C, D, or E in Figure 7.4

- Figure 7.11 and Figure 7.12

* "S2: row $n^{\prime \prime}$ : Sensor 2 on Wall B is located at row $n$, where $n$ is one of [1..7] in Figure 7.4

- Figure 7.13 and Figure 7.14

* Subtitle "S1-WallA-X-S2-WallB": Sensor 1 on wall A is located at location $X$, where $X$ is $\mathrm{A}, \mathrm{B}, \mathrm{C}, \mathrm{D}$, or $\mathrm{E}$, and $\mathrm{S} 2$ is on wall $\mathrm{B}$ in Figure 7.4

* "S2: row $n^{\prime \prime}$ : Sensor 2 on Wall B is located at row $n$, where $n$ is one of [1..7] in Figure 7.4 


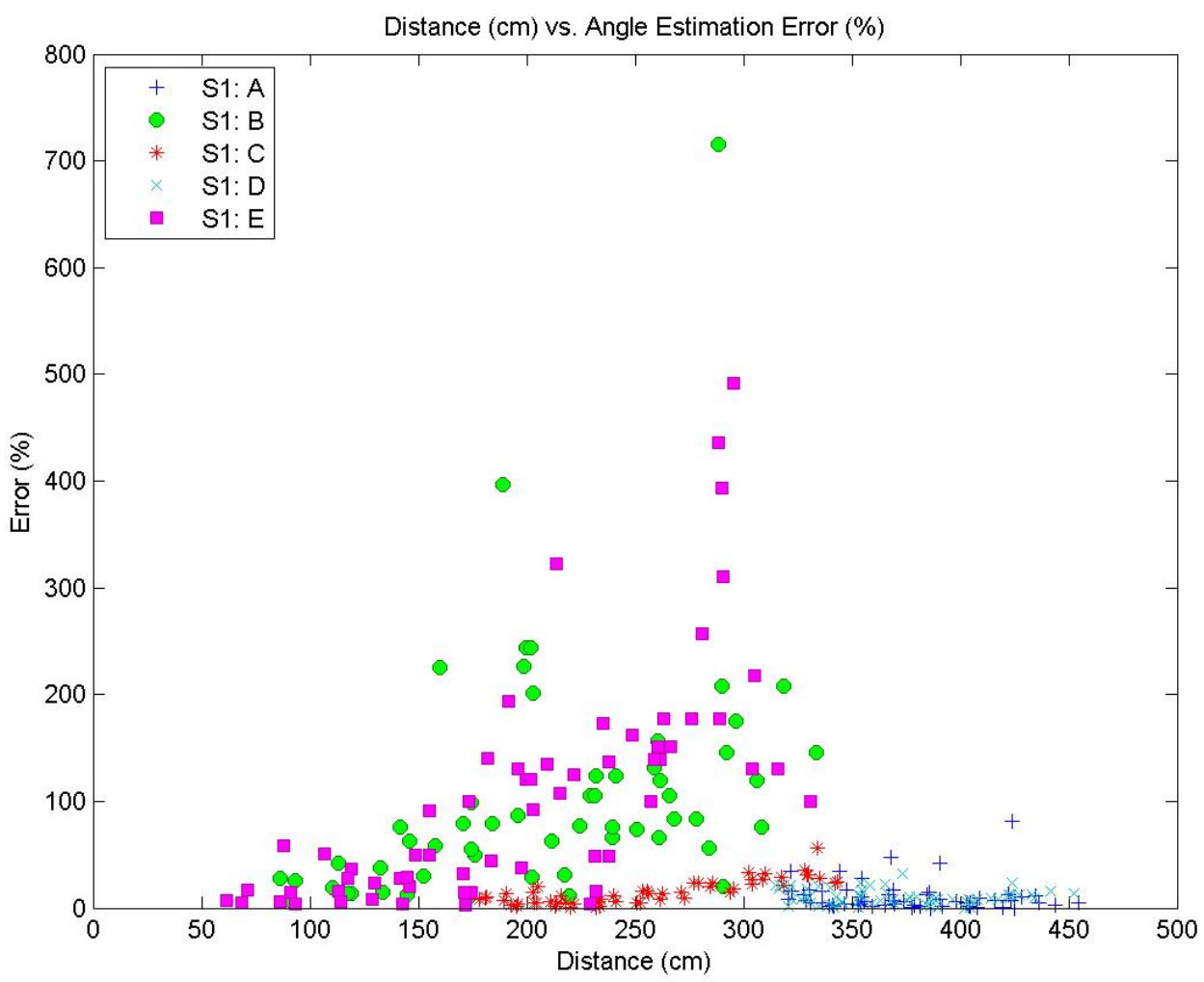

Figure 7.9. Adjoining wall angle estimation error per sensor 1 location (relative error) 


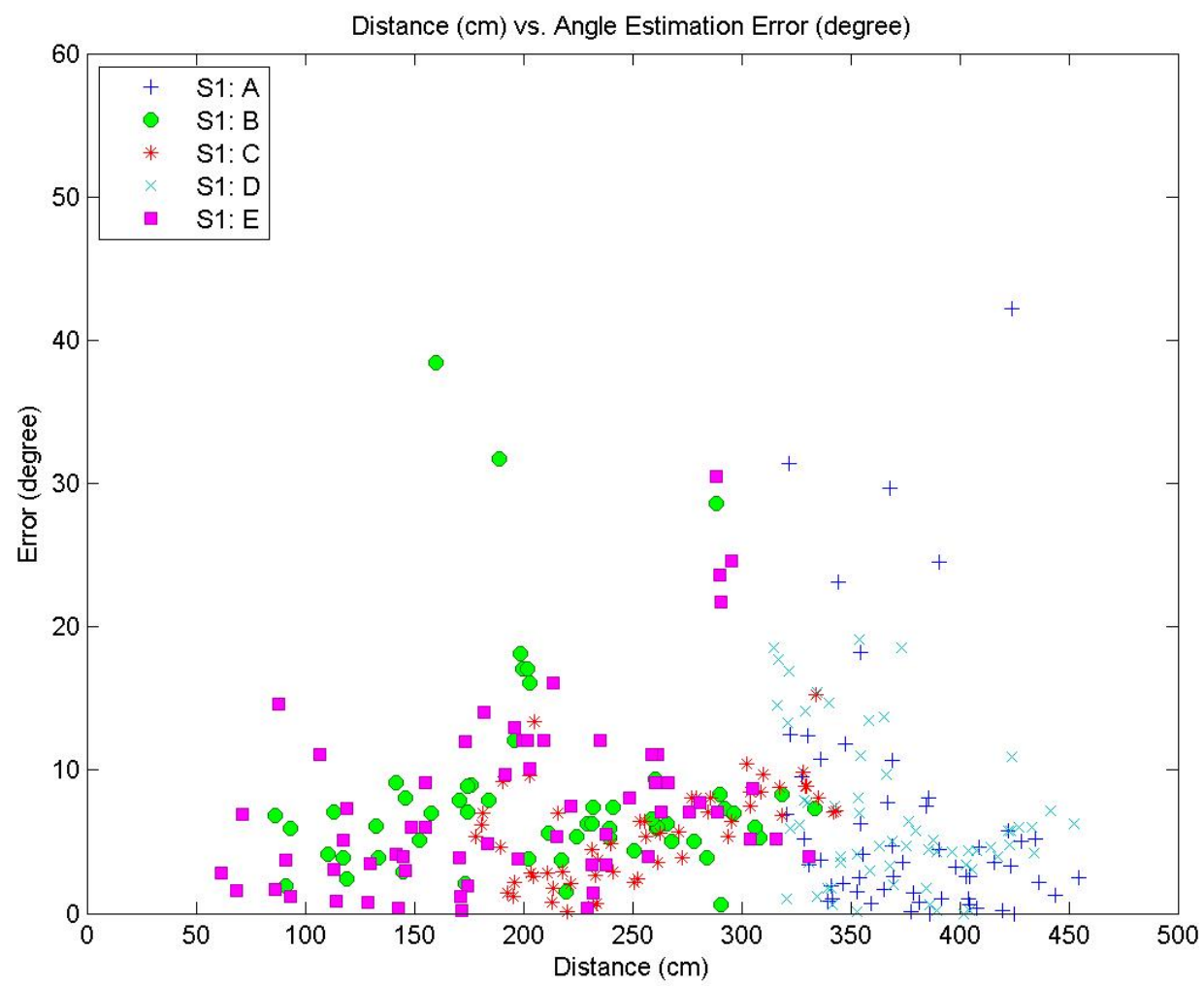

Figure 7.10. Adjoining wall angle estimation error per sensor 1 location (absolute error) 


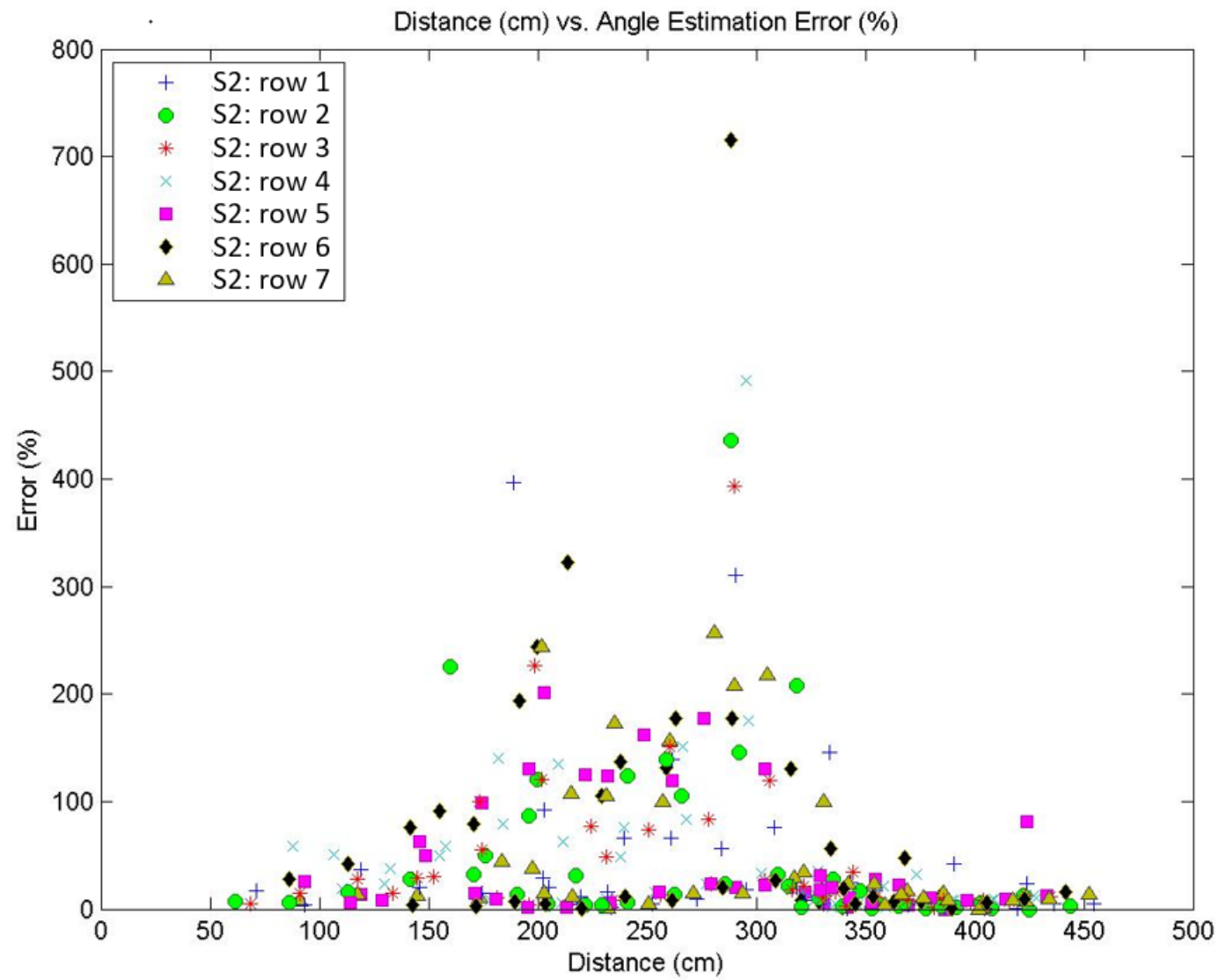

Figure 7.11. Adjoining wall angle estimation error per sensor 2 location on each row (relative error) 


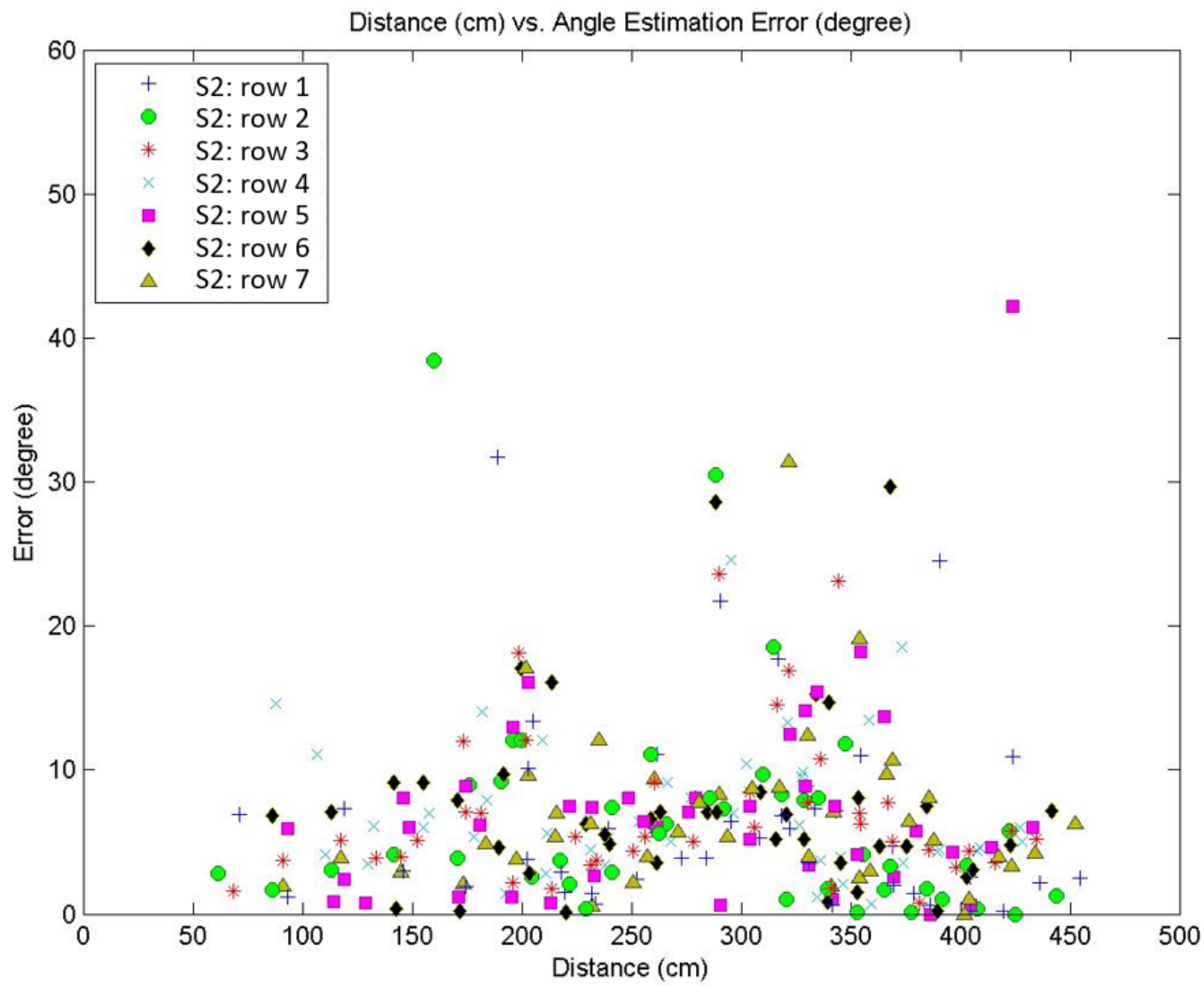

Figure 7.12. Adjoining wall angle estimation error per sensor 2 location on each row (absolute error) 

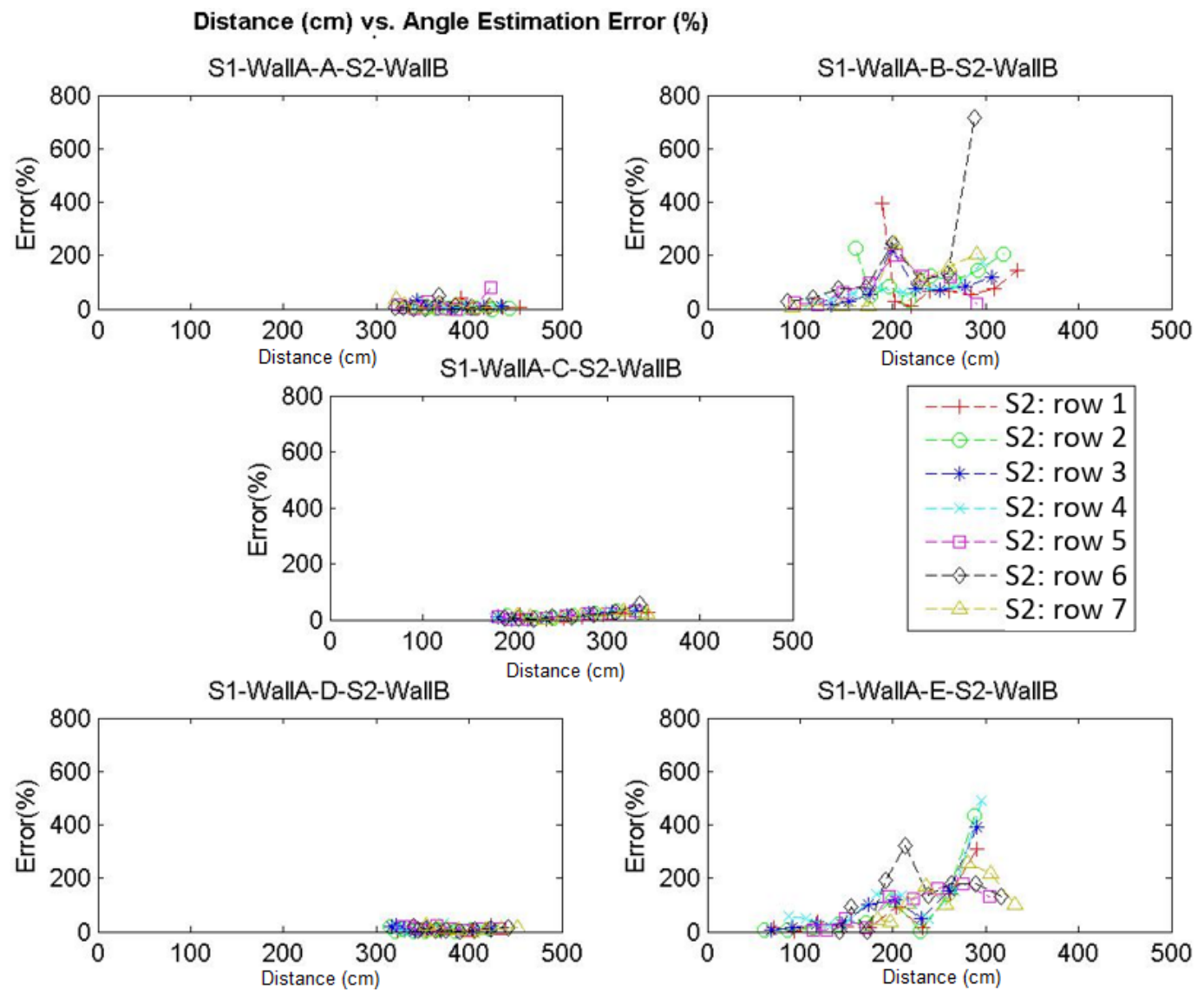

Figure 7.13. Adjoining wall angle estimation error per sensor 1 and sensor 2 location (relative error) 

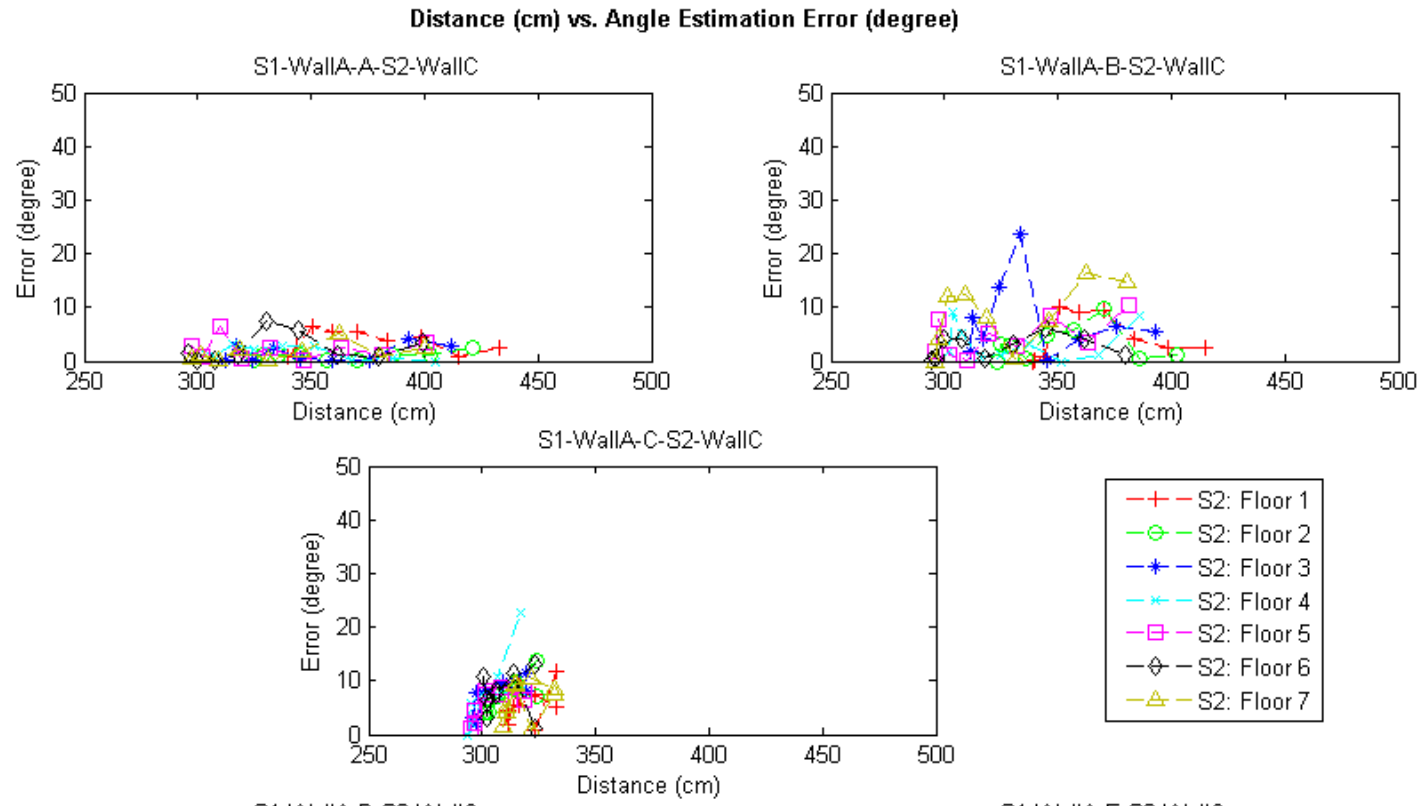

$$
\begin{aligned}
& -+-S 2 \text { : Floor } 1 \\
& -\ominus-S 2 \text { Floor } 2 \\
& -*-S 2: \text { Floor } 3 \\
& -*-S 2: \text { Floor } 4 \\
& -\boxminus-S 2: \text { Floor } 5 \\
& -\ominus-S 2: \text { Floor } 6 \\
& -A-S 2: \text { Floor } 7
\end{aligned}
$$
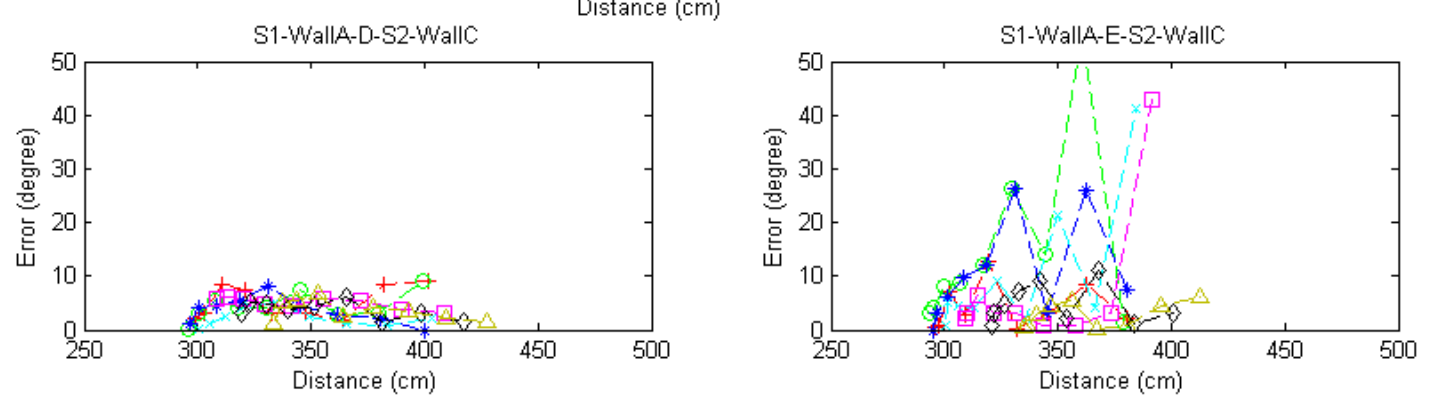

Figure 7.14. Adjoining wall angle estimation error per sensor 1 and sensor 2 location (absolute error) 


\subsubsection{Summary of Adjoining Wall Measurement Results}

This section summarizes the results for Distance and AoA measurement errors for adjoining wall. Table 7.1 summarizes the mean error and $95 \%$ error value range for Distance measurement from each S1 sensor location, e.g., position A, B, C, D and E. Mean error for distance measurement was the smallest at position D with $0.66 \%$ and the largest at position E with $2.41 \%$. As explained in section 7.3.1, due to multipath effect, the error was higher when sensor S1 was close to the adjoining wall, e.g., at position $\mathrm{B}$ and $\mathrm{E}$.

Table 7.2 summarizes the mean error and $95 \%$ error range for AoA measurement from each S1 sensor location. Mean error for AoA measurement was small at around $10 \%$ to $15 \%$ at position A, C, and D, but it was large at position B and E with about $105 \%$ error. As explained previously, the AoA error was high at position $\mathrm{B}$ and $\mathrm{E}$ since the relative angle estimation error becomes high compared to near 0 angle values.

Table 7.1. Distance measurement result (adjoining wall)

\begin{tabular}{|c|c|c|}
\hline S1 & Mean error (\%) & $\mathbf{9 5 \%}$ range (\%) \\
\hline A & 0.68 & {$[0.07,1.54]$} \\
\hline B & 2.02 & {$[0.10,7.20]$} \\
\hline C & 1.16 & {$[0.06,2.74]$} \\
\hline D & 0.66 & {$[0.06,1.62]$} \\
\hline E & 2.41 & {$[0.11,15.89]$} \\
\hline
\end{tabular}


Table 7.2. AoA measurement result (adjoining wall)

\begin{tabular}{|c|c|c|}
\hline S1 & Mean error (\%) & $\mathbf{9 5 \%}$ range (\%) \\
\hline A & 10.63 & {$[0.17,42.24]$} \\
\hline B & 105.97 & {$[11.54,244.29]$} \\
\hline C & 15.10 & {$[1.43,33.55]$} \\
\hline D & 10.31 & {$[0.34,23.19]$} \\
\hline E & 105.72 & {$[4.00,393.33]$} \\
\hline
\end{tabular}

\subsection{RESULTS FOR OPPOSITE WALL MEASUREMENT}

In this section, measurement values from distance ranging and AoA experiment for the opposite wall placement were analyzed in order to see the measurement error compared to the actual angle and actual distance.

\subsubsection{Results for Opposite Wall Distance Measurement}

This section discusses the results for distance measurement when two walls are opposite. Figure 7.15 shows the distance estimation errors compared to actual distance per sensor 1 location. Figure 7.16 is for each sensor 2 location for the same.

Most of the distance errors are within $10 \mathrm{~cm}$ error range and less than $2 \%$. The distance estimation performance of opposite wall case is observed to be better than the adjoining wall case, which had more data points with greater than $4 \%$ error. This is due to less impact of multipath and stronger line of sight signals.

The small distance measurement error can be shown in detail in Figure 7.17, where it shows subplots of distance error for each sensor 1 position, at A, B, C, 
D, and E, for all sensor 2 row locations. In all cases the error was small, i.e., less than $5 \%$, since sensor A and sensor B have enough distance space of minimum 3 meters between them.

- Meaning of legends in figures

- Figure 7.15

* "S1: $X^{\prime \prime}$ : Sensor 1 on Wall A is located at location $X$, where $X$ is A, B, C, D, or E in Figure 7.5

- Figure 7.16

* "S2: row $n^{\prime \prime}$ : Sensor 2 on Wall $\mathrm{C}$ is located at row $n$, where $n$ is one of [1..7] in Figure 7.5

- Figure 7.17

* Subtitle "S1-WallA-X-S2-WallC": Sensor 1 on wall A is located at location $X$, where $X$ is $\mathrm{A}, \mathrm{B}, \mathrm{C}, \mathrm{D}$, or $\mathrm{E}$, and $\mathrm{S} 2$ is on wall $\mathrm{C}$ in Figure 7.5

* "S2: row $n^{\prime \prime}$ : Sensor 2 on Wall $\mathrm{C}$ is located at row $n$, where $n$ is one of [1..7] in Figure 7.5

\subsubsection{Results for Opposite Wall AoA Measurement}

This section shows the AoA estimation errors compared to actual angles when sensor 1 and sensor 2 were placed on the opposite walls. Figure 7.18 plots relative AoA estimation errors from each sensor 1 position to all sensor 2 positions. And Figure 7.19 plots relative AoA estimation errors from each sensor 2 row position to all sensor 1 positions. For both cases, the relative error was high when the distance between the two sensors is small at around 3 meters, and it became smaller as the 


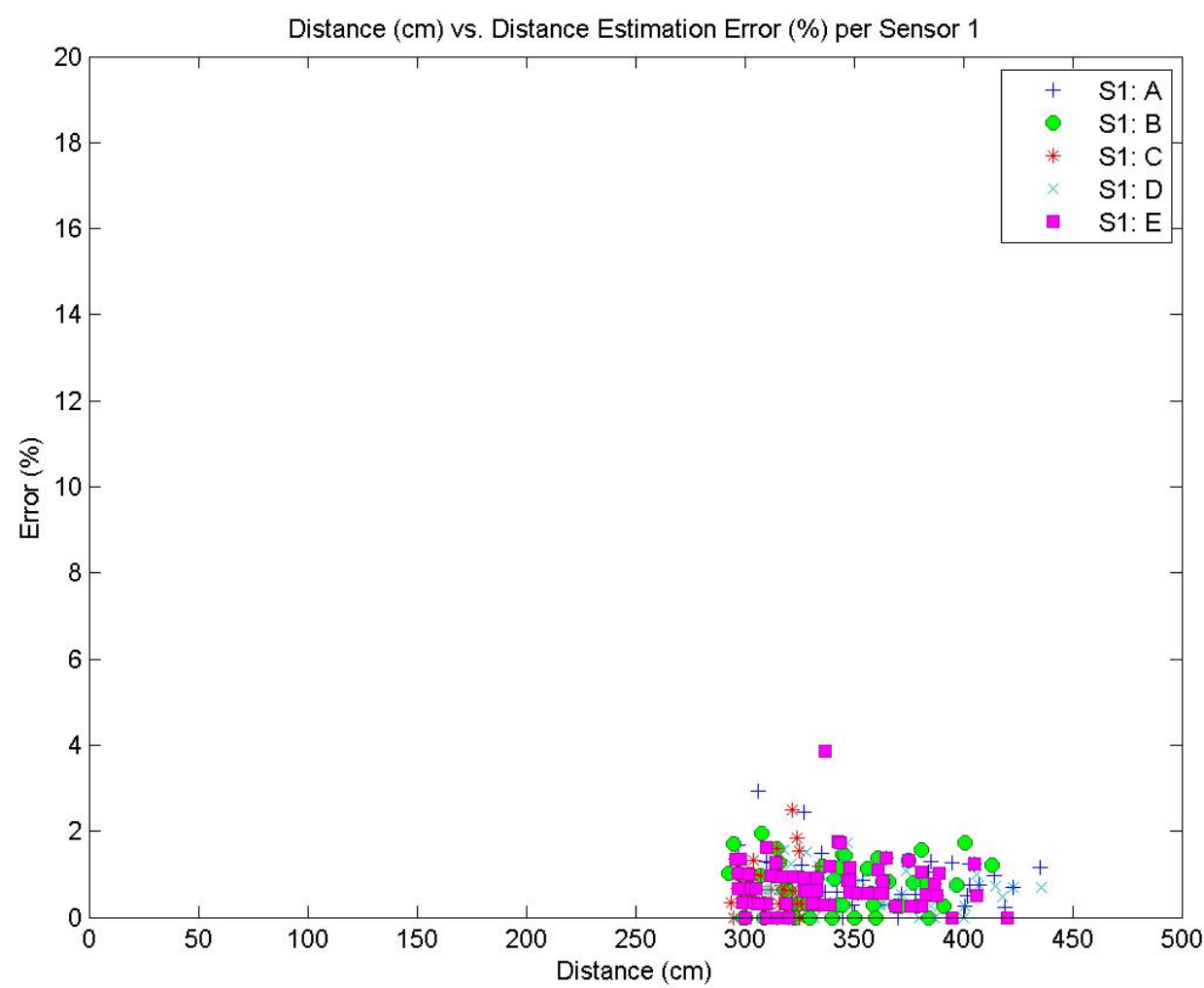

Figure 7.15. Opposite wall distance estimation error per sensor 1 location 


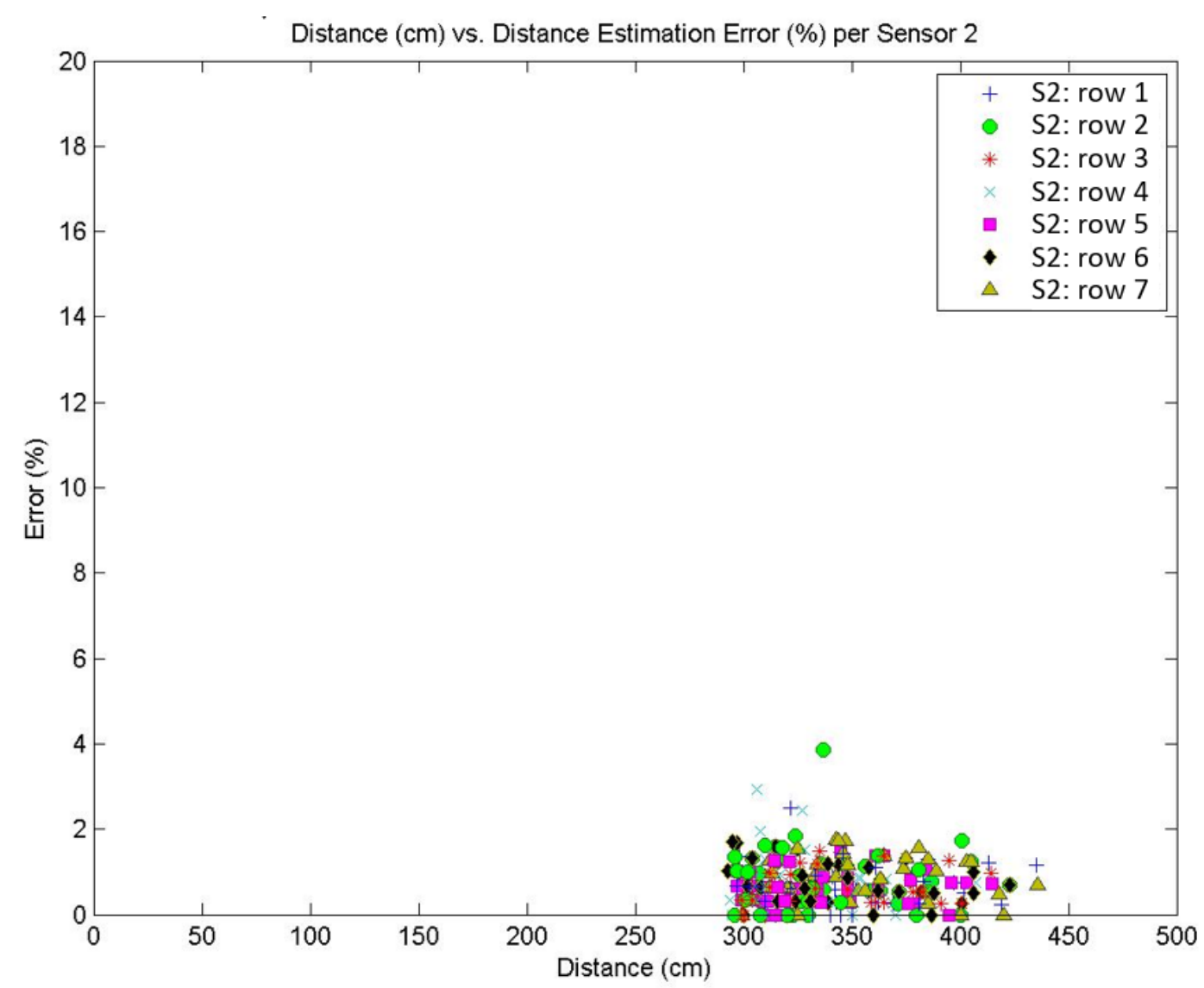

Figure 7.16. Opposite wall distance estimation error per sensor 2 location on each row 

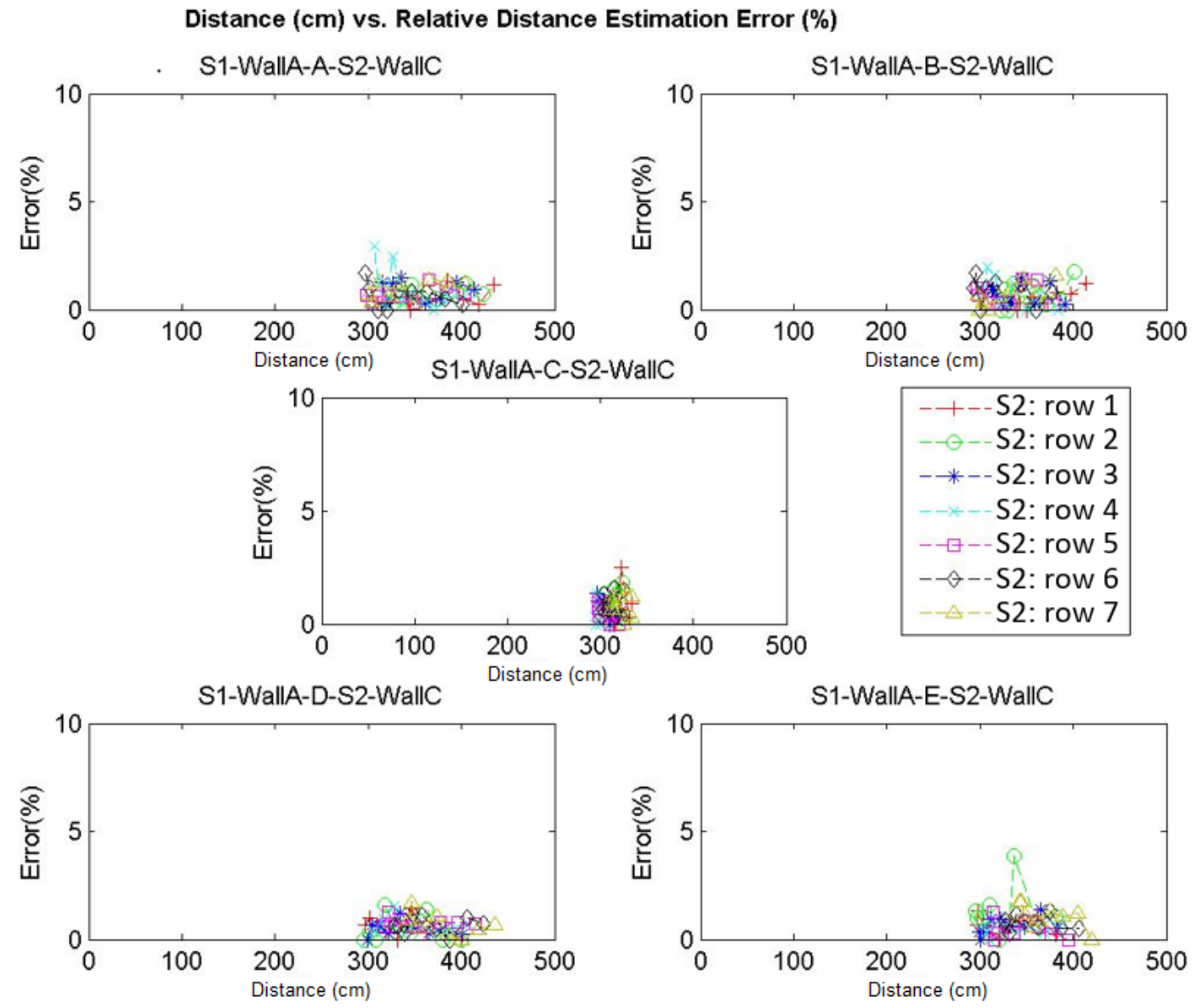

Figure 7.17. Opposite wall distance estimation error per sensor 1 and sensor 2 location 
distance between the two becomes larger. This is because when the actual angle being measured is close to 0 , even a small absolute error is magnified when we consider relative error. The distance between the two sensors is about 3 meters when the two are facing each other at around 0 degree. Absolute error is not big at around 3 meters as can be seen in Figure 7.20, but the relative error compared to 0 degree gives large relative error as shown in Figure 7.21. Overall most of the angle measurement error was less than $50 \%$ when the two sensors were at oblique angles. It is worthwhile to note that, due to the ceiling fan installed in the experimental room, there was higher error for certain positions for the reference node and the target node when close to the top.

- Meaning of legends in figures

- Figure 7.18

* "S1: $X^{\prime \prime}$ : Sensor 1 on Wall A is located at location $X$, where $X$ is $\mathrm{A}, \mathrm{B}, \mathrm{C}, \mathrm{D}$, or E in Figure 7.5

- Figure 7.19

* "S2: row $n^{\prime \prime}$ : Sensor 2 on Wall $\mathrm{C}$ is located at row $n$, where $n$ is one of [1..7] in Figure 7.5

- Figure 7.20 \& Figure 7.21

* Subtitle "S1-WallA-X-S2-WallC": Sensor 1 on wall A is located at location $X$, where $X$ is $\mathrm{A}, \mathrm{B}, \mathrm{C}, \mathrm{D}$, or $\mathrm{E}$, and $\mathrm{S} 2$ is on wall $\mathrm{C}$ in Figure 7.5

* "S2: row $n^{\prime \prime}$ : Sensor 2 on Wall $\mathrm{C}$ is located at row $n$, where $n$ is one of [1..7] in Figure 7.5 


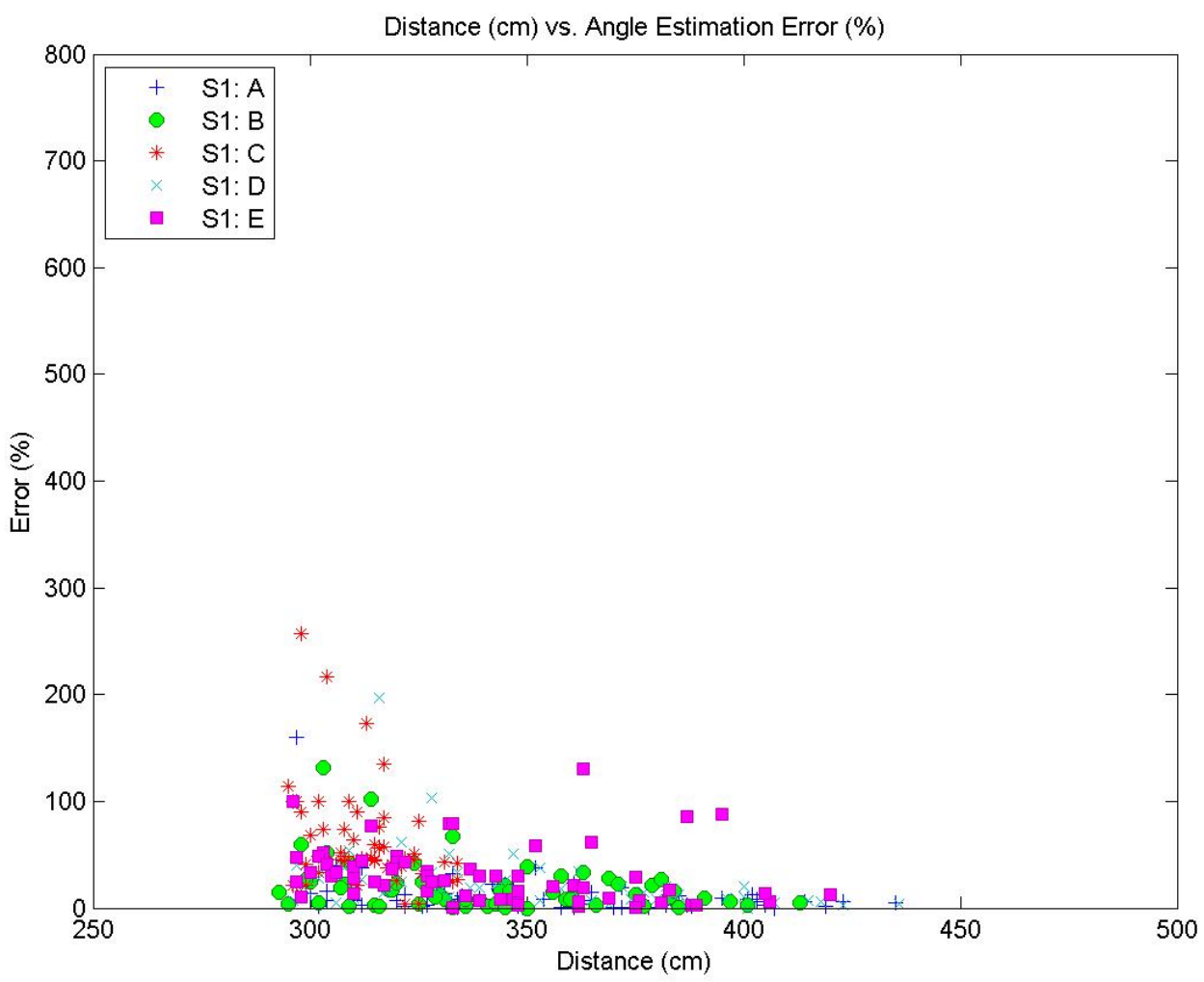

Figure 7.18. Opposite wall angle estimation error per sensor 1 location 


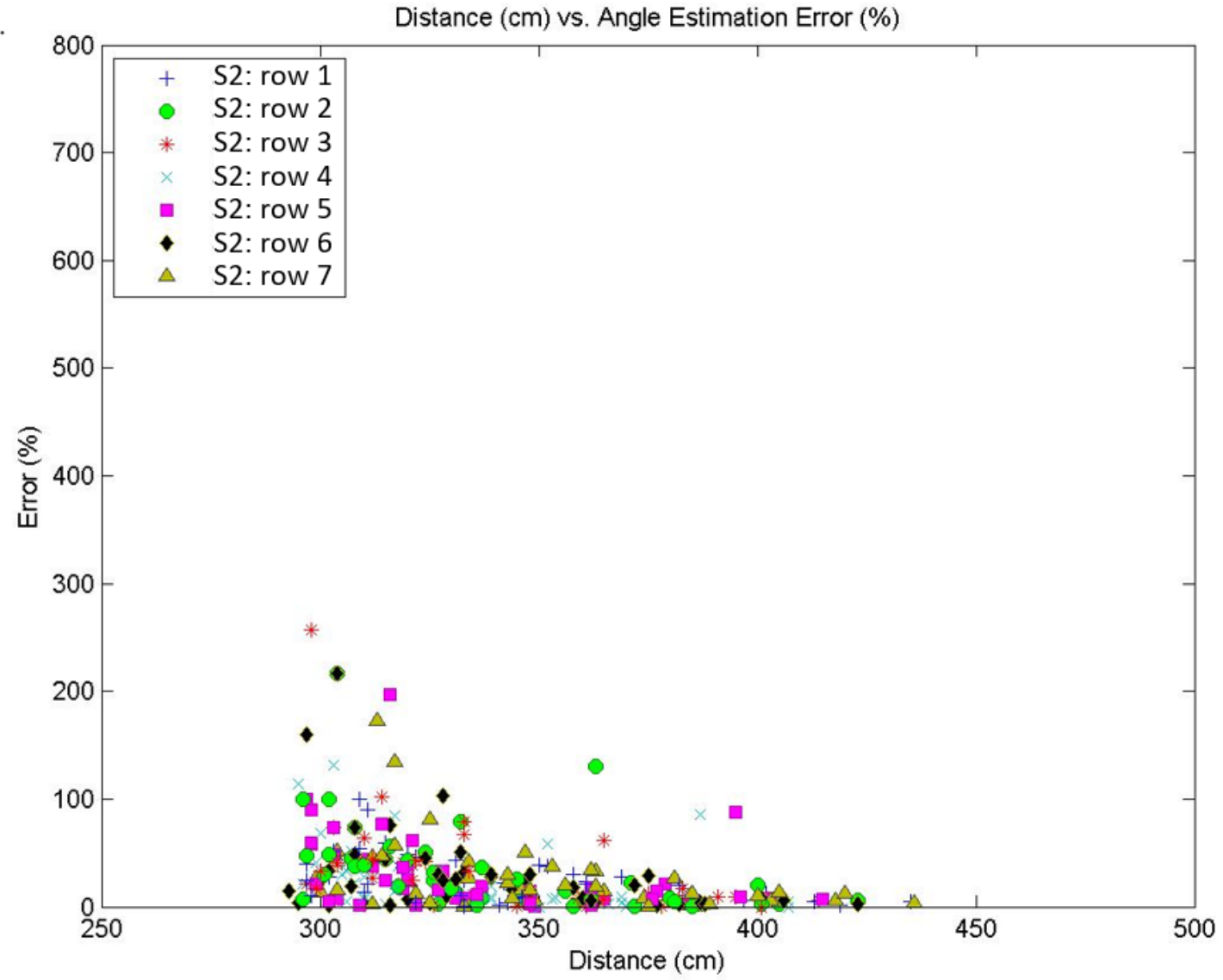

Figure 7.19. Opposite wall angle estimation error per sensor 2 on each row 

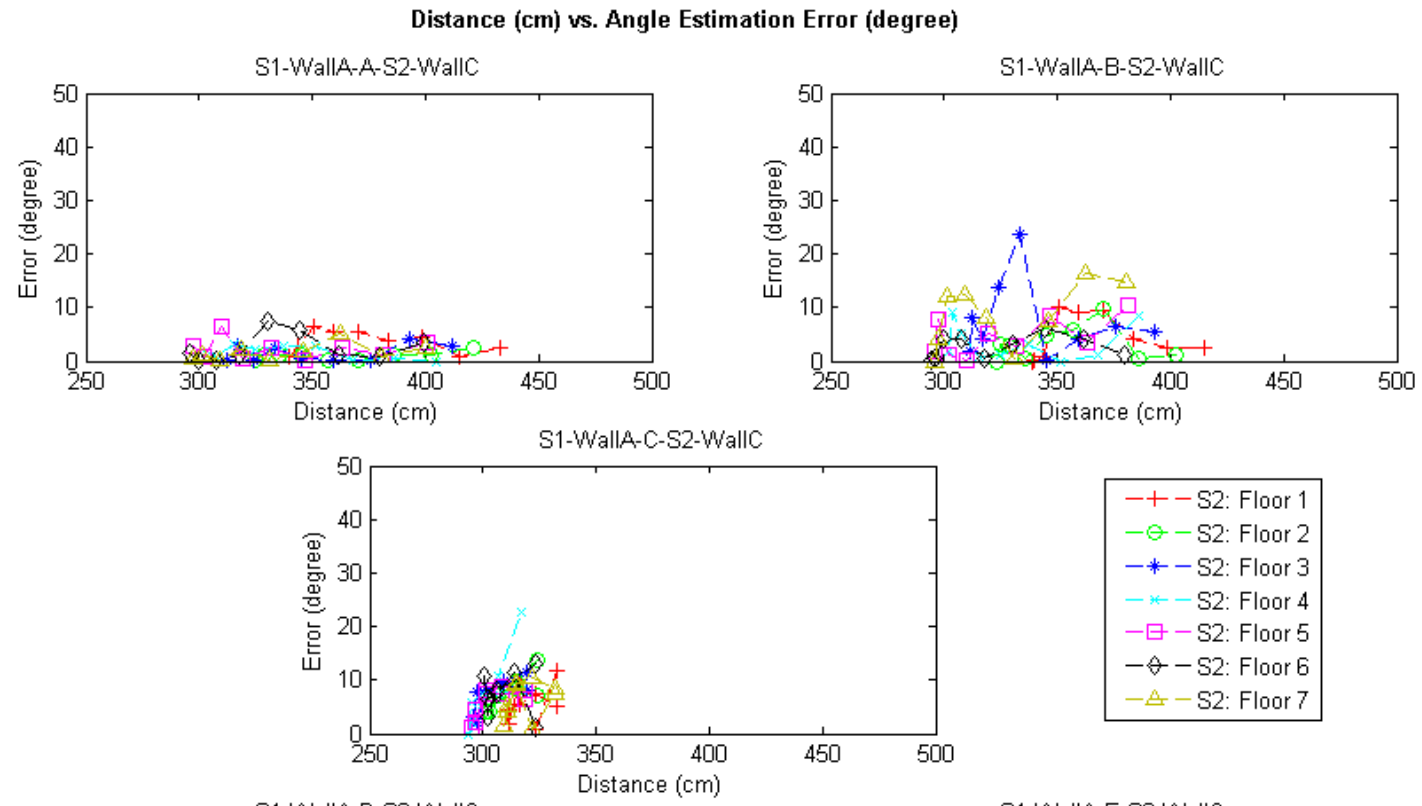

$$
\begin{aligned}
& -+-S 2 \text { : Floor } 1 \\
& -\ominus-S 2 \text { Floor } 2 \\
& -*-S 2 \text { Floor } 3 \\
& -*-S 2 \text { Floor } 4 \\
& -\boxminus-S 2 \text { Floor } 5 \\
& -\ominus-S 2 \text { Floor } 6 \\
& -A-S 2: \text { Floor } 7
\end{aligned}
$$
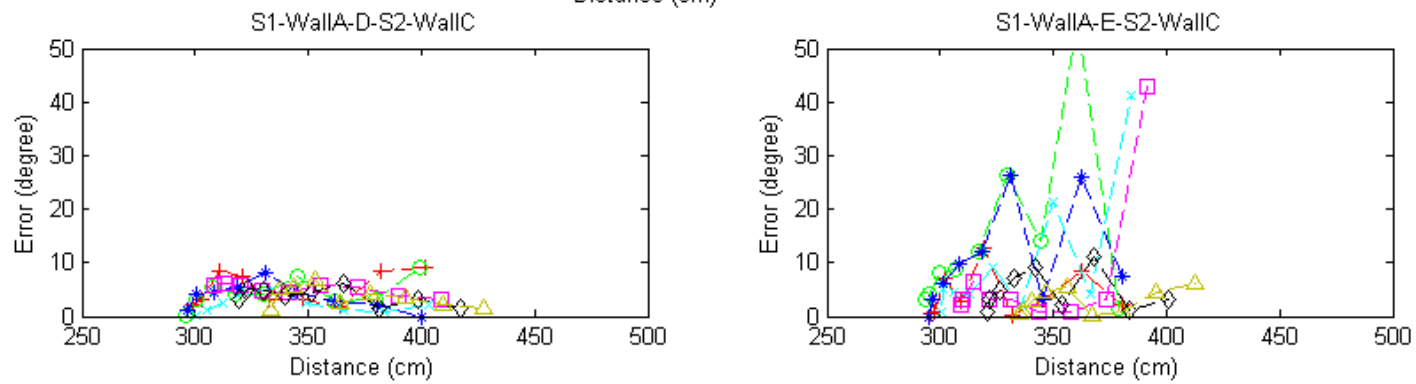

Figure 7.20. Opposite wall angle estimation error per sensor 1 and sensor 2 location (absolute error) 


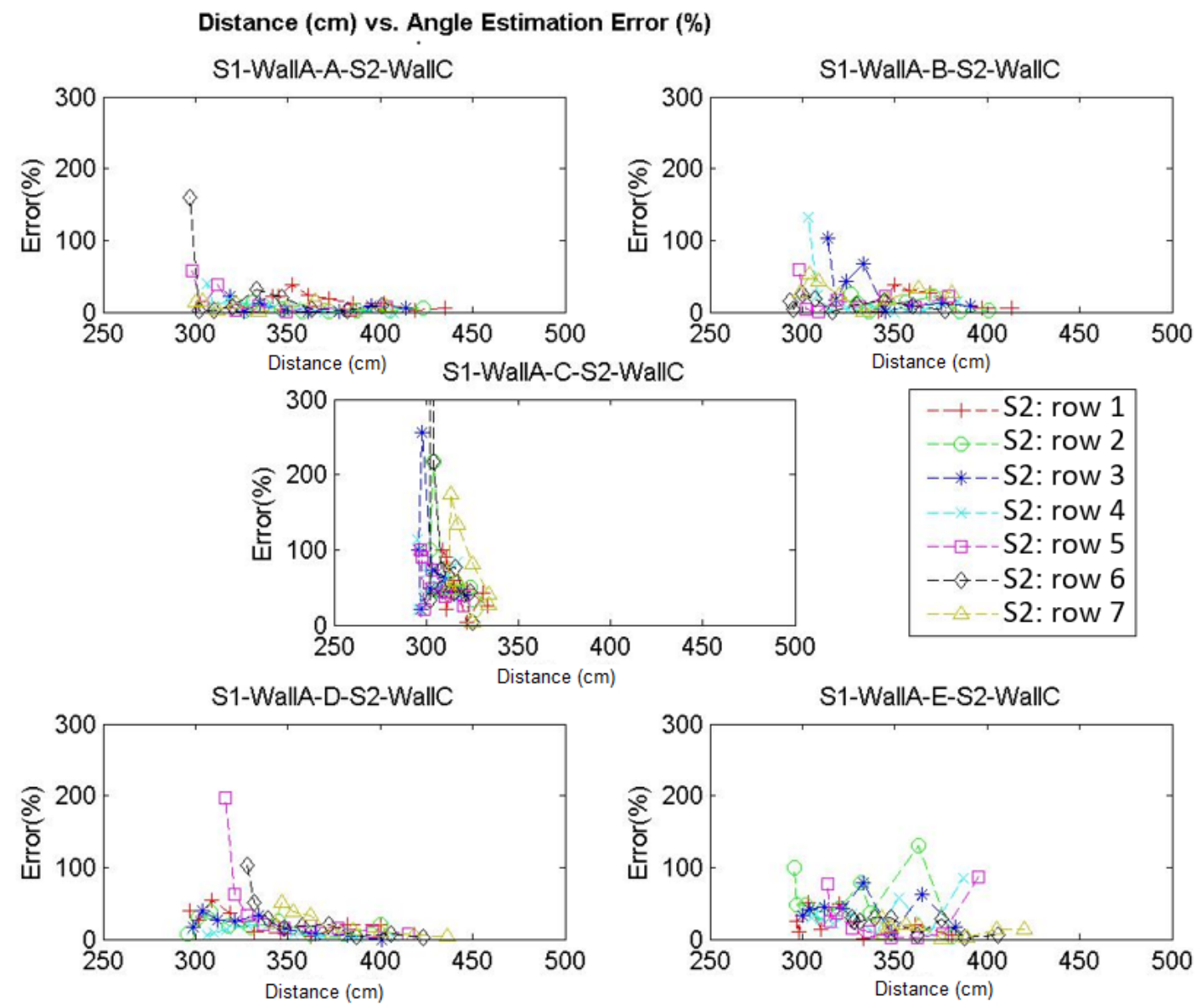

Figure 7.21. Opposite wall angle estimation error per sensor 1 and sensor 2 location (relative error) 


\subsubsection{Summary of Opposite Wall Measurement Results}

This section summarizes the results for Distance and AoA measurement errors for opposite wall. Table 7.3 summarizes the mean error and $95 \%$ error value range for Distance measurement from each S1 sensor location, e.g., position A, B, C, D and E. Distance mean error was less than $1 \%$ and $95 \%$ of errors were less than $2 \%$ in all cases. The error was smaller compared to adjoining wall case because sensor 1 and sensor 2 had good distance separation between them in all settings.

Table 7.4 summarizes the mean error and $95 \%$ error range for AoA measurement from each S1 sensor location. Mean error for AoA measurement was larger at position $\mathrm{C}$ with $80 \%$ mean error due to the same reason as the relative angle estimation error becoming high compared to near 0 angle values.

Table 7.3. Distance measurement result (opposite wall)

\begin{tabular}{|c|c|c|}
\hline S1 & Mean error (\%) & $\mathbf{9 5 \%}$ range (\%) \\
\hline A & 0.79 & {$[0.00,1.68]$} \\
\hline B & 0.72 & {$[0.00,1.69]$} \\
\hline C & 0.72 & {$[0.00,1.59]$} \\
\hline D & 0.61 & {$[0.00,1.52]$} \\
\hline E & 0.76 & {$[0.00,1.74]$} \\
\hline
\end{tabular}


Table 7.4. AoA measurement result (opposite wall)

\begin{tabular}{|c|c|c|}
\hline S1 & Mean error (\%) & 95\% range (\%) \\
\hline A & 12.75 & {$[0.29,40.00]$} \\
\hline B & 19.76 & {$[1.00,67.43]$} \\
\hline C & 80.25 & {$[4.14,216.67]$} \\
\hline D & 23.18 & {$[3.11,62.00]$} \\
\hline E & 30.97 & {$[1.75,87.35]$} \\
\hline
\end{tabular}

\subsection{RESULTS FOR ADJOINING WALL ESTIMATION}

This section explains the result of the target node (e.g. sensor 2) location estimation from the reference nodes (e.g. multiple sensor 1's) on the adjoining wall placement.

The position of the target node is calculated based on distance measurement from the reference nodes following the algorithm explained in section 7.1. Estimated position coordinate of the target node can be a complex number, for example, when a set of reference nodes form a straight line, e.g. $S 1(A, C, E)$ or $S 1(B, C, D)$ that corresponds to cyan and yellow line in Figure 7.22 respectively. $S 1(X, Y, Z)$ denotes that a set of reference nodes, $X, Y$, and $Z$, are used in order to estimate the target node location. Also, it happens when the two antennas from the reference node and the target node on the same elevation are not facing each other but edgeon or the elevation difference is high. Complex number results were eliminated for analysis.

Figure 7.22 shows all estimated target node positions by connecting them with a line from the actual target node position. Figure 7.23 excludes estimations with 


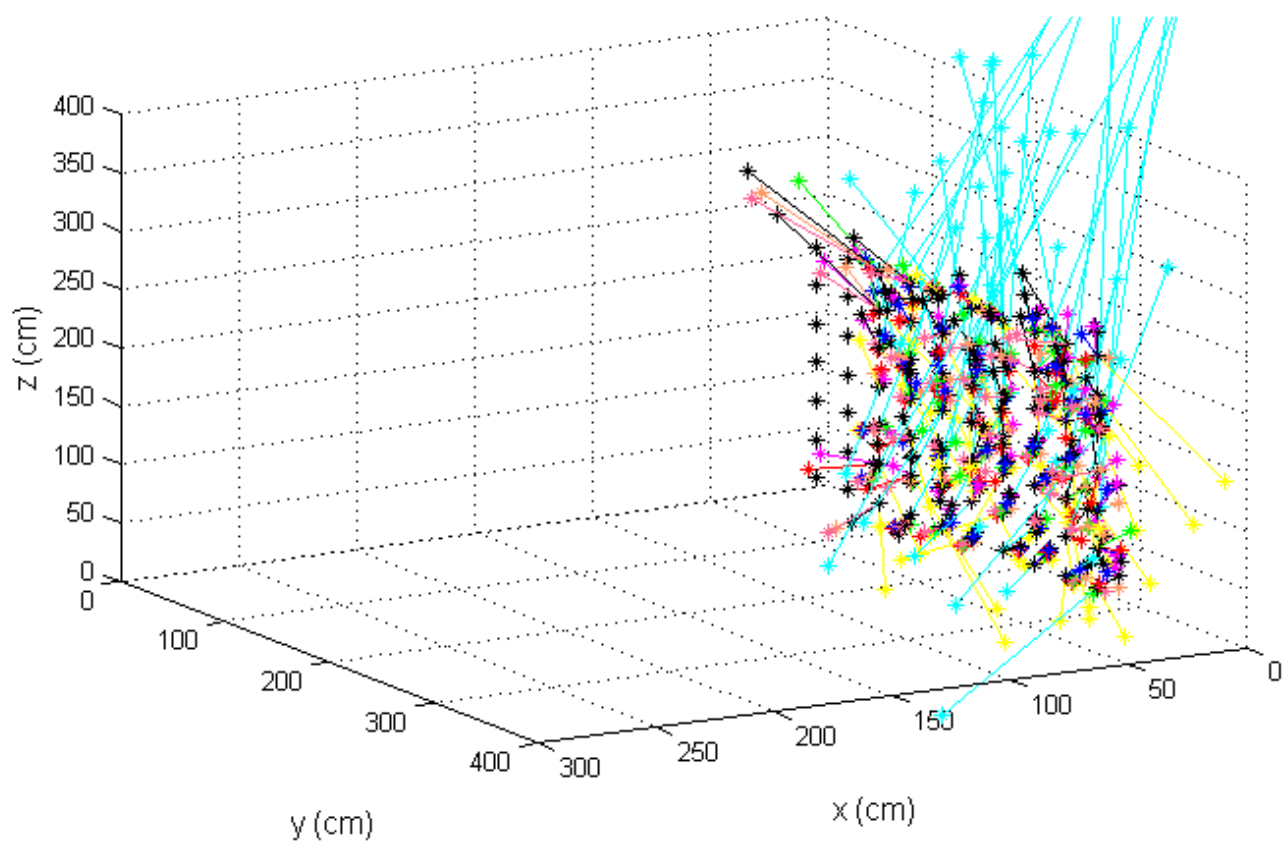

Figure 7.22. Estimated target positions with connected lines from actual target positions (Adjoining wall)

imaginary numbers from Figure 7.22. Figure 7.24 shows final result that further excludes estimations from a set of reference nodes that form a straight line, i.e. $S 1(A, C, E)$ and $S 1(B, C, D)$.

From the final set of result in Figure 7.24, the target position estimation error was calculated by calculating Euclidean distance between the actual target node position and the estimated position. Next, the mean square error (MSE) of target position estimation error was calculated for each target node. Figure 7.25 shows the cumulative probability plot of the MSE distribution of target position estimation error. It can be seen that $90 \%$ of estimation error is less than $25 \mathrm{~cm}$. The mean MSE of target position estimation error was $16.73 \mathrm{~cm}$ (Table 7.5). 


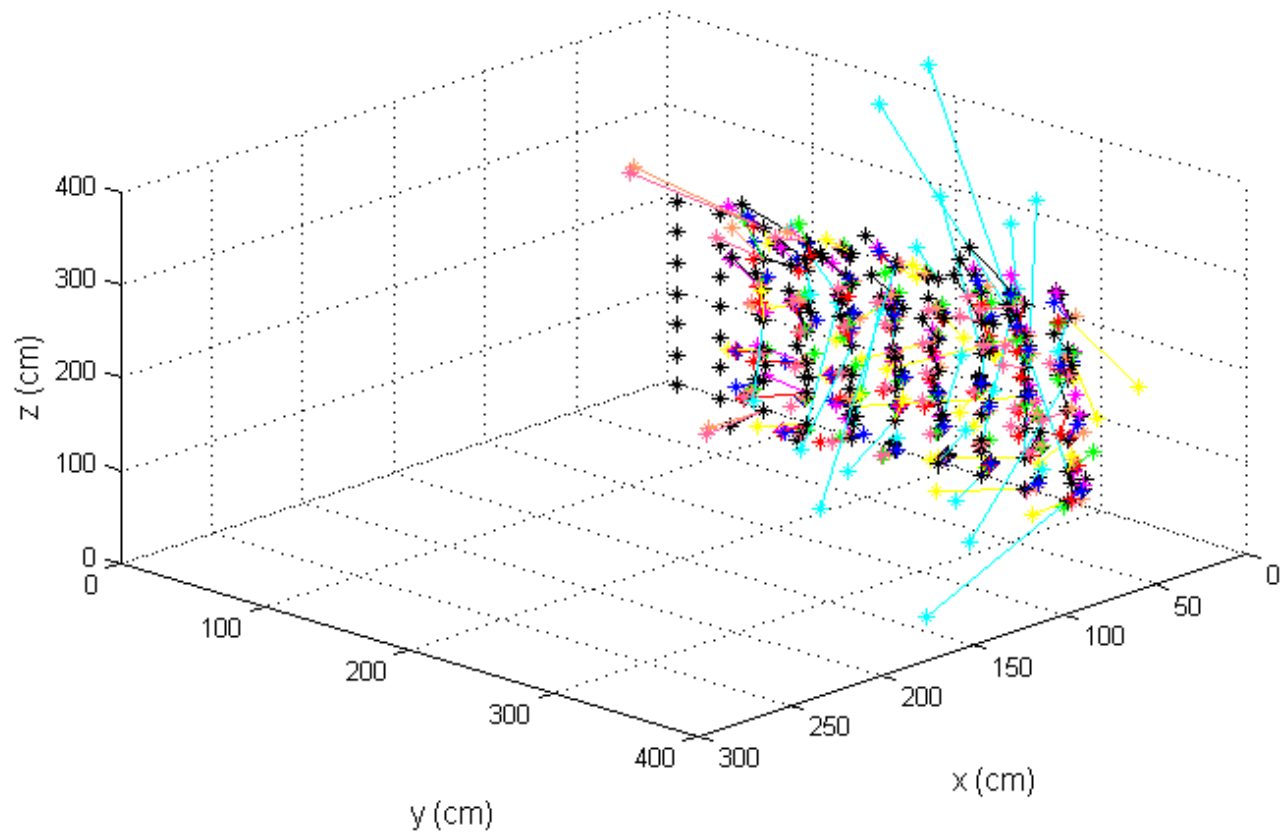

Figure 7.23. Estimated target positions that exclude estimations with imaginary numbers (Adjoining wall) 


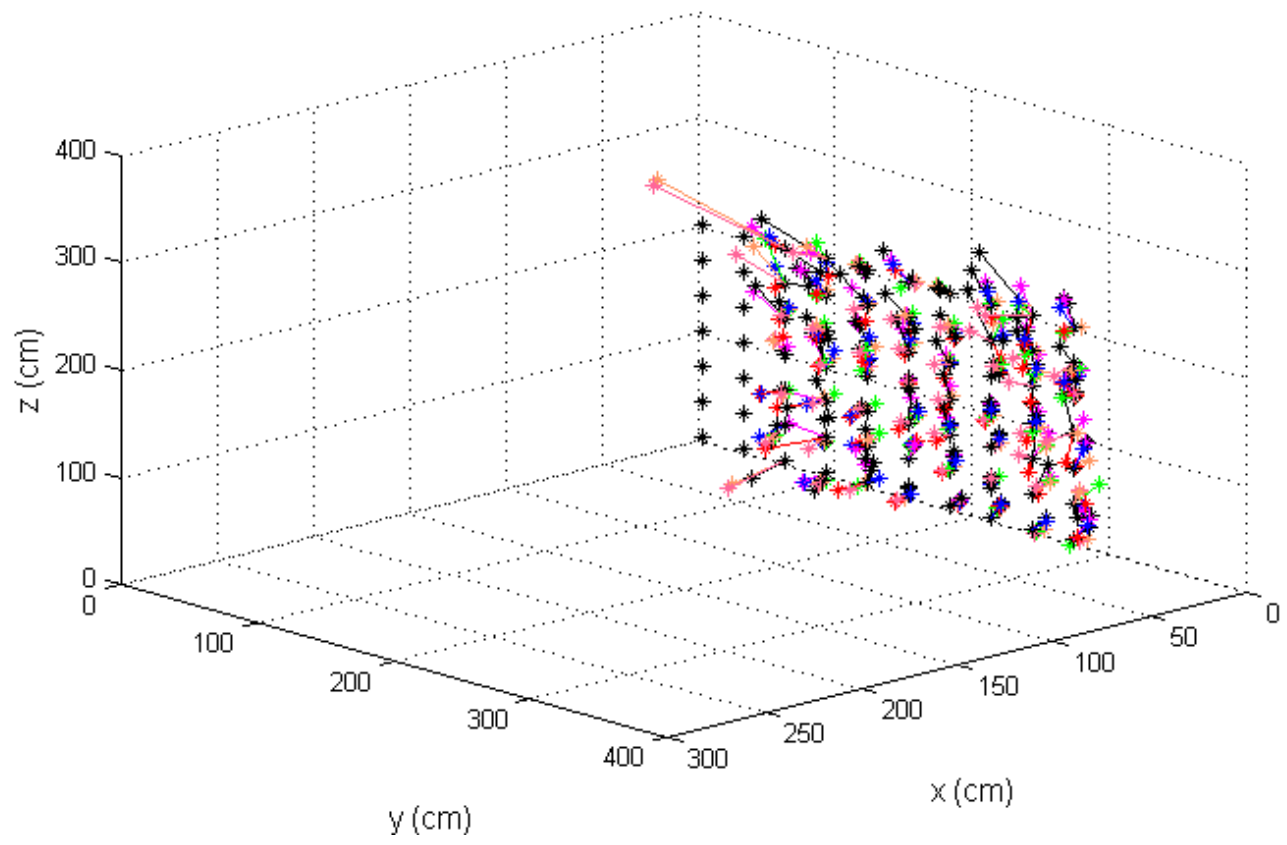

Figure 7.24. Estimated target positions with excluded estimations with imaginary numbers and from straight line reference nodes (Adjoining wall) 


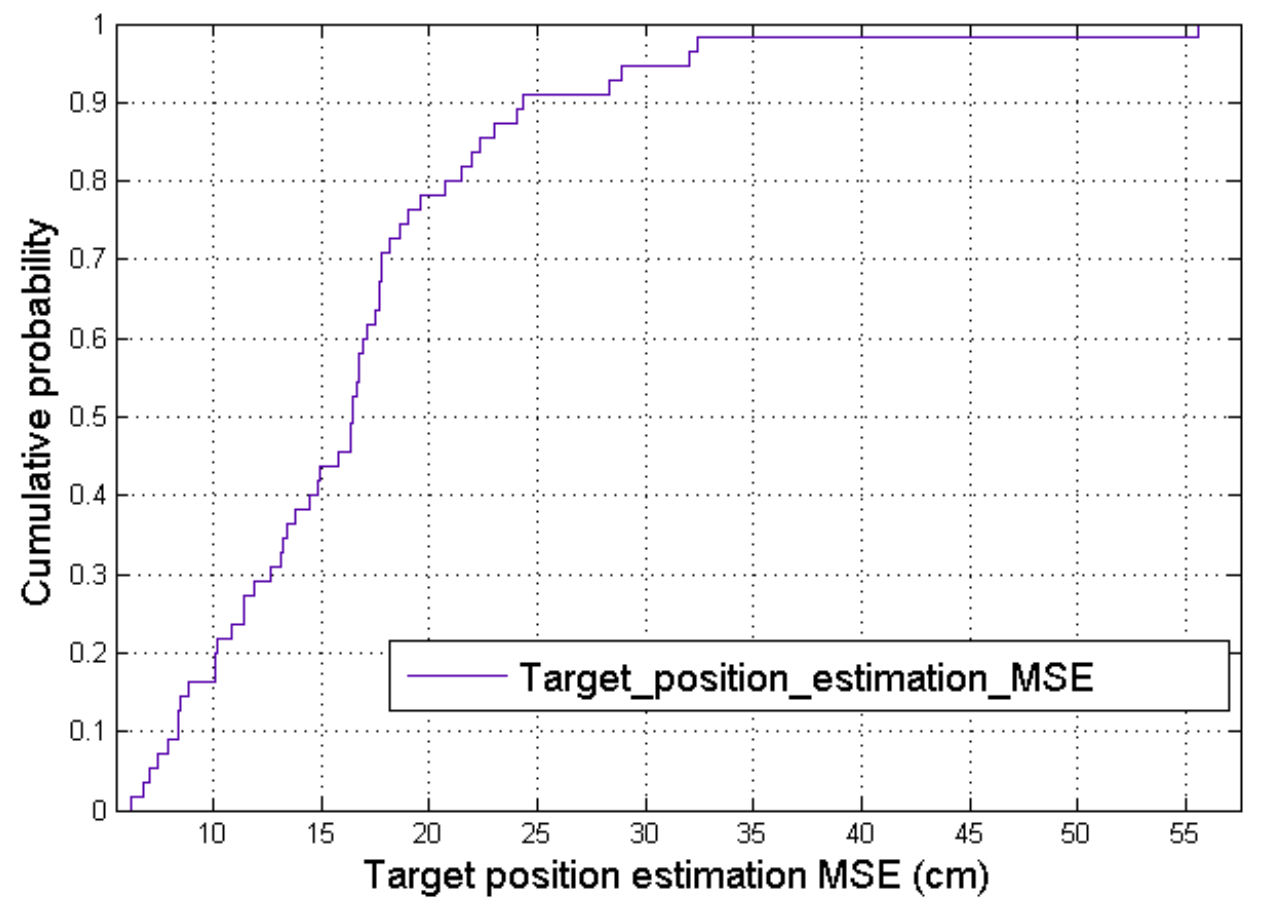

Figure 7.25. CDF of target node position estimation MSE (adjoining wall) 
Table 7.5. Target node estimation result (adjoining wall)

\begin{tabular}{|l|l|}
\hline Description (Adjoining Wall) & Mean of MSE \\
\hline Location estimation error between actual tar- & $16.73(\mathrm{~cm})$ \\
get position and estimated positions & \\
\hline
\end{tabular}

The combination of three reference nodes out of five (i.e. A to E) that gives minimum error for each target node is shown in Figure 7.26. It shows normalized error as a percentage of the inter-target node distance of $30 \mathrm{~cm}$. Most of errors were less than $30 \%$. It means that the best guesses had error of less than $9 \mathrm{~cm}$. Mean of all minimum position estimation errors was $6.25 \mathrm{~cm}$. We summarize all observations from Figure 7.26 below:

- Combinations of $(A, B, E),(B, C, E)$ and $(B, D, E)$ gives more accurate results than others, which all had position B and E. B and E are close to the adjoining wall and thus antennas of reference nodes form bigger angle with target nodes and it covers wider elevations from floor to ceiling.

- Combinations such as $(A, C, E)$ and $(B, C, D)$ where reference nodes lie on a straight line don't perform well.

- $(A, C, D)$ where all reference nodes are far from the adjoining wall also does not produce good estimation because antennas form smaller angle with target node.

- $N a N$ indicates that target nodes at 30 and $60 \mathrm{~cm}$ mark for each row failed to get estimation from any of the combinations because they are either too close to reference nodes or antenna angles are at near 0 degrees. 


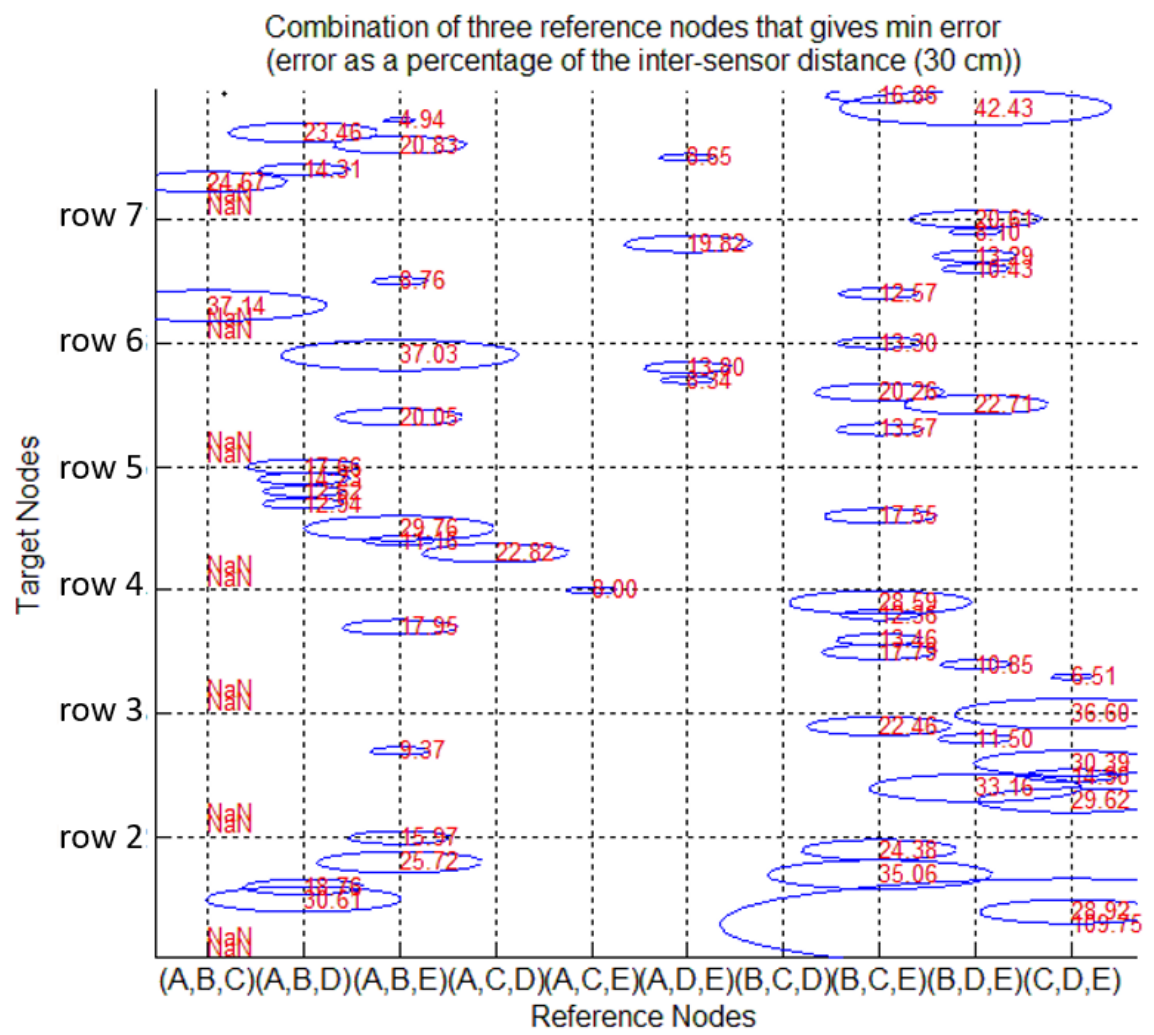

Figure 7.26. Reference node combinations with minimum target estimation error (Adjoining wall) 

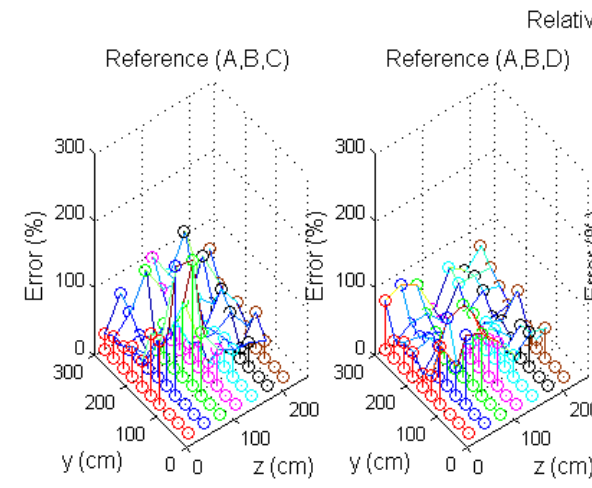

Relative Position Estimation Error from Reference Nodes (\%)
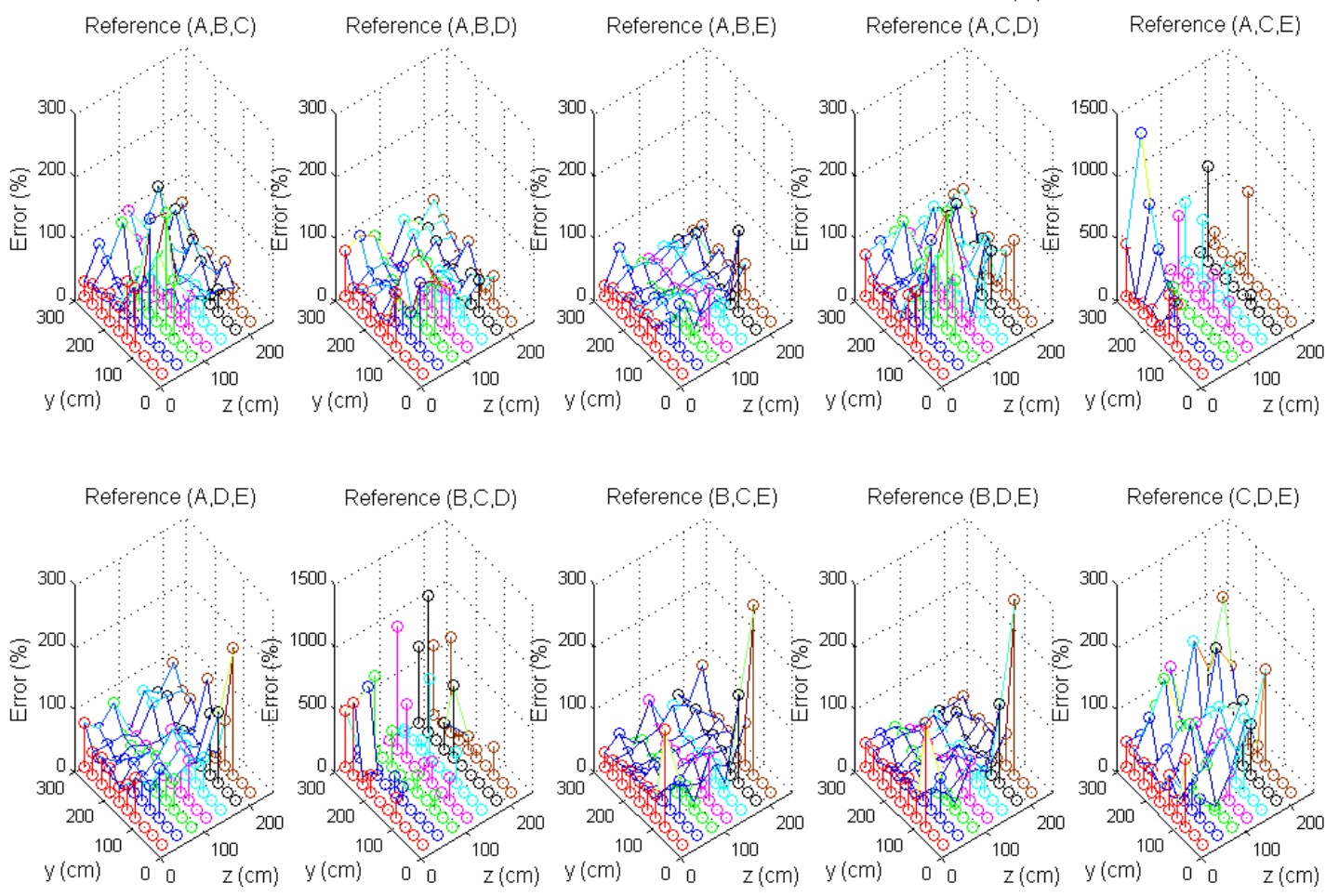

Figure 7.27. Normalized relative target position estimation error for adjoining wall (normalized to inter-target distance of $30 \mathrm{~cm}$ )

Figure 7.27 subplots show the relative target position estimation error from each reference nodes as normalized value of inter-target distance of $30 \mathrm{~cm}$. And, Figure 7.28 shows best position estimation case for each target node from all combinations of reference nodes, relative to inter-sensor distance of $30 \mathrm{~cm}$. Most cases are less than $20 \%$ which is about $6 \mathrm{~cm}$. 


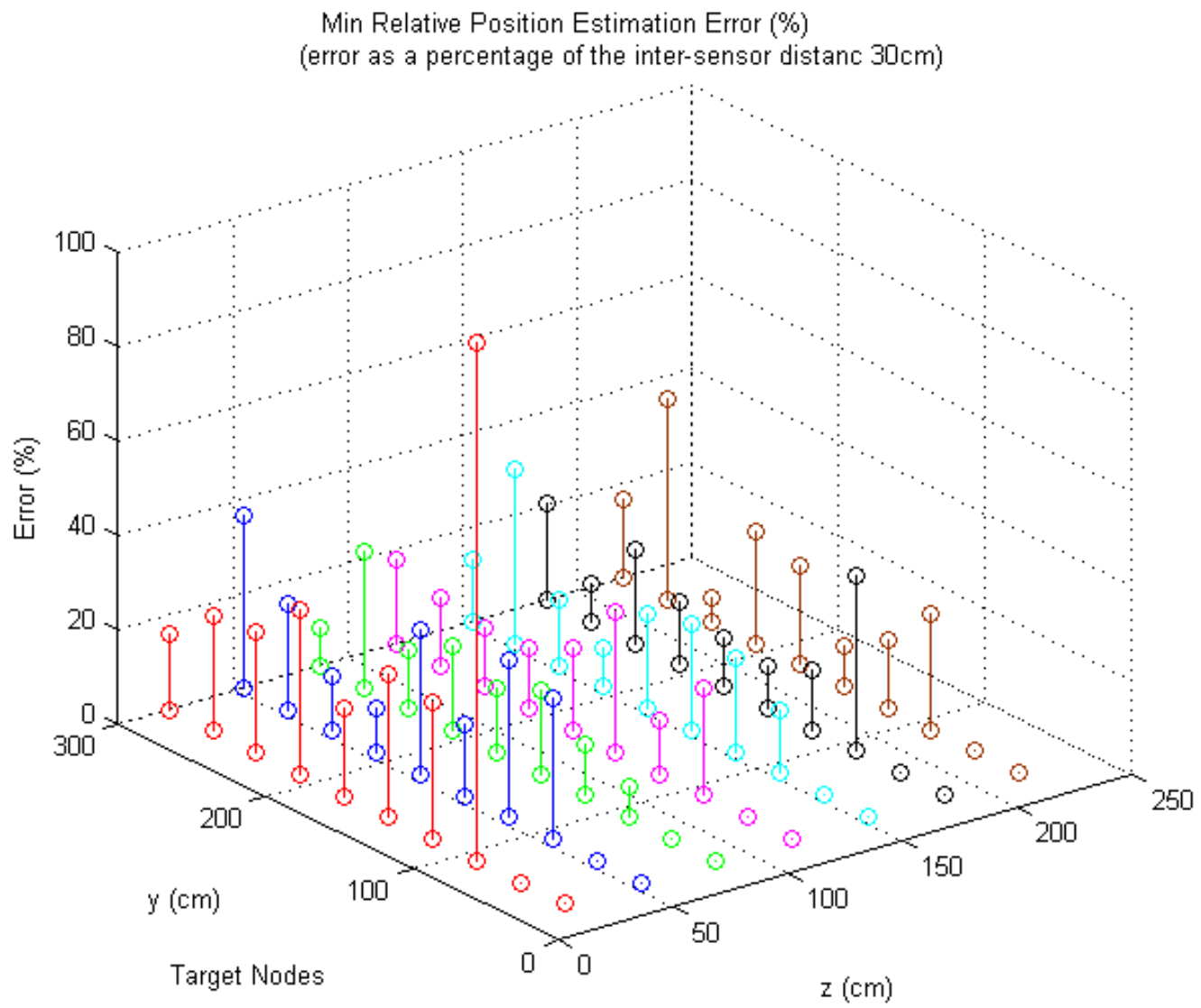

Figure 7.28. Minimum relative target position estimation error for adjoining wall 


\subsection{RESULTS FOR OPPOSITE WALL ESTIMATION}

This section explains the result of the target node (e.g. sensor 2) location estimation from the reference nodes (e.g. multiple sensor 1's) on the opposite wall placement. The method that estimates the position of the target node is the same as the one described in section 7.5 for the adjoining wall case, which uses distance measurement from the reference nodes.

As explained in section 7.5, we start by plotting all estimation results in Figure 7.29, and then excluding results with imaginary numbers (e.g. cyan and yellow lines) in Figure 7.30. After that, we remove results from reference nodes that form a straight line in Figure 7.31.

From the final set of results in Figure 7.31, the target position estimation error was calculated by calculating Euclidean distance between the actual target node position and the estimated position. Consecutively, the mean square error (MSE) of target position estimation error was calculated for each target node. Figure 7.32 shows the cumulative probability plot of the MSE distribution of target position estimation error. It can be seen that $90 \%$ of estimation error is less than $25 \mathrm{~cm}$. The mean MSE of target position estimation error was $16.68 \mathrm{~cm}$ (Table 7.6).

Table 7.6. Target node estimation result (opposite wall)

\begin{tabular}{|l|l|}
\hline Description (Opposite Wall) & Mean of MSE \\
\hline Location estimation error between actual tar- & $16.68(\mathrm{~cm})$ \\
get position and estimated positions & \\
\hline
\end{tabular}




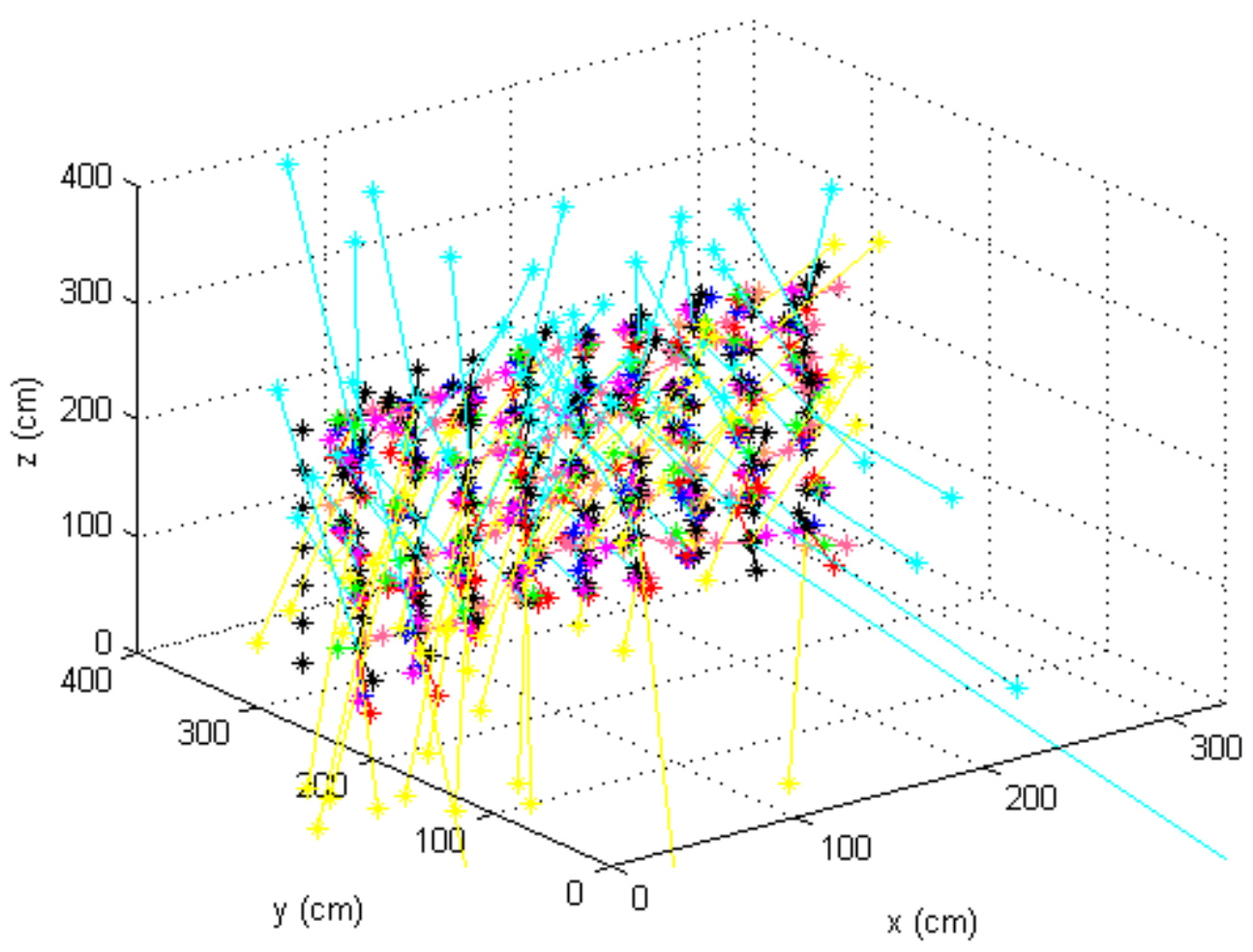

Figure 7.29. Estimated target positions with connected lines from actual target positions (Opposite wall) 


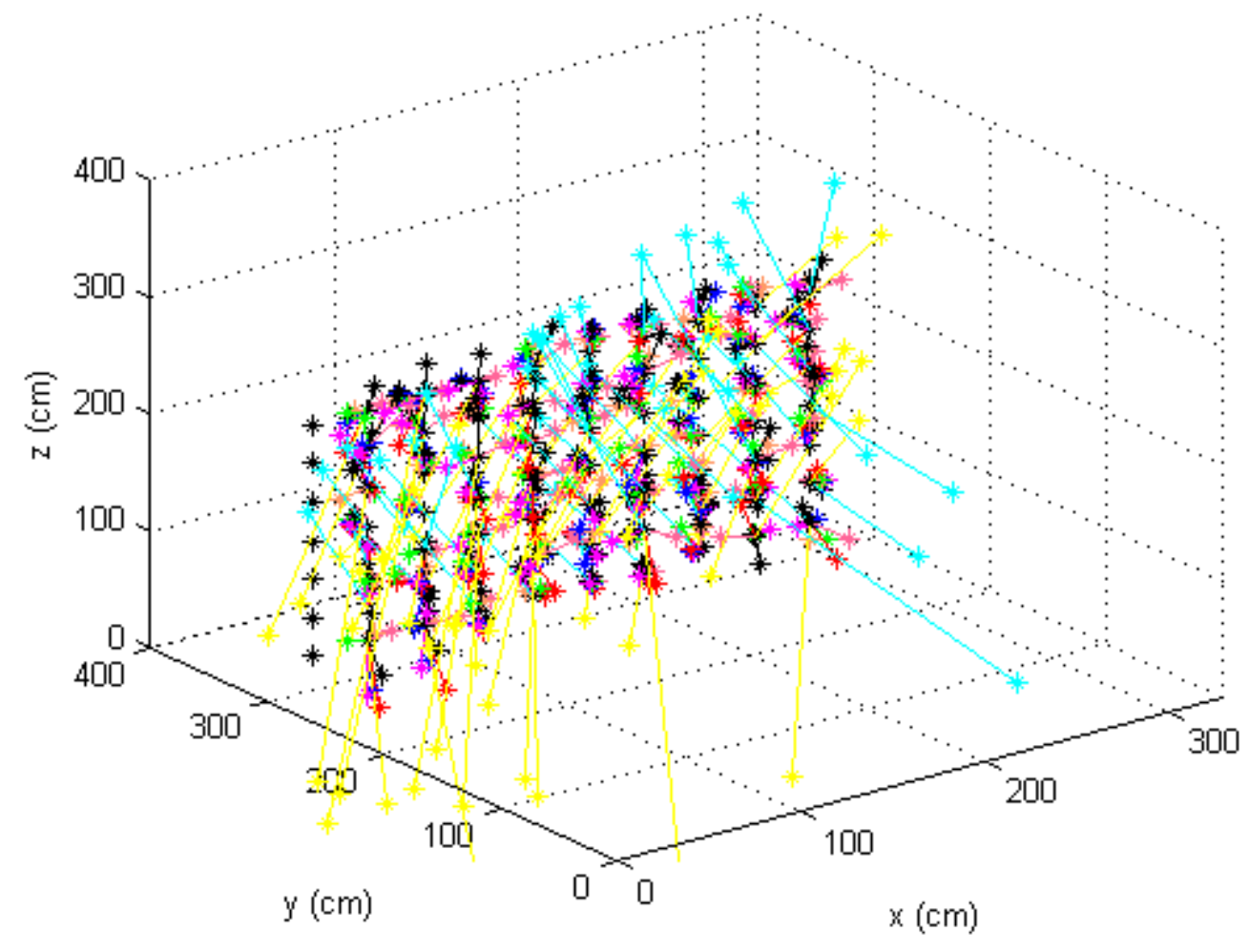

Figure 7.30. Estimated target positions with excluded estimations with imaginary numbers (Opposite wall) 


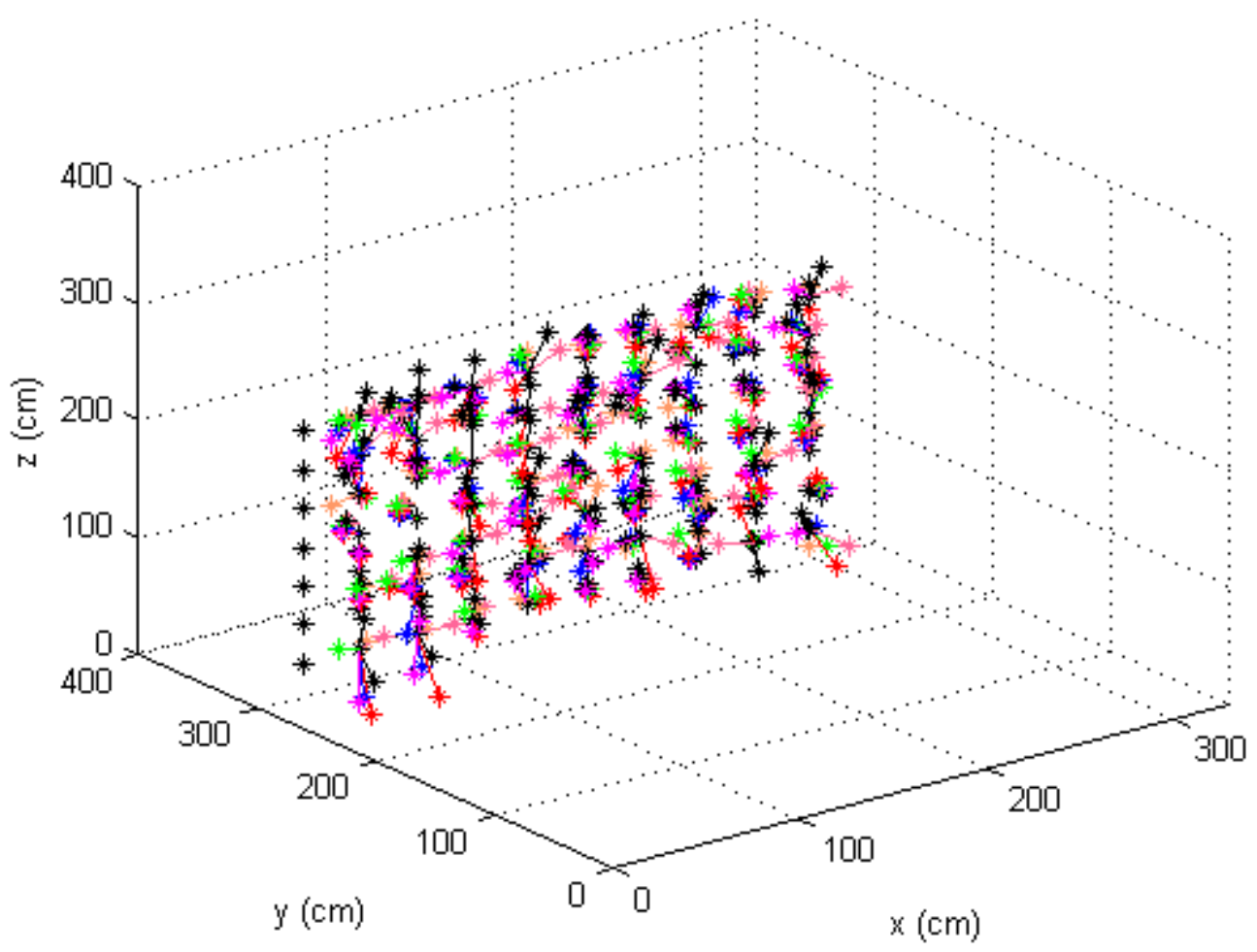

Figure 7.31. Estimated target positions with excluded estimations with imaginary numbers and from straight line reference nodes (Opposite wall) 


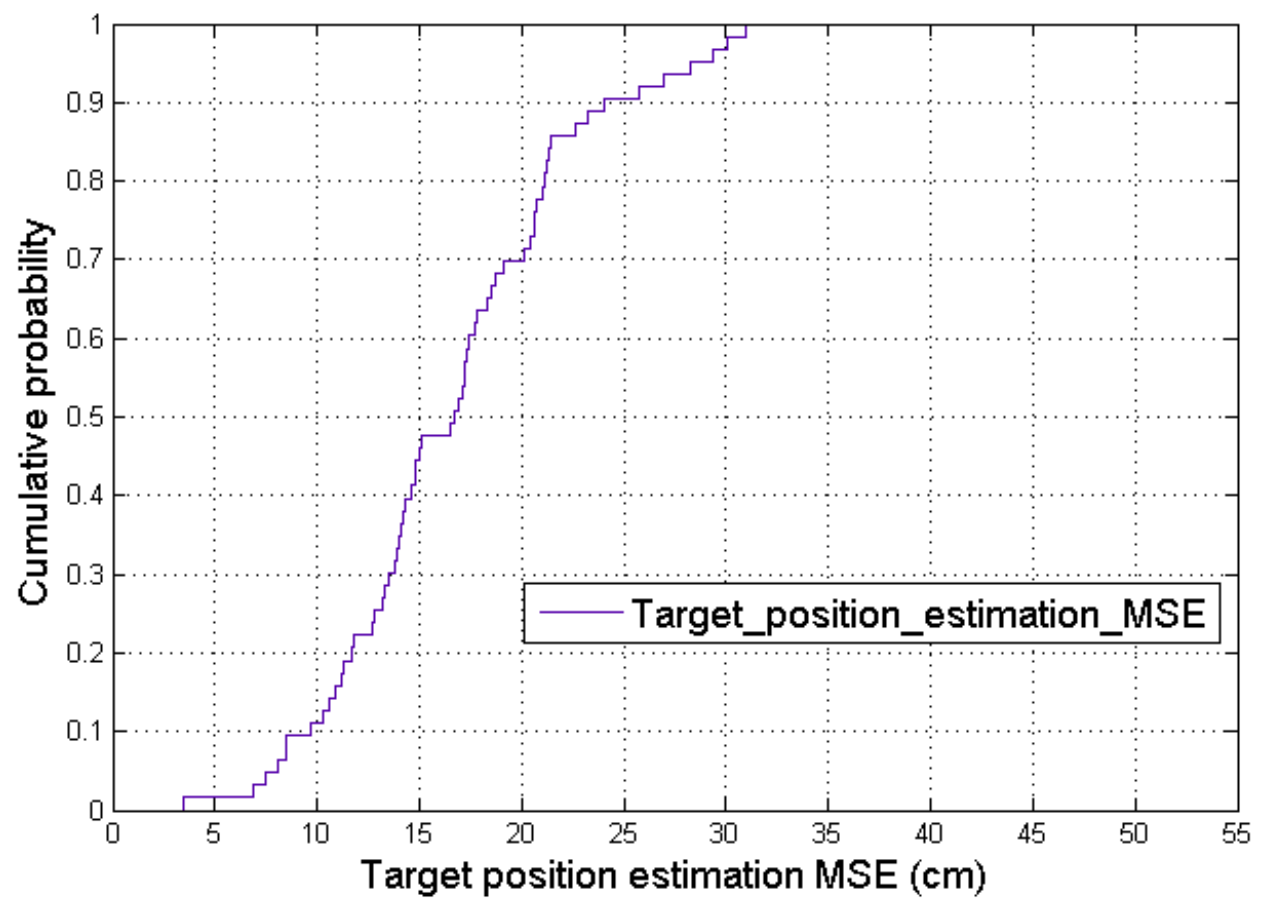

Figure 7.32. CDF of target node position estimation MSE (opposite wall) 
The combination of three reference nodes out of five (i.e. A to E) that gives minimum relative error for each target node is shown in Figure 7.33. It shows normalized error as a percentage of the inter-target node distance of $30 \mathrm{~cm}$. Most of errors were less than $30 \%$. It means that the best guesses had error of less than $9 \mathrm{~cm}$. Mean of all minimum errors was $6.6855 \mathrm{~cm}$. Below we summarize observations from Figure 7.33.

- In case of opposite wall placement, position estimation performance is similar across all combinations of reference nodes except for straight line cases. This is due to the fact that the distance between the reference node and target node is well separated at minimum 3 meters.

- Combinations such as (A, C, E) and (B, C, D) where reference nodes lie on a straight line don't perform well, similar to adjoining wall case.

- Overall, min location estimation error performance is similar to that of adjoining wall case.

Figure 7.34 subplots show relative target position estimation error from each reference nodes as normalized value of inter-target distance of $30 \mathrm{~cm}$. And, Figure 7.35 shows the best position estimation case for each target node from all combinations of reference nodes, relative to inter-target distance of $30 \mathrm{~cm}$. Most cases are less than $30 \%$ which is about $9 \mathrm{~cm}$.

\subsection{CONCLUSIONS FOR 3D WALL MAPPING}

In this chapter, we first presented the measurement error analysis of ToA based distance and AoA for both adjoining and opposite wall in terms of mean error and $95 \%$ error range. The $95 \%$ error of distance measurement was in the range 


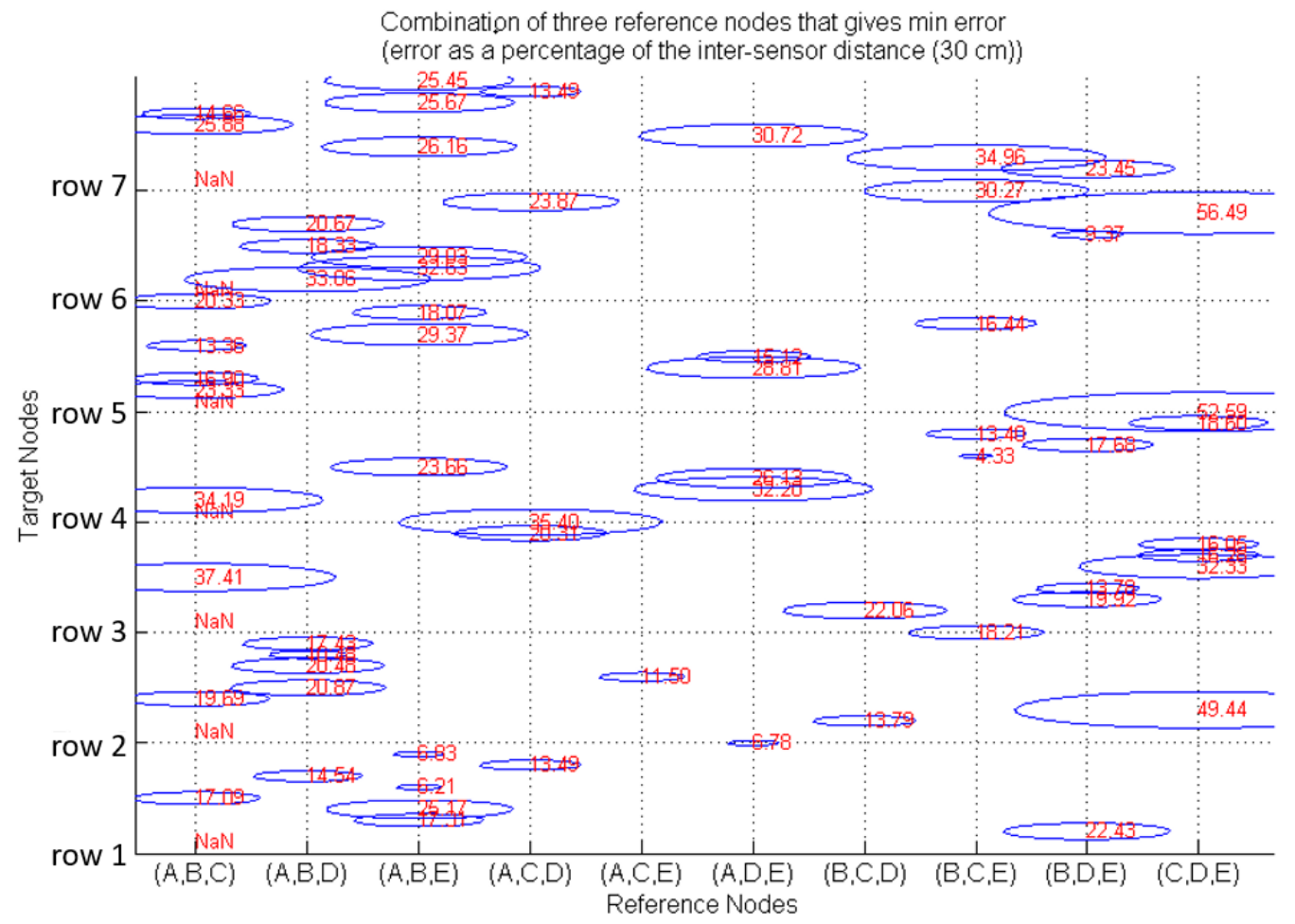

Figure 7.33. Reference node combinations with minimum target estimation error (opposite wall) 

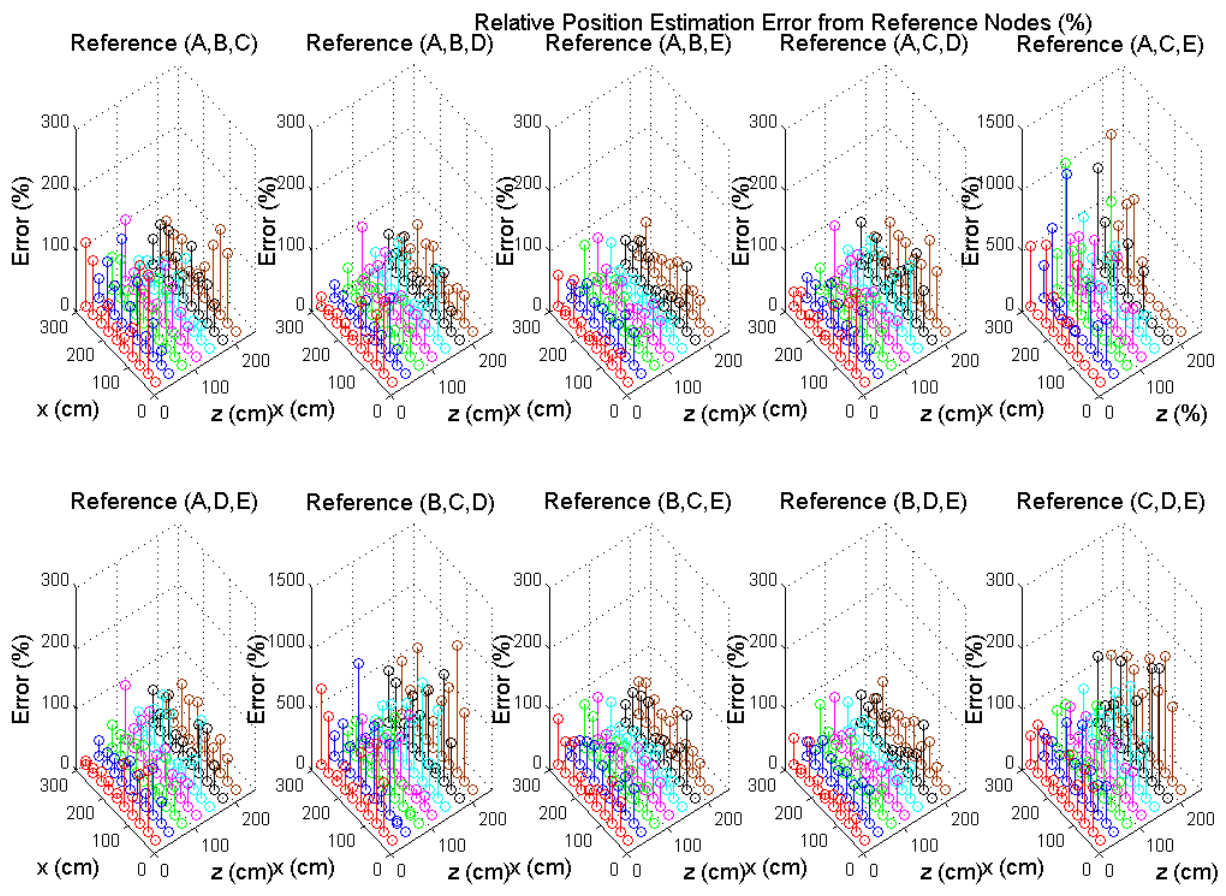

Figure 7.34. Normalized relative target position estimation error from each reference node combination (opposite wall) 


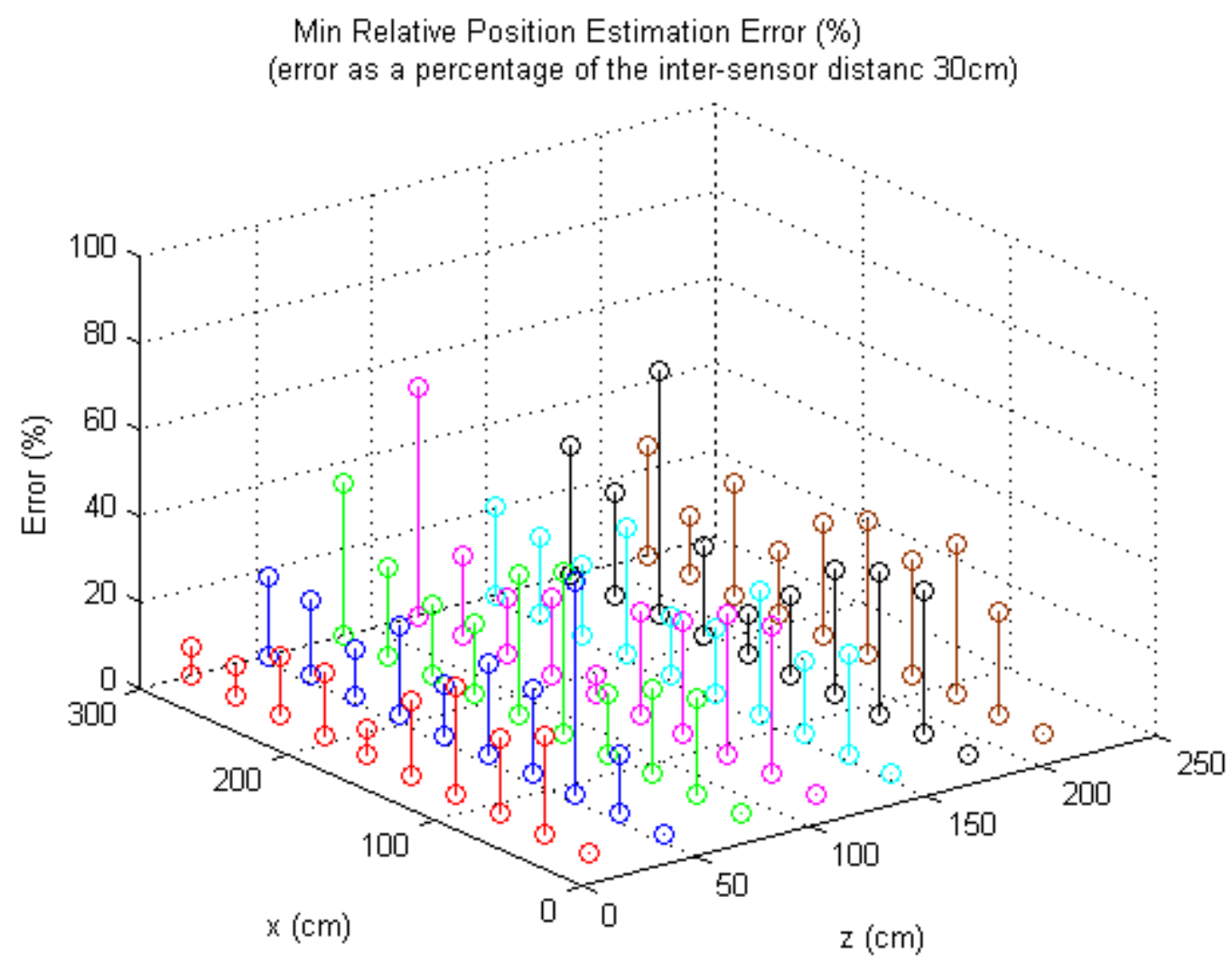

Figure 7.35. Minimum relative target position estimation error (opposite wall) 
of $0.00 \%$ and $15.89 \%$, which is less than $5 \mathrm{~cm}$ error. The $95 \%$ error range of AoA measurement for both adjoining and opposite wall was between $0.17 \%$ and $393.33 \%$. Based on this distance measurement, the positions of target nodes on adjoining wall $\mathrm{B}$ and opposite wall $\mathrm{C}$ were estimated and the results were presented. The mean of MSE of target node estimation was $16.73 \mathrm{~cm}$ for adjoining wall and $16.68 \mathrm{~cm}$ for opposite wall. The accurate distance measurement using UWB radios provided good position estimation of target nodes. When plotted estimated target node positions, the shape of adjoining or opposite wall could be reconstructed in reference to the reference nodes. 
Chapter 8

RELATED WORK

Our thesis has two primary areas of study: material characterization using radio and indoor wall mapping. In this chapter, we present related research in these areas.

\subsection{MATERIAL CHARACTERIZATION}

Technologies other than UWB such as X-ray tomographic imaging [33] and Ground Penetrating Radar (GPR) [49][62][67][81][94][9] are being applied to determine concrete thickness, to create an image of the concrete interior structure, to detect fractures and voids, and for other applications such as human respiration monitoring [58]. GPR gained its popularity over X-ray tomographic imaging due to its low cost operation and safeness. However, not much work has been done that determines wall thickness using UWB technology, which is critical in locating transceivers precisely and in creating the image of building or rubble structure. GPR operates in the microwave band of $300 \mathrm{MHz}$ up to $3 \mathrm{GHz}$ which is lower than UWB spectrum of $3.1 \mathrm{GHz}$ to $10.6 \mathrm{GHz}$. Thus, estimation using UWB gives less error than using GPR, which is critical in locating transceivers precisely and in creating the image of building or rubble structure. One other major difference is that GPR uses signal reflection technique, while our methodology uses signal transmission technique. 
Material sensing techniques using GPR are largely based on characterizing reflection coefficient of reflected signal. [106] presents GPR technique that simultaneously determines the number of layers, thickness, and the dielectric constants of multiple layers of material. The measurement setup uses a network analyzer sweeping over the frequency range of $30 \mathrm{kHz}$ to $3 \mathrm{GHz}$ to simulate a short pulse generation and to estimate the reflection coefficient of the material from the measured reflected signal. An inverse scattering algorithm was used to get thickness and the dielectric constants. [76] also studies the dielectric characterization of reflection coefficients of material. It estimates complex permittivity and multipath inside material using the algorithm based on the matrix pencil method. The measurement setup with vector network analyzer was used in an anechoic chamber in $8 \mathrm{GHz}$ to $12.5 \mathrm{GHz}$ band. [88] extends the dielectric characterization problem to the case of inhomogeneous materials: either it consists of multiple layers of different materials or materials that exist only in a form of a mixture of multiple components (i.e., materials with air void or a meat with fat).

Dielectric constant characterization with UWB radio has been studied in [75]. Different from GPR which exploits reflected signals [76][88][103], it uses transmission technique to characterize the dielectric constants of common building materials. [54] also characterizes the dielectric constant of building materials using UWB radios in $3 \mathrm{GHz}$ to $10 \mathrm{GHz}$ bands, and [21] in $5.8 \mathrm{GHz}$ band. The building materials used in [54] (e.g. Ca-Si board, Chipwood, Glass, Plasterboard) and [21] (e.g. Glass, Chipwood, Plasterboard, Brick wall) are different from the materials that our research used (e.g. concrete, reinforced concrete, drywall, plywood). [26] models propagation in and around homes and trees in $5.8 \mathrm{GHz}$ band as well. Their propagation study is for outdoor path loss or house level penetration loss 
using three houses, whereas our study is for indoor path loss and material level penetration study.

\subsection{INDOOR POSITIONING}

In recent years the problem of locating sensors in a sensor network or mobile devices in WLAN networks has received a great deal of attention as the Federal Communications Commission (FCC) required the precise location of emergency 911 callers. Outdoor/Indoor positioning have been found to be very useful not only for public safety but also for business creation such as targeted advertising, location sensitive billing, asset tracking [78][83], etc.

Standardization bodies such as $3 \mathrm{GPP}$ responded to the order from the FCC [19] on Wireless E911 Location Accuracy Requirements and studied the indoor positioning enhancement methods in Rel-13 TR37.857 [1]. It took two main approaches, one being Radio Access Technology (RAT) dependent method and the other being RAT-independent. RAT-dependent techniques include OTDOA, ECID, etc., and RAT-independent techniques studies terrestrial beacon system, collaborative positioning with $\mathrm{Wi}-\mathrm{Fi} / \mathrm{BT} /$ Sensors, etc.

Techniques developed for indoor positioning are different from the one for outdoor positioning which uses satellite/GPS because GPS signal is absent indoor and they face unique challenges. The problem is to find the coordinate of the target node. The position of the target node can be identified by obtaining position related signal parameters from one or more reference nodes and then using geometric relationships of those parameters in data fusion step to estimate the position. The data fusion step combines obtained signal parameters such as received signal strength (RSS), time of arrival (ToA), Time Difference of Arrival (TDoA), 
and angle of arrival (AoA) and applies geometric techniques such as trilateration, triangulation, and Hybrid method to determine the position.

\subsubsection{RSS based Indoor Positioning}

RSS method employs the pathloss model translating the difference between the transmitted signal strength and the received signal strength into a range estimate [60]. The challenge in using RSS is that due to the multipath fading and shadowing in the indoor environment the pathloss model does not hold, thus making reliable distance estimation indoors virtually impossible. However, several approaches, called variously fingerprinting or radio maps, have yielded reasonably good results by utilizing the pre-generated RSS database over a dense grid of positions throughout the floor plan of the building [7][39][40][50][51][109][87]. RSS values from a node are compared against the database and their location identified. Despite the various problems with this approach due to interference with other mobiles or propagation environment change due to furniture, the systems demonstrated accuracy of the order of several meters with the error of the order of a couple of meters.

\subsubsection{ToA based Indoor Positioning}

ToA approaches rely on the estimation of the flight time of a signal between a pair of nodes assuming a Line-of-Sight (LoS) propagation environment. The nodes should be tightly synchronized. A ToA measurement specifies the circle of the possible positions of the target node. The location of the target node can be determined with three measurements from reference nodes at the intersection of circles centered at each reference node. However, often indoor environments may not have a LoS propagation channel and the time of an arriving signal may suffer 
from multipath effects resulting in large error. There have been extensive studies and efforts to improve the ToA estimation precision that combats these drawbacks [22][23][2][37][101]. [38] studied TOA estimation algorithms for low sampling rate UWB systems by adaptively selecting threshold of minimum and maximum energy samples.

\subsubsection{TDoA based Indoor Positioning}

TDoA only requires the reference nodes to be synchronized [11] and utilizes the time difference of two arriving signals between the target node to two reference nodes. Each TDoA measurement defines a hyperbola for a position of the target node and the target node is then assumed to be located at the overlap of multiple hyperbolae [34].

\subsubsection{AOA based Indoor Positioning}

AoA methods rely on the base station (BS) computing the angle of the mobile. Generally, the angle is found as a range so at each BS we get the mobile's position

estimated as a cone. The intersection of these cones gives us the location. 3-D AoA multipath propagation measurement and estimation in indoor environments including both the azimuth and elevation angles of multipath components was investigated across the UWB frequency range of 3.1 to $10.6 \mathrm{GHz}$ in [108]. However, its focus is accurate estimation of AoA parameter itself and not 3D locationing itself.

\subsubsection{RSSI based Indoor Positioning}

RSSI based method such as Wi-Fi fingerprint localization has attracted attention recently because it does not require LOS measurement and can benefit from 
pervasive wireless LAN (WLAN) deployment [41]. This localization scheme also first performs offline site survey to build database of the signal patterns followed by online signal measurement and its association with localization algorithm for location estimation [98][59][84][100].

\subsubsection{Hybrid Schemes for Indoor Positioning}

Finally, hybrid schemes that use more than one of these four methods have also been studied [13][20][72][93]. [40] deployed 3 APs on 3rd floor of a building tracking moving mobile station to estimate the impact of bandwidth to locationing.

\subsubsection{Challenges and Mitigation for Measurement Error}

Challenges such as multipath and NLOS result in significant measurement error and a great deal of work has been done to reduce this error. [61][38] uses beamforming technique to mitigate path overlapping effects in indoor positioning. [4][40] reduces the error with fingerprinting technique, by creating and using a database of estimated ToA measurements from many mobile terminals. Another technique [86] is to use directional transmit antennas. The mean error in distance estimate reduces by a factor of five as compared to omni-directional transmit antennas and the standard deviation of error reduces by a factor of 15 . The Best Linear Unbiased Estimate (BLUE) [45] uses a statistical model to position radios in $2 \mathrm{D}$ space. It tries to minimize the variance of the final estimation in the linear space. Expectation of $o(n)$ is in linear form of the true position $E(o(n))$.

Given the dependence of resolution on bandwidth [85], UWB radios appear to be

a good choice for accurate locationing. UWB transmitter localization effort using Time Difference of Arrival (TDOA) with multiple antenna pairs was conducted 
by Young, et al. [103], where the measurement was made in an anechoic chamber (W13 x D20 x H12m) placing trees or metallic objects in different positions of the anechoic chamber floor to create a multipath environment. The transceivers in this study had a LoS path, whereas signals in our study had non-line-of-sight (NLoS) harsher propagation conditions because of travel through building materials. [105] uses a moving transmitter and fixed receiver to produce a propagation map of a room.

Attempts to reduce the localization error in the NLoS case were made, e.g., [24][56][99], but excessive propagation delay in the NLoS condition was a limiting factor of precise ranging [56]. Wylie and Holtzman [99] used statistical information of the difference in the variance of time of arrival (TOA) in the line-of-sight (LoS) case and in the NLoS case. Algorithms that detect the first path under a multipath

condition were developed, e.g., [24][56]. Our algorithm targets locationing of a transmitter with very small error even with the considerable ranging error caused by excessive propagation delay in the NLoS case overcoming ranging limitation.

\subsection{UWB RADAR}

UWB technology has been used in UWB radar system that transmits signal in a much wider spectrum with very low power than conventional radar systems [97]. UWB radar usually transmits signal under thermal noise. The technique used to generate a UWB radar signal is to transmit pulses with very short time duration, e.g. less than 1 nanosecond. UWB radar uses signal reflection technology while IR-UWB technology uses signal transmission technology between the transceivers.

There were various studies on human detection system development and applications using UWB radar for military [42], medical [46], or emergency rescue 
[57] operations. Also UWB radar is useful in subsurface object detection such as landmines [73].

Time Domain Corporation [31] received a patent on wideband radar technology that detects the presence of an object or motion through a wall, which can be used in through wall sensing, rubble rescue, and others. Through-wall sensing of heart beat using Direction of Arrival (DOA) and reflection was proposed by Chia, et al. [16].

UWB life detection radar system prototype was built by [15] and the optimal bandwidth and center frequency of breathing motion detection UWB radar system was presented in [70][68][107][46], and [79] also studied human detection method using UWB radar. 


\section{Chapter 9}

\section{CONCLUSIONS}

In this thesis we consider the problem of estimating the wall type and wall mapping using transceiver-based UWB radios as sensors. The result shows good performance of our algorithm, and we can identify wall type and wall placement correctly with very low error.

The wall thickness and type of material estimation utilized signal propagation measurements using two UWB radios separated by some distance. Applying our algorithm shows accuracy of more than $98.7 \%$ in estimating material thickness and type. Also, the distance estimation error was less than $4.87 \%$ even under severe multipath indoor environments.

Our approach in estimating 3D wall mapping utilized 4 UWB radios for ToA based ranging and AoA measurement. We designed adjoining wall and opposite wall configuration and placed reference nodes and target nodes in different locations on the walls. Distance measurement mean error was less than $2.41 \%$ for all cases. The $95 \%$ error range of distance measurement was between $0 \%$ and $15.89 \%$, which is less than $5 \mathrm{~cm}$ error. Mean error for AoA measurement was less than 105.97 \%. High error happens when the actual angle being measured is close to 0 degrees. Even small absolute error is magnified when we consider relative error. 
Mean of MSE for location estimation error between actual target position and estimated position is less than $16.73 \mathrm{~cm}$ for both wall configurations. The accurate distance measurement using UWB radios provided good position estimation of target nodes. By plotting the estimated target node positions, the shape of adjoining wall or opposite wall could be reconstructed in reference to the reference nodes. 


\section{REFERENCES}

[1] 3GPP. Study on Indoor Positioning enhancements for UTRA and LTE (Release 13). https://portal.3gpp.org/desktopmodules/Specifications/ SpecificationDetails . aspx? specificationId=2629, 2015.

[2] Arash Abbasi and Mohammad Hossein Kahaei. Improving source localization in LOS and NLOS multipath environments for UWB signals. In Computer Conference, 2009. CSICC 2009. 14th International CSI, pages 310-316. IEEE, 2009.

[3] Akeem A Adebomehin and Stuart D Walker. Ultra-wideband signals for high-resolution cognitive positioning techniques in 5G wireless. In Sarnoff Symposium, 2016 IEEE 37th, pages 1-2. IEEE, 2016.

[4] Bardia Alavi and Kaveh Pahlavan. Bandwidth effect on distance error modeling for indoor geolocation. In Personal, Indoor and Mobile Radio Communications, 2003. PIMRC 2003. 14th IEEE Proceedings on, volume 3, pages 2198-2202. IEEE, 2003.

[5] Cesare Alippi, Giuseppe Anastasi, Mario Di Francesco, and Manuel Roveri. Energy management in wireless sensor networks with energy-hungry sensors. IEEE Instrumentation $\&$ Measurement Magazine, 12(2), 2009.

[6] Giuseppe Anastasi, Marco Conti, and Mario Di Francesco. Extending the lifetime of wireless sensor networks through adaptive sleep. IEEE Transactions on Industrial Informatics, 5(3):351-365, 2009. 
[7] Paramvir Bahl and Venkata N Padmanabhan. RADAR: An in-building RFbased user location and tracking system. In INFOCOM 2000. Nineteenth Annual Joint Conference of the IEEE Computer and Communications Societies. Proceedings. IEEE, volume 2, pages 775-784. Ieee, 2000.

[8] Paul Bedell. Wireless crash course. McGraw-Hill Professional, 2001.

[9] Andrea Benedetto. A three dimensional approach for tracking cracks in bridges using GPR. Journal of Applied Geophysics, 97:37-44, 2013.

[10] David Braginsky and Deborah Estrin. Rumor routing algorithm for sensor networks. In Proceedings of the 1st ACM international workshop on Wireless sensor networks and applications, pages 22-31. ACM, 2002.

[11] James J Caffery Jr. Wireless location in CDMA cellular radio systems, volume 535. Springer Science \& Business Media, 2006.

[12] Srdjan Čapkun, Maher Hamdi, and Jean-Pierre Hubaux. GPS-free positioning in mobile ad hoc networks. Cluster Computing, 5(2):157-167, 2002.

[13] Amer Catovic and Zafer Sahinoglu. The Cramer-Rao bounds of hybrid TOA/RSS and TDOA/RSS location estimation schemes. IEEE Communications Letters, 8(10):626-628, 2004.

[14] C-H Chang and Wanjinn Liao. Revisiting relative location estimation in wireless sensor networks. In Communications, 2009. ICC'09. IEEE International Conference on, pages 1-5. IEEE, 2009.

[15] Chao Chen, Jinjin Shao, Hejun Yin, Shengwei Meng, Jie Chen, and Guangyou Fang. Data acquisition system of ultra-wide band life detection 
radar based on PC104 and FPGA. In Systems and Informatics (ICSAI), 2012 International Conference on, pages 380-384. IEEE, 2012.

[16] MYW Chia, SW Leong, CK Sim, and KM Chan. Through-wall UWB radar operating within FCC's mask for sensing heart beat and breathing rate. In Radar Conference, 2005. EURAD 2005. European, pages 267-270. IEEE, 2005.

[17] Krishna Chintalapudi, Tat Fu, Jeongyeup Paek, Nupur Kothari, Sumit Rangwala, John Caffrey, Ramesh Govindan, Erik Johnson, and Sami Masri. Monitoring civil structures with a wireless sensor network. IEEE Internet Computing, 10(2):26-34, 2006.

[18] Federal Communications Commission. First report and order 0248. https://transition.fcc.gov/Bureaus/Engineering_Technology/ Orders/2002/fcc02048.pdf.

[19] Federal Communications Commission. Forth report and Order. https: //apps.fcc.gov/edocs_public/attachmatch/FCC-15-9A1.pdf, 2015.

[20] Li Cong and Weihua Zhuang. Hybrid TDOA/AOA mobile user location for wideband CDMA cellular systems. IEEE Transactions on Wireless Communications, 1(3):439-447, 2002.

[21] Inigo Cuinas and M García Sánchez. Building material characterization from complex transmissivity measurements at $5.8 \mathrm{GHz}$. IEEE Transactions on Antennas and Propagation, 48(8):1269-1271, 2000.

[22] Davide Dardari, Andrea Conti, Ulric Ferner, Andrea Giorgetti, and Moe Z Win. Ranging with ultrawide bandwidth signals in multipath environments. Proceedings of the IEEE, 97(2):404-426, 2009. 
[23] Davide Dardari, Andrea Giorgetti, and Moe Z Win. Time-of-arrival estimation of UWB signals in the presence of narrowband and wideband interference. In Ultra-Wideband, 200\%. ICUWB 200\%. IEEE International Conference on, pages 71-76. IEEE, 2007.

[24] B Denis, J Keignart, and N Daniele. Impact of NLOS propagation upon ranging precision in UWB systems. In Ultra Wideband Systems and Technologies, 2003 IEEE Conference on, pages 379-383. IEEE, 2003.

[25] Newport Beach Fire Department. Light Search and Rescue. http://www . nbcert.org/lightsearchrescue.htm.

[26] Greg Durgin, Theodore S Rappaport, and Hao Xu. Measurements and models for radio path loss and penetration loss in and around homes and trees at 5.85 GHz. IEEE Transactions on Communications, 46(11):1484-1496, 1998.

[27] Prabal Dutta, Mike Grimmer, Anish Arora, Steven Bibyk, and David Culler. Design of a wireless sensor network platform for detecting rare, random, and ephemeral events. In Proceedings of the 4th international symposium on Information processing in sensor networks, page 70. IEEE Press, 2005.

[28] Michael Edgar, Jonathan Aw, John Dudley, Roger Marley, Anthony Reid, Zane Smith, Adrian Taylor, and Ross McAree. Characterization of a UWB transceiver for mining applications. In 2012 Australian Mining Technology Conference, pages 125-140. Colourwise Digital, 2012.

[29] Jeremy Elson and Deborah Estrin. Time synchronization for wireless sensor networks. IEEE, 2001.

[30] Christian C Enz, Amre El-Hoiydi, J-D Decotignie, and Vincent Peiris. 
WiseNET: an ultralow-power wireless sensor network solution. Computer, $37(8): 62-70,2004$.

[31] Larry W Fullerton. Time domain radio transmission system, April 182006. US Patent 7,030,806.

[32] Saurabh Ganeriwal, Ram Kumar, and Mani B Srivastava. Timing-sync protocol for sensor networks. In Proceedings of the 1st international conference on Embedded networked sensor systems, pages 138-149. ACM, 2003.

[33] Edward J Garboczi. Three-dimensional mathematical analysis of particle shape using X-ray tomography and spherical harmonics: Application to aggregates used in concrete. Cement and concrete research, 32(10):1621-1638, 2002.

[34] Sinan Gezici. A survey on wireless position estimation. Wireless personal communications, 44(3):263-282, 2008.

[35] Sinan Gezici and H Vincent Poor. Position estimation via ultra-wide-band signals. Proceedings of the IEEE, 97(2):386-403, 2009.

[36] Michael A Goodrich and Alan C Schultz. Human-robot interaction: a survey. Foundations and trends in human-computer interaction, 1(3):203-275, 2007.

[37] I Guvenc and Zafer Sahinoglu. Multiscale energy products for TOA estimation in IR-UWB systems. In Global Telecommunications Conference, 2005. GLOBECOM'05. IEEE, volume 1, pages 5-pp. IEEE, 2005.

[38] Ismail Guvenc and Zafer Sahinoglu. Threshold-based TOA estimation for impulse radio UWB systems. In Ultra-Wideband, 2005. ICU 2005. 2005 IEEE International Conference on, pages 420-425. IEEE, 2005. 
[39] Youngjune Gwon, Ravi Jain, and Toshiro Kawahara. Robust indoor location estimation of stationary and mobile users. In INFOCOM 2004. Twentythird AnnualJoint Conference of the IEEE Computer and Communications Societies, volume 2, pages 1032-1043. IEEE, 2004.

[40] Ahmad Hatami and Kaveh Pahlavan. Performance comparison of RSS and TOA indoor geolocation based on UWB measurement of channel characteristics. In Personal, Indoor and Mobile Radio Communications, 2006 IEEE 17th International Symposium on, pages 1-6. IEEE, 2006.

[41] Suining He and S-H Gary Chan. Wi-Fi fingerprint-based indoor positioning: Recent advances and comparisons. IEEE Communications Surveys $\&$ Tutorials, 18(1):466-490, 2016.

[42] Yuan He, Pascal Aubry, Francois Le Chevalier, and Alexander Yarovoy. Decentralised tracking for human target in multistatic ultra-wideband radar. IET Radar, Sonar \& Navigation, 8(9):1215-1223, 2014.

[43] Sandra M Hedetniemi, Stephen T Hedetniemi, and Arthur L Liestman. A survey of gossiping and broadcasting in communication networks. Networks, 18(4):319-349, 1988.

[44] Wendi Rabiner Heinzelman, Joanna Kulik, and Hari Balakrishnan. Adaptive protocols for information dissemination in wireless sensor networks. In Proceedings of the 5th annual ACM/IEEE international conference on Mobile computing and networking, pages 174-185. ACM, 1999.

[45] Charles R Henderson. Best linear unbiased estimation and prediction under a selection model. Biometrics, pages 423-447, 1975. 
[46] Igor Immoreev and Teh-Ho Tao. UWB radar for patient monitoring. IEEE Aerospace and Electronic Systems Magazine, 23(11):11-18, 2008.

[47] DKL International Inc. The DKL LifeGuard. http://www.dklabs.com/ images/LifeGuard1.pdf.

[48] Dytran Instruments, Inc. Single axis and triaxial DC MEMS style accelerometers. http://www.dytran.com/assets/PDF/SensorSelectorGuide.pdf.

[49] Erik M Johansson and Jeffrey E Mast. Three-dimensional ground penetrating radar imaging using synthetic aperture time-domain focusing. In Proc. SPIE, volume 2275, pages 205-214, 1994.

[50] Kamol Kaemarungsi and Prashant Krishnamurthy. Modeling of indoor positioning systems based on location fingerprinting. In INFOCOM 2004. Twenty-third AnnualJoint Conference of the IEEE Computer and Communications Societies, volume 2, pages 1012-1022. IEEE, 2004.

[51] Parameshwaran Krishnan, AS Krishnakumar, Wen-Hua Ju, Colin Mallows, and SN Gamt. A system for LEASE: Location estimation assisted by stationary emitters for indoor RF wireless networks. In INFOCOM 2004. Twentythird AnnualJoint Conference of the IEEE Computer and Communications Societies, volume 2, pages 1001-1011. IEEE, 2004.

[52] Joanna Kulik, Wendi Heinzelman, and Hari Balakrishnan. Negotiation-based protocols for disseminating information in wireless sensor networks. Wireless networks, 8(2/3):169-185, 2002.

[53] Oak Ridge National Laboratory. Wavelet-Based Heartbeat Detector. https://www.ornl.gov/sites/default/files/ORNL\%20Review\% 20v32n1\%201999.pdf. 
[54] Ray-Rong Lao, Jenn-Hwan Tarng, and Chiuder Hsiao. Transmission coefficients measurement of building materials for UWB systems in 3-10 GHz. In Vehicular Technology Conference, 2003. VTC 2003-Spring. The 57th IEEE Semiannual, volume 1, pages 11-14. IEEE, 2003.

[55] Jeong Eun Lee. RescueNet. http://www.rescuenet.cs.pdx.edu/, 2007.

[56] Joon-Yong Lee and Robert A Scholtz. Ranging in a dense multipath environment using an UWB radio link. IEEE Journal on Selected Areas in Communications, 20(9):1677-1683, 2002.

[57] Jing Li, Lanbo Liu, Zhaofa Zeng, and Fengshan Liu. Advanced signal processing for vital sign extraction with applications in UWB radar detection of trapped victims in complex environments. IEEE journal of selected topics in applied earth observations and remote sensing, 7(3):783-791, 2014.

[58] Wenzhe Li, Xijing Jing, Zhao Li, and Jianqi Wang. A new algorithm for through wall human respiration monioring using GPR. In Ground Penetrating Radar (GPR), 2012 14th International Conference on, pages 947-952. IEEE, 2012.

[59] Hongbo Liu, Yu Gan, Jie Yang, Simon Sidhom, Yan Wang, Yingying Chen, and Fan Ye. Push the limit of WiFi based localization for smartphones. In Proceedings of the 18th annual international conference on Mobile computing and networking, pages 305-316. ACM, 2012.

[60] Hui Liu, Houshang Darabi, Pat Banerjee, and Jing Liu. Survey of wireless indoor positioning techniques and systems. IEEE Transactions on Systems, Man, and Cybernetics, Part C (Applications and Reviews), 37(6):1067-1080, 2007. 
[61] Yanjia Luo and Choi Look Law. Indoor positioning using UWB-IR signals in the presence of dense multipath with path overlapping. IEEE Transactions on wireless communications, 11(10):3734-3743, 2012.

[62] Christiane Maierhofer. Nondestructive evaluation of concrete infrastructure with ground penetrating radar. Journal of Materials in Civil Engineering, 15(3):287-297, 2003.

[63] Dimitris E Manolakis. Efficient solution and performance analysis of 3-D position estimation by trilateration. IEEE Transactions on Aerospace and Electronic systems, 32(4):1239-1248, 1996.

[64] Usman Mehmood, Usman Mansoor, Dong Yeop Hwang, Ki-Hyung Kim, Taekkyeun Lee, and Seung Wha Yoo. Wireless Sensor Networks for integrated search and rescue efforts for disaster hit areas. In Ubiquitous and Future Networks (ICUFN), 2012 Fourth International Conference on, pages 306-309. IEEE, 2012.

[65] I Skolnik Merrill et al. Introduction to radar systems. Mc Grow-Hill, 2001.

[66] Robin R Murphy. Human-robot interaction in rescue robotics. IEEE Transactions on Systems, Man, and Cybernetics, Part C (Applications and Reviews), 34(2):138-153, 2004.

[67] Jindrich Musil and Frantisek Zacek. Microwave measurements of complex permittivity by free space methods and their applications. NASA STI/Recon Technical Report A, 87, 1986.

[68] Soumya Nag, Mark A Barnes, Tim Payment, and Gary W Holladay. An ultra-wideband through-wall radar for detecting the motion of people in real time. In Proceedings of SPIE, volume 4744, pages 48-57, 2002. 
[69] Georgia Tech Research News. Radar Vital Signs Monitor (RVSM). http: //gtresearchnews.gatech.edu/newsrelease/FLASH_SP.html.

[70] Amer Nezirovic, Alexander G Yarovoy, and Leo P Ligthart. Experimental verification of human being detection dependency on operational UWB frequency band. In Ultra-Wideband, 200\%. ICUWB 200\%. IEEE International Conference on, pages 305-310. IEEE, 2007.

[71] Yoshifumi Nishida, Hiroshi Aizawa, Toshio Hori, Nell H Hoffman, Takeo Kanade, and Masayoshi Kakikura. 3D ultrasonic tagging system for observing human activity. In Intelligent Robots and Systems, 2003.(IROS 2003). Proceedings. 2003 IEEE/RSJ International Conference on, volume 1, pages 785-791. IEEE, 2003.

[72] Kaveh Pahlavan, Xinrong Li, and Juha-Pekka Makela. Indoor geolocation science and technology. IEEE Communications Magazine, 40(2):112-118, 2002.

[73] G Pochanin, S Masalov, I Pochanina, L Capineri, P Falorni, and T Bechtel. Modern trends in development and application of the UWB radar systems. In Ultrawideband and Ultrashort Impulse Signals (UWBUSIS), 2016 8th International Conference on, pages 7-11. IEEE, 2016.

[74] Michael Robinson and Ioannis Psaromiligkos. Received signal strength based location estimation of a wireless LAN client. In Wireless Communications and Networking Conference, 2005 IEEE, volume 4, pages 2350-2354. IEEE, 2005. 
[75] Ahmad Safaai-Jazi, Sedki M Riad, Ali Muqaibel, and Ahmet Bayram. Ultrawideband propagation measurements and channel modeling. Report on Through-the-Wall Propagation and Material Characterization, 2002.

[76] Florence Sagnard and G El Zein. In situ characterization of building materials for propagation modeling: Frequency and time responses. IEEE Transactions on Antennas and Propagation, 53(10):3166-3173, 2005.

[77] Z Sahinoglu, S Gezici, and I Guvenc. Ultra-wideband positioning systems: Theoretical limits. Ranging Algorithms, and Protocols, 2008.

[78] Ali H Sayed, Alireza Tarighat, and Nima Khajehnouri. Network-based wireless location: challenges faced in developing techniques for accurate wireless location information. IEEE signal processing magazine, 22(4):24-40, 2005.

[79] Bernd Schleicher, Ismail Nasr, Andreas Trasser, and Hermann Schumacher. IR-UWB radar demonstrator for ultra-fine movement detection and vitalsign monitoring. IEEE transactions on microwave theory and techniques, 61(5):2076-2085, 2013.

[80] Rahul C Shah and Jan M Rabaey. Energy aware routing for low energy ad hoc sensor networks. In Wireless Communications and Networking Conference, 2002. WCNC2002. 2002 IEEE, volume 1, pages 350-355. IEEE, 2002.

[81] MR Shaw. The permittivity and conductivity of concretes at groundpenetrating radar frequencies. Advances in cement Research, 10(4):187-194, 1998.

[82] STMicroelectronics. Accelerometers. http://www.st.com/en/ mems-and-sensors/accelerometers . html?querycriteria=product Id= SC444. 
[83] Guolin Sun, Jie Chen, Wei Guo, and KJ Ray Liu. Signal processing techniques in network-aided positioning: a survey of state-of-the-art positioning designs. IEEE Signal Processing Magazine, 22(4):12-23, 2005.

[84] Wei Sun, Junliang Liu, Chenshu Wu, Zheng Yang, Xinglin Zhang, and Yunhao Liu. MoLoc: On distinguishing fingerprint twins. In Distributed Computing Systems (ICDCS), 2013 IEEE 33rd International Conference on, pages 226-235. IEEE, 2013.

[85] Z Tarique, WQ Malik, and DJ Edwards. Bandwidth requirements for accurate detection of direct path in multipath environment. Electronics Letters, 42(2):100-102, 2006.

[86] Zunnoor Tarique, Wasim Q Malik, and David J Edwards. Effect of bandwidth and antenna directivity on the range estimation accuracy in a multipath environment. In Vehicular Technology Conference, 2006. VTC 2006Spring. IEEE 63rd, volume 6, pages 2887-2890. IEEE, 2006.

[87] Andreas Teuber, Bernd Eissfeller, and Thomas Pany. A two-stage fuzzy logic approach for wireless LAN indoor positioning. In Proc. IEEE/ION Position Location Navigat. Symp, volume 4, pages 730-738, 2006.

[88] Kailash P Thakur and Allan G Williamson. Multiple dielectric slabs in waveguide cell. IEEE microwave and wireless components letters, 11(3):121$123,2001$.

[89] Timedomain. AoA Application Note Rev B. http://www.timedomain.com, 2003.

[90] Timedomain. EVK User's manual Rev D. http://www.timedomain.com, 2004. 
[91] Timedomain. 320-0094B P210 Getting Started Guide. http://www . timedomain. com, 2005.

[92] Timedomain. 320-0096E Fused Sample Application Note. http://www . timedomain.com, 2006.

[93] Lei Wang and Wai-Choong Wong. Fusion of multiple positioning algorithms. In Information, Communications and Signal Processing (ICICS) 2011 8th International Conference on, pages 1-5. IEEE, 2011.

[94] John P Warhus, Jeffery E Mast, Erik M Johansson, Scott E Nelson, and Hua Lee. Advanced ground-penetrating, imaging radar for bridge inspection. Technical report, Lawrence Livermore National Lab., CA (United States), 1993.

[95] Wikipedia. Accelerometer. http://en.wikipedia.org/wiki/ Accelerometer.

[96] Wikipedia. Ultra-wideband. http://en.wikipedia.org/wiki/ Ultra-wideband.

[97] Christian Wolff. What is an UWB radar. http://www.radartutorial.eu/ 02. basics/UWB\%20Radar . en . html.

[98] Chenshu Wu, Zheng Yang, Yunhao Liu, and Wei Xi. WILL: Wireless indoor localization without site survey. IEEE Transactions on Parallel and Distributed Systems, 24(4):839-848, 2013.

[99] Marilynn P Wylie and Jack Holtzman. The non-line of sight problem in mobile location estimation. In Universal Personal Communications, 1996. 
Record., 1996 5th IEEE International Conference on, volume 2, pages 827831. IEEE, 1996.

[100] Zhuoling Xiao, Hongkai Wen, Andrew Markham, Niki Trigoni, Phil Blunsom, and Jeff Frolik. Non-line-of-sight identification and mitigation using received signal strength. IEEE Transactions on Wireless Communications, 14(3):1689-1702, 2015.

[101] Allen Yi-Zhi Xu, Edward KS Au, Albert Kai-Sun Wong, and Qin Wang. A novel threshold-based coherent TOA estimation for IR-UWB systems. IEEE transactions on vehicular technology, 58(8):4675-4681, 2009.

[102] Jennifer Yick, Biswanath Mukherjee, and Dipak Ghosal. Wireless sensor network survey. Computer networks, 52(12):2292-2330, 2008.

[103] Derek P Young, Catherine M Keller, Dan W Bliss, and Keith W Forsythe. Ultra-wideband (UWB) transmitter location using time difference of arrival (TDOA) techniques. In Signals, Systems and Computers, 2004. Conference Record of the Thirty-Seventh Asilomar Conference on, volume 2, pages 12251229. IEEE, 2003.

[104] Yan Yu, Ramesh Govindan, and Deborah Estrin. Geographical and energy aware routing: A recursive data dissemination protocol for wireless sensor networks. 2001.

[105] Rudolf Zetik, Jürgen Sachs, and Reiner Thomä. Imaging of propagation environment by UWB channel sounding. COST273 Temporary Document TD (05), 58:1-11, 2005.

[106] Jietao Zhang, Mansor Nakhkash, and Yi Huang. Electromagnetic imaging of 
layered building materials. Measurement Science and Technology, 12(8):1147, 2001.

[107] Yang Zhang, Teng Jiao, Xijing Jing, Zhao Li, Sheng Li, Xiao Yu, Hao Lv, Zhu Zhang, and Jianqi Wang. A detecting and identifying method for two stationary human targets using single-channel ultra-wideband radar. EURASIP Journal on Advances in Signal Processing, 2012(1):202, 2012.

[108] Yongwei Zhang, Anthony K Brown, Wasim Q Malik, and David J Edwards. High resolution 3-D angle of arrival determination for indoor UWB multipath propagation. IEEE Transactions on Wireless Communications, 7(8), 2008.

[109] Junyang Zhou, KM-K Chu, and JK-Y Ng. Providing location services within a radio cellular network using ellipse propagation model. In Advanced Information Networking and Applications, 2005. AINA 2005. 19th International Conference on, volume 1, pages 559-564. IEEE, 2005. 